Aus dem Institut für Allgemeinmedizin

Institut der Ludwig-Maximilians-Universität München

Direktor: Prof. Dr. med. Jochen S. Gensichen

\title{
Medizinische Atteste und Untersuchungen in der allgemeinärztlichen Praxis
}

\section{Indikationen, Hindernisse und Relevanz einer Standardisierung}

\author{
Dissertation \\ zum Erwerb des Doktorgrades der Medizin \\ an der Medizinischen Fakultät der \\ Ludwig-Maximilians-Universität zu München
}

vorgelegt von

Livia Anna Elisabeth Avdotja Ertl

aus München

2019 
Mit Genehmigung der Medizinischen Fakultät der Universität München

Berichterstatter: Prof. Dr. med. Jörg Schelling

Mitberichterstatterin: PD Dr. med. Sandra Reilich

Betreuung durch die promovierte Mitarbeiterin: Dr. rer. nat. Linda Sanftenberg

Dekan: Prof. Dr. med. dent. Reinhard Hickel

Tag der mündlichen Prüfung: 28.02.2019 
Für Gicks 
Vorbemerkung

Alle männlichen Personen- und Funktionsbezeichnungen in dieser Arbeit beziehen sich in gleicher Weise auf Frauen und Männer. 


\section{Inhaltsverzeichnis}

$\begin{array}{ll}\text { 1. Einleitung } & 6\end{array}$

1.1. Gesundheitspolitische Debatte 6

1.2. Gesundheitsökonomische Aspekte 9

1.3. Internationaler Vergleich 10

1.4. Versorgungsqualität 12

2. Ziele der Untersuchung 15

$\begin{array}{ll}\text { 3. Methoden } & 16\end{array}$

3.1. Ethikvotum \& Datenschutz 16

3.2. Ablauf der Umfrage 17

3.3. Datenerhebung 20

3.4. Auswertung \& Statistik 22

4. Ergebnisse 26

4.1. Fragebogen 26

4.2. Gespräch 36

4.3. Attestbeispiele 46

$\begin{array}{ll}\text { 5. Diskussion } & 48\end{array}$

5.1. Interpretation der Ergebnisse 48

5.2. Kritische Betrachtung der Datenerhebung und Auswertung 64

6. Zusammenfassung 69

$\begin{array}{ll}\text { 7. Literaturverzeichnis } & 71\end{array}$

8. Anhang 77

8.1. Anschreiben, Probandeninformation und Fragebogen 77

8.2. Tabellarische Auflistung der Ergebnisse des Fragebogens 80

8.3. Gesprächsleitfaden $\quad 84$

8.4. Tabellarische Auflistung der Ergebnisse des Gesprächsleitfadens 88

8.5. Tabellarische Auflistung der Beispielatteste 90

8.6. Zusammenfassung der Ergebnisse und Handlungsempfehlungen 92

8.7. Ethikvotum 95

8.8. Danksagung 96

8.9. Eidesstattliche Versicherung 97 


\section{Einleitung}

Es gibt Diebe, die nicht bestraft werden und einem doch das Kostbarste stehlen: die Zeit. - Napoleon Bonaparte

Im Angesicht des kontinuierlich steigenden medizinischen Versorgungsbedarfs steigt der Wert der medizinischen Ressourcen ins Unermessliche. Die knapp bemessene Zeit der Ärzte sollte für Aufgaben reserviert sein, die ihrer Qualifikation entsprechen (Ertl L 2016). Deshalb gilt es, den zeitlichen Aufwand für nicht ärztliche Tätigkeiten zu minimieren und so den Zeitdieben das Handwerk zu legen.

\subsection{Gesundheitspolitische Debatte}

„Viel Zeit bliebe für die Behandlung der Patienten häufig nicht“ (Statistisches Bundesamt 2015, S.5). Im Angesicht des steigenden medizinischen Versorgungsbedarfs aufgrund der demographischen Entwicklung drängt sich die Frage auf, wie der bürokratische Aufwand zugunsten von mehr Behandlungszeit reduziert werden kann. Aus Sicht der Ärzte besteht hier dringender Handlungsbedarf (Statistisches Bundesamt 2015).

Der „Bürokratie Tsunami“ (Wenning 2010, S.1) ist ein hochaktuelles Thema der gesundheitspolitischen Debatte: Das Projekt Mehr Zeit für Behandlung, das die Bundesregierung 2013 im Auftrag gegeben hat, wurde 2015 abgeschlossen.

Die Kassenärztliche Bundesvereinigung (KBV) beschäftigt sich bereits seit 2006 mit dem Thema Entbürokratisierung und richtet gemeinsam mit der Kassenärztlichen Vereinigung Westfalen-Lippe (KVWL) das Best-Practice-Forum Bürokratieabbau aus, in dem Kassenärztlichen Vereinigungen verschiedener Regionen Erfahrungen zum Thema austauschen können.

Zusätzlich startete die KBV 2012 das Onlineforum Mehr Zeit für Patienten, das Ärzten ermöglicht Verbesserungsvorschläge einzureichen.

Die Kassenärztliche Vereinigung Bayerns (KVB) richtete 2011 die Anlaufstelle für Bürokratieabbau ein, um ebenfalls Anregungen aus der Praxis aufzugreifen und an die richtigen Stellen in Politik und Gesundheitswesen weiterzuleiten. 
Im Rahmen dieser Initiativen wurden erstmals Daten zum bürokratischen Aufwand erhoben. Als Teil der Informationspflichten wurde das Thema Atteste in der ambulanten Versorgung behandelt, stand aber nicht im Fokus der Untersuchungen (Ertl L 2016).

Die Dissertation soll einen ersten Überblick über die Rolle der Atteste und die dafür erforderlichen Untersuchungen im hausärztlichen Alltag liefern. In der vorliegenden Arbeit sollen Zeitaufwand und Abrechnungsmodalitäten der Attestausstellung in der Praxis eruiert werden. Aktuelle Vorgehensweisen, Schwierigkeiten und Verbesserungswünsche sollen herausgearbeitet werden, um das Potential einer Vereinheitlichung zu beleuchten (Ertl L 2016).

\section{Mehr Zeit für Behandlung}

Das Projekt Mehr Zeit für Behandlung - Vereinfachung von Verfahren und Prozessen in Arzt- und Zahnarztpraxen wurde vom Normenkontrollrat mit dem Statistischen Bundesamt und den Trägern der Selbstverwaltung im Gesundheitswesen durchgeführt. Das Ausmaß des bürokratischen Aufwands in Arztpraxen sollte erstmals quantifiziert werden, um Ursprünge besonders hoher Belastung zu identifizieren und unnötige Anforderungen abzubauen, wie das Statistische Bundesamt angibt.

Besonders die Summe und Häufigkeit vieler Informationspflichten wird als bürokratischer Aufwand wahrgenommen. Mit dem Standardkosten-Modell, das zur Berechnung der Bürokratiebelastung genutzt wurde, können Ausmaß und Verteilung von Bürokratie veranschaulicht werden. Die Untersuchung zeigt, dass in den Arzt- und Zahnarztpraxen Bürokratiekosten von über vier Milliarden Euro jährlich bestehen. Laut der Untersuchung ist jede Praxis im Schnitt rund 100 Arbeitstage pro Jahr nur mit der Erfüllung von Informationspflichten beschäftigt.

Die Zahlen zeigen, wie wichtig es ist, den zeitlichen Aufwand für bürokratische Tätigkeiten zu minimieren, um die Zeit für ärztliche Behandlung zu maximieren. Es liegt im dringenden Interesse der Gesundheitspolitik, die flächendeckende ambulante Versorgung auf diesem Weg sicherzustellen (Statistisches Bundesamt 2015). 


\section{Bessere Regulierung in der ambulanten Versorgung}

In Bessere Regulierung in der ambulanten Versorgung gelang der Kassenärztlichen Vereinigung Westfalen-Lippe mit dem Wirtschaftsprüfungsunternehmen KPMG eine erste Messung der bürokratischen Belastungen von Vertragsärzten und Psychotherapeuten in Westfalen-Lippe (KMPG 2006). Dabei wurden 281 Informationspflichten identifiziert, die allein in Westfalen-Lippe Kosten von 160 Millionen Euro im Jahr verursachen. Trotz unterschiedlicher Strukturverhältnisse der ambulanten Versorgung kann ein entsprechender Aufwand für Bayern angenommen werden. Für Gesamtdeutschland schätzte KPMG 2006 für den ambulanten Bereich Bürokratiekosten von ca. 1,6 Milliarden Euro.

\section{Anlaufstelle für Bürokratieabbau}

Die Anlaufstelle nahm allein im ersten Jahr 527 Meldungen aus bayerischen Arzt- und Psychotherapiepraxen entgegen. Die große Resonanz zu diesem Thema spricht für sich. Die meisten Meldungen betrafen Prozesse der Arznei-, Heil- und Hilfsmittelverordnung (12,5 \%), am zweithäufigsten wurden Anfragen der Krankenkassen (11,6 \%) und am dritthäufigsten die Praxisgebühr (11,4 \%) genannt.

Unter den Überbegriff Formulare entfielen insgesamt 11\% der Meldungen. Die Vordruckvereinbarung der Bundesmantelvertragspartner soll den Inhalt der Formulare regeln. Deshalb werden vor allem formfreie Anfragen, aber auch die Menge an verschiedenen, uneinheitlichen Formularen, als Quelle für Zeitaufwand, Verunsicherung im Bezug auf Datenschutz und erhöhte Fehlerrate beim Ausfüllen gesehen. Die Vorschläge bezogen sich unter anderem auf die Zusammenführung, Vereinheitlichung und Abschaffung von Formularen.

Nach Analyse der KVB konnten die meisten Meldungen, die nicht in den Zuständigkeitsbereich der Selbstverwaltung fallen, an die zuständigen Institutionen auf Bundesebene (z. B. Bundesministerium für Gesundheit, Partner der Bundesmantelverträge) oder Landesebene weitergeleitet werden (Kassenärztliche Vereinigung Bayerns 2014a). 


\subsection{Gesundheitsökonomische Aspekte}

Im Angesicht des steigenden Ärztemangels stellt sich die Frage, „wie lange es sich die Gesellschaft noch leisten will, kostbare ärztliche Arbeitskraft mit Verwaltungstätigkeiten zu verschwenden“ (Flintrop, Korzilius 2012, S.4).

Die Initiativen der letzten Jahre zeigen, wie wichtig die Reduktion des Verwaltungsaufwands ist, um mehr Zeit für die ärztliche Behandlung zu ermöglichen. Durch die konsekutive Reduktion der Verwaltungskosten könnte auch mehr Geld in die Behandlung des Patienten investiert werden.

Die Unternehmensberatung A.T. Kearney kalkulierte in der Untersuchung „Deutsches Gesundheitswesen auf dem Prüfstand - Kostenfalle Komplexität" ein Einsparpotential von 13 Milliarden Euro bei den Verwaltungskosten der Gesetzlichen Krankenversicherung. 41\% entfallen dabei auf den Bereich der niedergelassenen Ärzte.

Für das Jahr 2010 wurden Verwaltungskosten im Gesundheitswesen von 40,4 Milliarden Euro festgestellt. Daraus ergibt sich eine Verwaltungskostenquote von $23 \%$ im öffentlichen Gesundheitssystem, die um den Faktor 3,8 höher ist als der in der deutschen Industrie. Das bedeutet, dass von jedem Euro des Beitragszahlers höchstens 77 Cent für medizinische Leistungen übrig bleiben. Würde das von der Unternehmensberatung berechnete Einsparpotential genutzt, würden von jedem Euro 8 Cent mehr beim Patienten ankommen. Die Ergebnisse der Studie belegen ein großes Potential für Effizienzsteigerung durch eine Reduktion der Verwaltungskosten (A.T. Kearney, S.6). 


\subsection{Internationaler Vergleich}

Nach Einschätzungen der OECD (Organisation for Economic Co-operation and Development) und der Weltbank ist Deutschland eines der Länder mit der höchsten Bürokratiebelastung. Auch das Gesundheitswesen leidet unter der starken Regulierung (Müller et al 2006). In Deutschland wird die ambulante Versorgung häufiger in Anspruch genommen als in anderen Ländern. Die Regelungen zur Arbeitsunfähigkeitsbescheinigung $(A U)$ sind dafür ein wichtiger Einflussfaktor. Als Anlass für den Besuch beim Hausarzt geben vor allem Patienten mit leichteren Beschwerden an, dass sie eine Krankschreibung brauchen (Herrmann et al 2015).

Reformen der Regelungen, bei denen die Arbeitsunfähigkeitsbescheinigung erst nach längerer Zeit eingereicht werden muss, konnten in anderen europäischen Ländern Erfolge verzeichnen (OECD 2010).

\section{Niederlande}

In den Niederlanden können Arbeitnehmer sich selbst bis zu zu sechs Wochen krankschreiben. Danach muss der Arbeitsmediziner des Unternehmens die Arbeitsbedingungen im Hinblick auf den Gesundheitszustand des Patienten begutachten und gemeinsam mit Arbeitgeber und -nehmer einen Plan für die Wiedereingliederung erarbeiten. Dabei werden Anpassungen der Arbeitszeiten, Rückenschulungen oder Verhaltenstherapien eingesetzt.

Die Unterstützung des Return-to-work-Prozesses zeigt klaren Erfolg: Innerhalb von drei Jahren sank die Gesamt-Arbeitsunfähigkeitsquote von 5,3 auf 4\%. Auch die Zugänge der Erwerbsunfähigkeitsrente verringerte sich von 1 auf 0,4\% (De Boer 2010).

\section{Schweden}

Auch in Schweden wird der Return-to-work-Prozess stark unterstützt. Dabei werden in der sogenannten Rehabilitationskette Arbeitgeber und -nehmer, Ärzte und Sozialversicherungen frühzeitig eingebunden, um eine Rückkehr an den Arbeitsplatz zu voranzutreiben. Zur Verbesserung der Ausstellung der Arbeitsunfähigkeitsbescheinigung 
wurden Fortbildungsprogramme angeboten und Leitlinien erarbeitet, die die Druchschnittsdauer für die wichtigsten Krankheiten festlegen sollen (Torges 2010). Seit der Reform in Schweden sank der Anteil der arbeitsunfähigen Erwerbspersonen von 4,2\% auf 2,2\% (Kühbauch, Weber 2010). Ärzte geben an, dass die Reformen die Bescheinigung der Arbeitsunfähigkeit erleichtert haben, auch wenn sie sie immer noch als Belastung sehen (Carlsson et al 2015).

\section{Großbritannien}

Um den Return-to-work-Prozess zu fördern, wurde in Großbritannien die Fit Note eingeführt. Das Pendant der Sick Note soll sich auf die erhalten Fähigkeiten des Patienten konzentrieren.

Auf der Fit Note kann der Arzt erklären, in welchem Maß der Gesundheitszustand des Patienten seine Leistung am Arbeitsplatz beeinflusst und unter welchen Bedingungen eine Wiedereingliederung möglich ist. Dabei kann der Arzt konkrete Vorschläge zu Arbeitszeiten und anderen Anpassungen machen. Wenn der Arzt aktuell keine Möglichkeit für eine Rückkehr sieht, kann er den Patienten an einen Arbeitsmediziner überweisen (Kühbauch 2010). Zwei Jahre nach Einführung der Fit Note konnte bereits ein deutlicher Rückgang der Krankschreibungen verzeichnet werden, Vorschläge zur Anpassung der Arbeitsbedingungen stiegen dafür (Hussey et al 2015).

Die erfolgreichen Reformen zeigen Handlungsspielraum auf dem Feld der Arbeitsunfähigkeitsregelungen, der auch in Deutschland genutzt werden sollte. 


\subsection{Versorgungsqualität}

\section{Einflussfaktoren}

Die „Gesamtheit von Eigenschaften und Merkmalen eines Produktes oder einer Dienstleistung, die es oder sie zu erfüllen hat" als Definition von Qualität wird im Gesundheitswesen als „ausreichende und zweckmäßige, an der Lebensqualität orientierte, fachlich qualifizierte, aber auch wirtschaftliche medizinische Versorgung mit dem Ziel die Wahrscheinlichkeit erwünschter Behandlungsergebnisse zu erhöhen“ konkretisiert (Birkner et al 2003, S.16).

Aus der Definition geht hervor, dass ein wirtschaftlicher Umgang mit knappen Ressourcen wie Zeit und Arbeitskraft notwendig ist, um dem Patienten die bestmögliche Behandlung zu bieten. Folglich ist es von großem Interesse, überflüssige bürokratische Belastung zu minimieren und damit die Zeit, die der Arzt mit dem Patienten verbringen kann, zu maximieren. Der Gesundheitswissenschaftler Avedis Donabedian differenziert dabei drei Aspekte: Struktur-, Prozess- und Ergebnisqualität (Birkner et al 2003).

Die Strukturqualität beschreibt Rahmenbedingungen. Im Hinblick auf die Attestausstellung lassen sich hier die Einarbeitung des Arztes und der Praxisassistenz als personelle Ressourcen und Vorlagen sowie eine geeignete Praxissoftware als materielle Ressourcen anführen.

Die Prozessqualität umfasst alle Aktivitäten, die zum Endprodukt beitragen, beispielsweise die Untersuchungen, die zur Ausstellung des Attests notwendig sind, sowie die Formulierung des Attests.

Die Ergebnisqualität bewertet den Gesundheitszustand und die Zufriedenheit des Patienten. Ein wichtiger Einflussfaktor dafür sind die Zeit und die Aufmerksamkeit, die der Arzt für den Patienten aufbringen kann. 


\section{Einfluss auf Arzt und Patienten}

Obwohl Befragungen zeigen, dass die Patienten insgesamt zufrieden mit ihrem Arzt sind und seine Leistungen hoch anerkennen, bemängeln sie, dass der Arzt zu wenig Zeit für sie hat (Statistisches Bundesamt 2015).

Die Dokumentation im Gesundheitssystem macht Behandlungsabläufe transparent und nachvollziehbar und ist für die Qualitätssicherung unerlässlich. Dabei muss stets überprüft werden, in welchem Ausmaß der Patient davon profitiert (Statistisches Bundesamt 2015). Pro Praxis ist eine Person durchschnittlich 96 Tage mit der Bewältigung bürokratischer Vorgaben beschäftigt. „Das ist wertvolle Zeit, die den Ärzten und Zahnärzten für die Behandlung fehlt“ (Kammer 2015, S.2). Der bürokratische Aufwand sollte in einem angemessen Rahmen gehalten werden, sodass er die Zeit, die für die eigentliche Behandlung des Patienten aufgewendet wird, nicht einschränkt.

Ein geringer Verwaltungsaufwand verbessert die Arbeitsbedingungen in der Praxis. Davon profitiert auf lange Sicht nicht nur der Arzt. Das Befinden des Arztes rückt als Einflussfaktor auf die Qualität seiner Leistung zunehmend in den Fokus der Wissenschaft. Dem wird so viel Bedeutung beigemessen, dass das Wohlergehen als Qualitätsindikator der Zukunft des Gesundheitssystems diskutiert wird (Wallace et al 2009).

Verbesserungsmaßnahmen für die Zufriedenheit und Befindlichkeit der Ärzte, könnten nicht nur ihnen selbst, sondern auch dem Patienten zu Gute kommen (Williams et al 2003, Shanafelt et al 2005). Voraussetzung für das Wohlergehen ist unter anderem ein stimulierendes Arbeitsumfeld, das Entwicklung und Erfolg ermöglicht (Shanafelt et al 2005). Durch die weltweite Tendenz zum Ärztemangel kommt einem solchen Arbeitsumfeld besondere Bedeutung zu. Schlechte Arbeitsbedingungen führen dazu, dass mehr qualifizierte Mediziner in andere Bereiche abwandern und wirken sich außerdem negativ auf Produktivität, Effizienz und Versorgungsqualität aus (Cooper et al 2002, Virtanen et al 2008).

Auch aus wirtschaftlicher Sicht ist der Erhalt der ärztlichen Arbeitskraft von dringendem Interesse: Ärzte mit Burnout Symptomen zeigen erwartungsgemäß eine höhere Tendenz zu krankheitsbedingten Fehltagen, Arbeitsplatzwechsel und frühe Berentung. Außerdem verzeichnen sie einen geringeren Umsatz, verbringen weniger Zeit mit Patienten und ordnen mehr unnötige Untersuchungen an (Linzer et al 2001). 
Selbstverständlich liegen verbesserte Arbeitsbedingungen im persönlichen und professionellen Interesse des Arztes. Dass die Überlastung mit einem höheren Risiko für Burnout einhergeht (Shanafelt et al 2002) und sich negativ auf die Aufmerksamkeit auswirkt, zeigen Studien, die die Fehlerraten in langen Arbeitsschichten betrachten. Ärzte machten dabei signifikant mehr Flüchtigkeitsfehler (Lockley et al 2004), wenn sie Schichten über 16 Stunden arbeiten und schwerwiegendere medizinische Fehler in 24Stunden-Schichten (Landrigan et al 2004).

Nicht nur die Dauer der Arbeit, sondern auch übermäßige Anforderungen, eine Flut von Informationen in kürzester Zeit zu verarbeiten, haben negativen Einfluss auf die Arbeitsqualität (Levin et al 2007). Das Reglement von Verwaltung und Kosten durch die Regierung setzt der Autonomie des Arztes immer engere Grenzen und beeinflusst so die Befindlichkeit des Arztes (Dunstone et al 2001, Gross et al 2007, Williams et al 2007).

Es ist also von größtem Interesse, die Verwaltung, welche die knapp begrenzte Arbeitskraft hoch qualifizierter Mediziner in Anspruch nimmt, auf das notwendige Minimum zu reduzieren, um Patienten die bestmögliche Versorgung mit der dafür notwendigen Zeit und Aufmerksamkeit zu ermöglichen. 


\section{Ziele der Untersuchung}

Für die Ausstellung von Attesten gibt es keine verbindlichen formalen oder inhaltlichen Standards und auch für die notwendige Qualifikation der Ärzte gibt es keine Vorgaben. Ziel der Untersuchung ist es, verschiedene Aspekte der Attestausstellung wie Vorgehensweisen, Zeitaufwand und Abrechnungspraxis zu beleuchten und Schwierigkeiten und Verbesserungswünsche zu identifizieren (Ertl L 2016).

In Experteninterviews wurde eruiert, welches Potential hausärztlich tätige Ärzte in einer Standardisierung sehen, welche Aspekte es bei der Attestausstellung zu beachten gilt, welche Vorgehensweisen aktuell angewendet werden und welche Verbesserungen möglich wären (Ertl L 2016).

Argumente für eine Vereinheitlichung wurden auch im Fragebogen überprüft. Außerdem wurden konkrete Zahlen zu Zeitaufwand, Vergütung und Attestarten ermittelt, um das Potential und den Bedarf einer Vereinheitlichung zu quantifizieren (Ertl L 2016).

Mit der Untersuchung soll ein Überblick über die aktuelle Situation gegeben werden und eruiert werden, inwieweit eine Standardisierung von Attesten die alltägliche Aufgabe erleichtern könnte. Unter Einbeziehung einschlägiger Erkenntnisse aus Fragebogen und Gesprächen bezüglich Defiziten sollen mit Hilfe von Beispielen bestehender Attestvorlagen konkrete, konstruktive Verbesserungsvorschläge erarbeitet werden. 


\section{Methoden}

Ziel der Untersuchung war es, eine erste Übersicht zu Indikationen, Relevanz und Hindernissen von Attesten zu bieten. Um die aktuelle Situation zu erfassen und gleichzeitig Verbesserungsansätze zu generieren eignete sich die Vorgehensweise einer Mixed Method Research (Leech, Onuwuegbuzie 2010). Mit dem Fragebogen konnten konkrete Zahlen zu Aufwand und Vergütung ermittelt werden, während die Gespräche ermöglichten, Defizite aufzudecken und Lösungsstrategien zu identifizieren (Ertl L 2016). Die Erhebungsmethoden des starren Schemas im Fragebogen und der flexiblen Form des Gesprächs haben sich so optimal ergänzt (Onuwuegbuzie, Leech 2004).

\subsection{Ethikvotum \& Datenschutz}

Fragebogen und Gesprächsleitfaden wurden der Ethikkommission vorgelegt und nach Erteilung eines positiven Votums freigegeben. Dem Fragebogen waren eine Einwilligungserklärung und eine Probandeninformation mit Informationen zu Teilnahme, Thema der Umfrage und Verwendung der Daten beigefügt (siehe Anhang).

Zur Gesprächsteilnahme und -aufzeichnung wurde eine zusätzliche Einwilligungserklärung eingeholt.

Der Online Fragebogen enthielt einen zusätzlichen Datenverwendungshinweis.

Die ausgefültten Fragebögen und Expertengespräche wurden anonymisiert und streng vertraulich behandelt. Die Erhebung, Verarbeitung und Speicherung aller Daten wurde ausschließlich für die Dissertation verwendet (Ertl L 2016). 


\subsection{Ablauf der Umfrage}

Die folgende Abbildung gibt einen Überblick zum zeitlichen Ablauf der Studie.

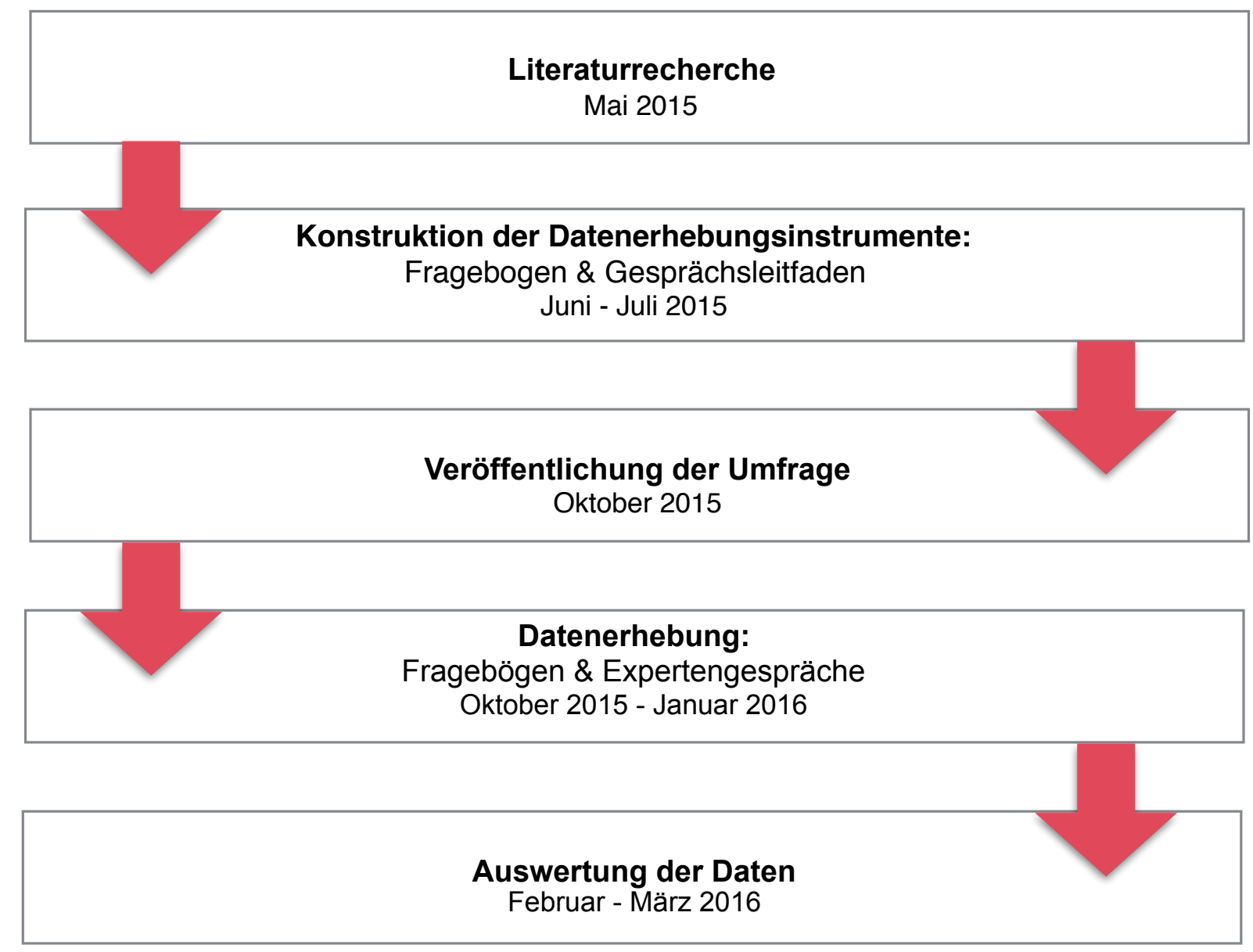

Abbildung 1: Zeitlicher Ablauf der Studie

\section{Literaturrecherche}

Zum Thema Atteste und Untersuchungen konnten im Dissertationsverzeichnis der Deutschen Nationalbibliothek, Ludwig-Maximilians-Universität, und der Technischen Universität München keine anderen Arbeiten gefunden werden. Literatur zum Thema wurde über die wissenschaftlichen Datenbanken PubMed, Medline und Web Of Science und das Onlinearchive medizinischer Fachzeitschriften recherchiert. Während die 
Konsequenzen des steigenden Verwaltungsaufwands zunehmend diskutiert werden, konnten keine Untersuchungen, die sich explizit mit Attesten beschäftigen, gefunden werden.

Der Deutsche Hausärzteverband und die KVB wurden kontaktiert, um festzustellen, ob bereits Zahlen zu Zeitaufwand, Art und Verteilung von Attesten in der allgemeinärztlichen Praxis vorliegen. Dies wurde von beiden Stellen verneint. Die KVB wies auf zwei Projekte zum Thema Bürokratieabbau hin: In „Bessere Regulierung in der ambulanten Versorgung“ ermittelte die Kassenärztliche Vereinigung Westfalen-Lippe eine erste Messung der bürokratischen Belastungen von Vertragsärzten und Psychotherapeuten in WestfalenLippe. In „Mehr Zeit für Behandlung - Vereinfachung von Verfahren und Prozessen in Arztund Zahnarztpraxen“ hat der Normenkontrollrat (NKR) im Auftrag der Bundesregierung erstmals die bürokratische Belastung der niedergelassener Ärzte erhoben. Das Projekt erfolgte in drei Phasen (Normenkontrollrat 2015), die dem Aufbau der Umfrage zum Thema Atteste ähneln:

- Datenerhebung zum bürokratischen Aufwand

- Identifizierung besonders zeitintensiver Informationspflichten

- Erarbeiten von Verbesserungsansätzen

Das Projekt war zur Zeit der anfänglichen Recherche in der letzten Phase und wurde im August 2015 abgeschlossen. Aus der Literaturrecherche ging hervor, dass das Thema Atteste im Hinblick auf Bürokratieabbau als gesundheitsökonomisches Ziel ein hochaktuelles Thema ist, zu dem kürzlich erstmals in zwei Projekten Informationen gesammelt wurden. Atteste wurden als Teil der Informationspflichten in der Praxis mituntersucht, waren jedoch nicht der Fokus der Erhebungen.

Zusätzlich wurden Beiträge zum Thema im Diskussionsforum Listserver Allgemeinmedizin gesucht. Die große Zahl an Beiträgen zu Attestanfragen spricht für die aktuelle Bedeutung des Themas in der Praxis.

Der Stand der Literatur muss bei der Formulierung der Fragestellung und der Auswahl der Erhebungsmethoden berücksichtigt werden (Leech, Onuwuegbuzie 2010). Auf Basis der Recherche wurden Fragebogen und Gesprächsleitfaden mit dem erstmaligen Fokus auf Atteste erstellt. 


\section{Rekrutierung und Rücklaufquote}

Um eine möglichst große Teilnehmerzahl zu erreichen, wurde der Fragebogen über verschiedene Kanäle verteilt (Ertl L 2016). Durch die Vielzahl der Verteiler lässt sich die Zahl der hausärztlich tätigen Ärzte, welche die Bitte um Teilnahme an der Umfrage gelesen haben, nicht abschätzen. Deshalb ist keine Rücklaufquote zu ermitteln. Als Verteiler wurden E-Mail, Fachzeitschriften, Online Portale und Newsletter genutzt. Die folgende Tabelle zeigt die Veröffentlichungen im Überblick.

Abbildung 2: Veröffentlichung über verschiedene Medien im zeitlichen Verlauf

\begin{tabular}{|l|l|l|l|l|}
\hline & E-Mail & Newsletter & Online Portal & Fachzeitschriften \\
\hline $\begin{array}{l}\text { Oktober } \\
2015\end{array}$ & $\begin{array}{l}\text { Lehrärzte der } \\
\text { Ludwigs-Maximilians- } \\
\text { Universität } \\
\text { Lehrärzte der Technischen } \\
\text { Universität München }\end{array}$ & & & \\
\hline $\begin{array}{l}\text { November } \\
2015\end{array}$ & & BHAEV 36/15 & Der Allgemeinarzt & \\
\hline $\begin{array}{l}\text { Dezember } \\
2015\end{array}$ & & $\begin{array}{l}\text { Allgemeinarzt } \\
\text { Online }\end{array}$ & $\begin{array}{l}\text { Der } \\
\text { niedergelassene } \\
\text { Arzt }\end{array}$ & $\begin{array}{l}\text { Der } \\
\text { niedergelassene } \\
\text { Arzt (12/15) }\end{array}$ \\
\hline $\begin{array}{l}\text { Januar } \\
2016\end{array}$ & & $\begin{array}{l}\text { Springer Update } \\
\text { Allgemeinmedizin }\end{array}$ & Springer Medizin & $\begin{array}{l}\text { Zeitschrift für } \\
\text { Allgemeinmedizin } \\
(12 / 15)\end{array}$ \\
\hline
\end{tabular}




\subsection{Datenerhebung}

\section{Fragebogen}

Der Fragebogen (s. Anhang) wurde für eine quantitative Auswertung angelegt. Dabei gab es drei Fragetypen für geschlossene Fragen:

- Fragen nach Schätzwerten: Die Werte wurden in Felder mit vorgegebener Einheit (Minuten, Stück, Prozent) eingetragen.

- Multiple Choice Fragen

- Skalierte Fragen: Um Bewertungen (Empfinden, Bedeutung) greifbar zu machen, wurden die Antwortmöglichkeiten skaliert. Dabei wurde ein 4-stufiges Schema gewählt, um Neutralmeinungen auszuschließen.

Bei einigen Fragen war zusätzlich die Möglichkeit eines freien Kommentars gegeben.

Der Fragebogen umfasst die Themenkomplexe Bedeutung der Attestausstellung im Praxisalltag, Argumente für die Standardisierung, Zusammensetzung und Zahl der Attestanfragen, Zeitaufwand, bestehende Vorgehensweisen bei Attestausstellung und Abrechnung und Vergütung.

Die geplante Fallzahl wurde nach Beratung durch einen Mitarbeiters des Instituts für medizinische Informationsverarbeitung, Biometrie und Epidemiologie auf $n=100$ festgelegt.

Der Fragebogen wurde als PDF Dokument per E-Mail versendet und konnte nach der Bearbeitung in Papierform per Email oder Fax zurückgesendet werden. Zusätzlich wurde eine Online Version des Fragebogens mit der Online-Umfrage-Applikation LimeSurvey erstellt. Die verschiedenen Bearbeitungsmöglichkeiten sollten die Teilnahme an der Umfrage erleichtern, um möglichst viele Ärzte zu erreichen. Von den 172 vollständig beantworteten Fragebögen wurden 15 in Papierform und 157 online beantwortet (Ertl L 2016). 


\section{Expertengespräche}

Die Teilnehmer wurden am Ende der Umfrage gebeten, ihre Bereitschaft für ein Gespräch durch eine Email mit Kontaktdetails zu signalisieren (um die Anonymität zu gewährleisten, wurden die Daten nicht im Fragebogen erfragt) (Ertl L 2016). Daraufhin wurde ein Gesprächstermin vereinbart.

Das Gespräch wurde nach Einwilligung der Teilnehmer aufgezeichnet. Dadurch wurde ein schnellerer Ablauf ermöglicht und sichergestellt, dass kein Wort des Gesprächs der Aufzeichnung entging.

Der Gesprächsleitfaden (s. Anhang) wurde auf eine qualitative Auswertung angelegt. Dabei sollten Themen, die im Fragebogen teilweise bereits angeschnitten worden waren, in offenen Fragen vertieft werden. Außerdem sollten Aspekte der Attestausstellung herausgearbeitet werden, die dem Gesprächspartner besonders wichtig erscheinen. Zur Erstellung des Gesprächsleitfadens beriet ein Mitarbeiter des Instituts für Didaktik und Ausbildungsforschung in der Medizin. Eine Teilnehmerzahl von 10 bis 30 war geplant.

Die Form des Gesprächs als explorative Methode wurde gewählt, um einen ersten Überblick über Relevanz und Hindernisse in der hausärztlichen Praxis zu schaffen. Diese Vorgehensweise bietet sich an, wenn ein Gegenstand bislang wenig untersucht ist (Flick et al. 2000, S.78).

Der halbstrukturierte Leitfaden sollte das Grundgerüst für die Gespräche bieten. Damit wird sichergestellt, dass die wichtigsten Themen angesprochen werden und die Gespräche untereinander vergleichbar sind. Gleichzeitig blieb genug Raum, um auf den Gesprächspartner und seine individuellen Schwerpunkte einzugehen. Der Gesprächsleitfaden ist also „eher eine Richtschnur, die die unbedingt zu stellenden Fragen enthält" (Gläser, Laudel 2009, S.42) als ein starres Programm.

Von den 31 Gesprächen wurden fünf persönlich und 26 per Telefon geführt (Ertl L 2016). Im Setting des persönlichen Gegenübers entstanden besonders ausführliche Gespräche. Das ist vermutlich darauf zurückzuführen, dass durch das persönliche Treffen eine ruhigere Atmosphäre gegeben war als bei den meisten Telefonaten. Aus organisatorischen Gründen konnten nicht alle Gespräche persönlich geführt werden. 
Die Gesprächsteilnehmer äußerten reges Interesse an den Ergebnissen der Umfrage.

Dafür wurden die wichtigsten Ergebnisse und konkreten Handlungsempfehlungen in Kurzform zusammengefasst. Die Übersicht findet sich im Anhang.

\section{Attestbeispiele}

In Fragebogen und Gespräch wurden die Teilnehmer gebeten, anonymisierte

Beispielatteste aus der eigenen Praxis zur Verfügung zu stellen. Diese konnten per E-Mail, Fax oder Post gesendet werden. Insgesamt wurden 56 verschiedene Atteste von 10 Ärzten zugesandt. Eine tabellarische Übersicht der Beispielatteste findet sich im Anhang.

\subsection{Auswertung \& Statistik}

\section{Fragebogen}

Die Daten des Fragebogens wurden mit Hilfe der Statistik-Software Statistical Package for the Social Sciences (SPSS, Version 23) analysiert (Ertl L 2016). Dabei wurden Methoden der deskriptiven Statistik angewandt (Häufigkeitsverteilung, Mittelwert und Standardabweichung bzw. Konfidenzintervall, Minima und Maxima). Für den statistischen Vergleich wurde der T-Test für verbundene Stichproben verwendet. Ein P-Wert $<0.05$ wurde als signifikant bewertet. Als Ausgabeformate wurden Tabellen und Balkendiagramme gewählt, die mit dem Programm Pages (Version 5) erstellt wurden. Die Kommentare wurden in Textform zusammengefasst.

Zur Auswertung der Ergebnisse wurden Berater des Instituts für medizinische Informationsverarbeitung, Biometrie und Epidemiologie und vom Institut für Medizinische Statistik und Epidemiologie in München konsultiert.

Insgesamt wurden 172 Fragebögen vollständig ausgefüllt. Die 6 unvollständigen OnlineFragebögen, bei denen jeweils höchstens die ersten vier Fragen beantwortet wurden, wurden nicht in die Auswertung aufgenommen. 


\section{Expertengespräche}

Die Interviews sollten primär als exploratives Erhebungsinstrument zur Darstellung der aktuellen Situation dienen. Zur Auswertung der Ergebnisse wurden Berater am Institut für Didaktik und Ausbildungsforschung in der Medizin und am Institut für Ethik, Geschichte und Theorie der Medizin zu Rate gezogen.

Der erste Teil der Auswertung fand direkt im Anschluss an das jeweilige Gespräch statt (Mayring 2002). Dabei wurden die Interviews zusammengefasst, neue Erkenntnisse hervorgehoben und weiterführende Fragen notiert. Das Verfassen von Memos ist ein grundlegendes Element der Grounded-Theory-Methodologie (Glaser, Strauss 2012, S.45), durch das die Forschungsfragen zunehmend konkretisiert werden sollen. Zur abschließenden Auswertung mit Methoden der qualitativen Inhaltsanalyse wurden die Interviews mithilfe der Tonaufnahmen verschriftlicht.

Ziel der Auswertung des Textmaterials ist eine Ordnung nach empirisch und theoretisch sinnvollen Gesichtspunkten, um eine strukturierte Wiedergabe zu ermöglichen (Barton, Lazarsfeld 1979).

Dabei wurden Methoden der qualitativen Inhaltsanalyse angewendet, um eine möglichst genaue Abbildung des Materials ohne Verzerrung durch den Untersucher zu ermöglichen (Mayring 2010, S.84). In der Inhaltsanalysetechnik der „Zusammenfassung und Kategoriebildung" (Mayring 2010, S.67) werden alle inhaltstragenden Textstellen in Kurzform paraphrasiert und auf die gleiche Abstraktionsebene gebracht. In einer ersten Reduktion werden bedeutungsgleiche Paraphrasen zusammengefasst. Im zweiten Schritt werden Paraphrasen mit gleichem Gegenstand und gleichen Aussagen (Bündelung) und Paraphrasen mit gleichem Gegenstand und verschiedenen Aussagen (Konstruktion/ Integration) zusammengefasst.

Auf Basis dieser Zusammenfassung werden Kategorien im Sinne einer „offenen Codierung" aus dem Material abgeleitet (Strauss 1987, Strauss, Corbin 1990). Durch die induktive Kategoriebildung konnten die Ergebnisse der Expertengespräche strukturiert und übersichtlich aufbereitet werden. 


\section{Problem - \& Verbesserungspotential}

Um Erläuterungen der Problematik, konstruktive Kritik, bestehende Vorgehensweisen und Verbesserungsvorschläge strukturiert wiederzugeben, wurden die Kategorien Definition, Vollständigkeit, Verantwortung, Medizinische Relevanz, Datenschutz und Schweigepflicht, Vorlagen und Abrechnung aus dem gesamten Material abgeleitet. Eine tabellarische Übersicht über die Kategorien mit Beispielen aus den Gesprächen findet sich im Ergebnisteil.

\section{Einstellung und Umgang}

Experteninterviews eignen sich dazu, Ansichten und Einstellung der Befragten zu erfassen (Mayring 2010, S.33). Um die Einstellung der Gesprächspartner gegenüber der Standardisierung von Attesten herauszuarbeiten, wurden zusätzlich die Kategorien Unzufriedenheit mit der aktuellen Situation, Wunsch nach Veränderung, Offenheit für Verbesserung und eigene Lösungsansätze abgeleitet. Um möglichst ausführliches Material für diese vertiefende Analyse zu verwenden, wurden die 5 persönlichen Interviews und die 5 längsten Telefoninterviews ausgewählt. Eine tabellarische Übersicht über die Kategorien mit Beispielen aus den Gesprächen findet sich im Ergebnisteil.

\section{Aktuelle Situation}

Um die aktuelle Situation zu erfassen, wurden die Punkte Vorlagen, Abrechnung, Untersuchungen und Zuständigkeit standardmäßig abgefragt. Das Kategoriesystem für die Auswertung wurde in diesem Fall deduktiv aus dem Gesprächsleitfaden abgeleitet. Anschließend wurden Textstellen im Sinne einer Kodierung eingeordnet. Die Frequenzanalyse ermöglicht eine objektive Darstellung und Interpretation der Ergebnisse (Mayring 2010, S.15).

Da die Fallzahl von 31 für eine statistische Auswertung nicht ausreicht, wurden absolute Häufigkeiten angegeben, um den Trend der Antworten in Zahlen zu fassen. Eine 
tabellarische Übersicht über die Kategorien mit absoluten Häufigkeiten findet sich im Ergebnisteil.

\section{Attestbeispiele}

Auf Grundlage der Beispiele konnte ein Überblick über den Aufbau bestehender Attestvorlagen gewonnen werden. Atteste, die auf eine Grundproblematik der Attestausstellung (z.B. unklare Definition in der Anfrage) eingehen, wurden identifiziert und in die Verbesserungsvorschläge integriert. Eine tabellarische Übersicht der eingereichten Atteste findet sich im Anhang. 


\section{Ergebnisse}

Im Folgenden werden die Ergebnisse des Fragebogens zusammengefasst und mit Hilfe von Diagrammen visualisiert. Die Ergebnisse der Expertengespräche und Attestbeispiele werden in tabellarischer Form dargestellt.

\subsection{Fragebogen}

Die maximale Teilnehmerzahl ist $n=172$, bei fehlenden Angaben kann diese abweichen. Der vollständige Fragebogen und die tabellarische Auflistung der Ergebnisse mit Lage und Streuungsparametern findet sich im Anhang.

\section{Bedeutung der Attestausstellung im Praxisalltag}

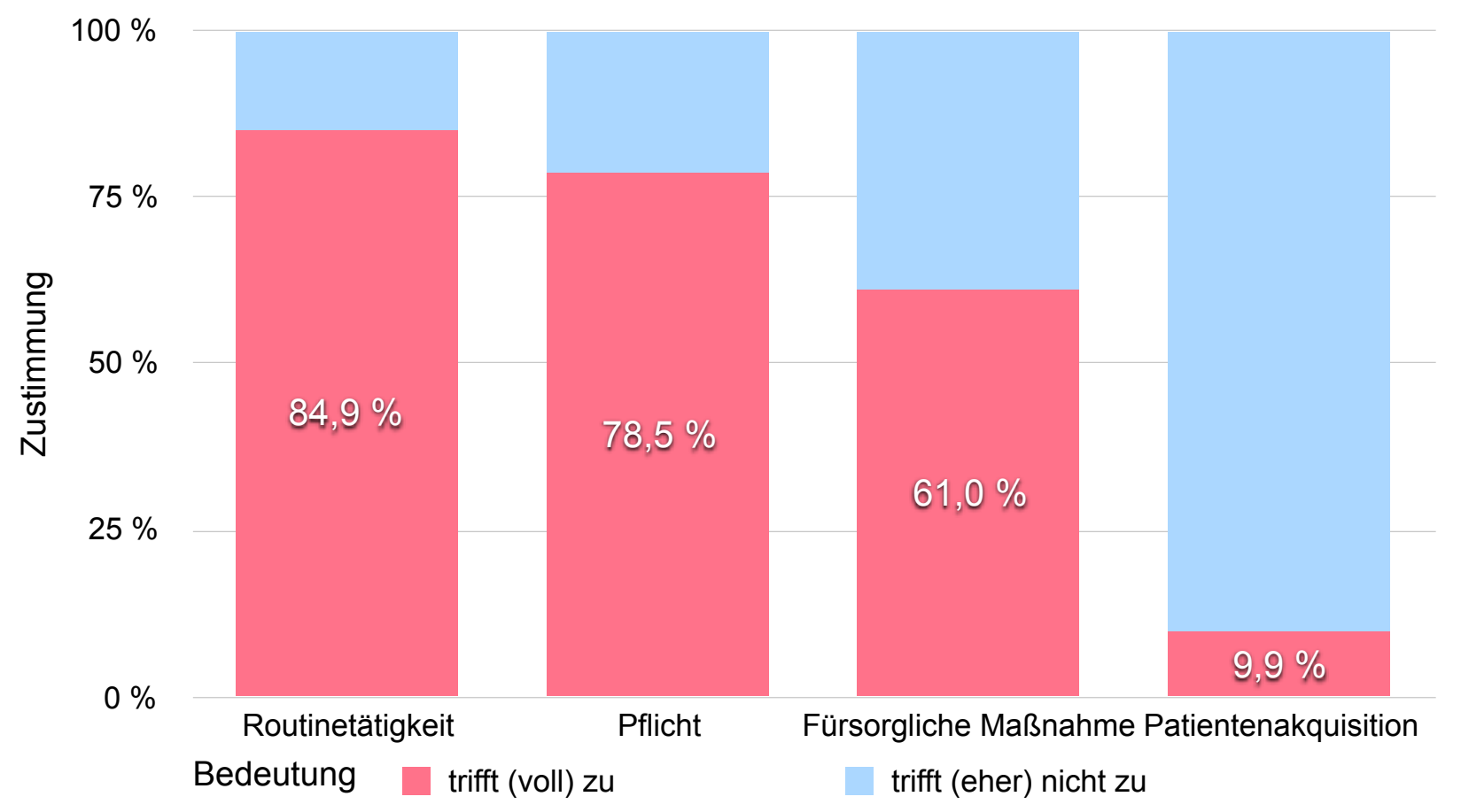

Abbildung 3: Unter welche Kategorie fällt die Ausstellung von Attesten nach Ihrem persönlichen Empfinden?

Die meisten Ärzte nehmen die Ausstellung von Attesten vorwiegend als Routinetätigkeit und Pflicht wahr. 


\section{Argumente für die Standardisierung}

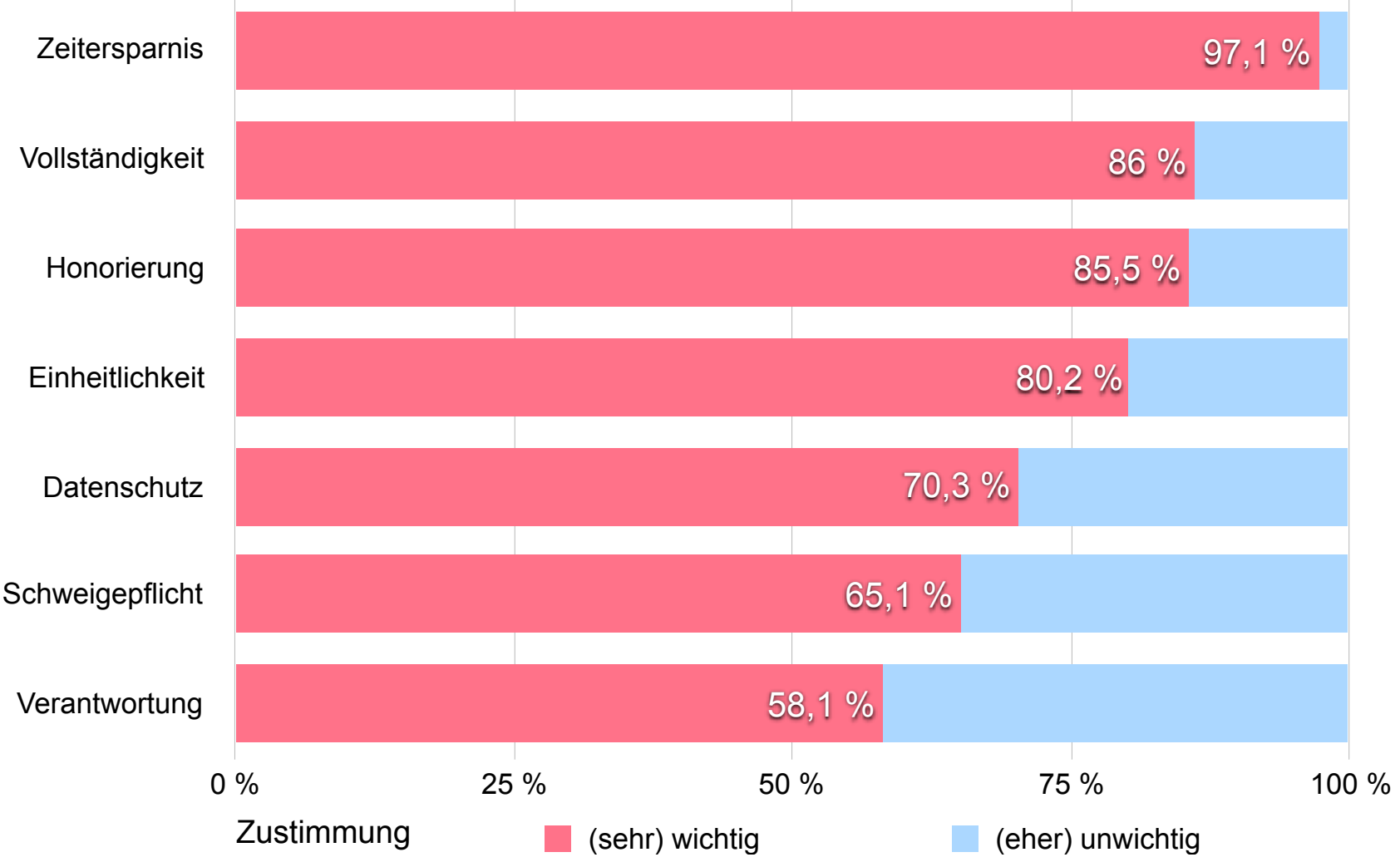

Abbildung 4: Wie wichtig sind die folgenden Argumente für sie und sprechen damit für eine Standardisierung?

Das mit Abstand wichtigste Argument ist die Zeitersparnis (Ertl L 2016). Zusätzlich könnten Vorlagen dazu beitragen, die Vollständigkeit sicherzustellen, eine angemessene Honorierung zu legitimieren und eine einheitliche Form innerhalb der Praxis zu wahren. 


\section{Bestehende Vorgehensweisen}

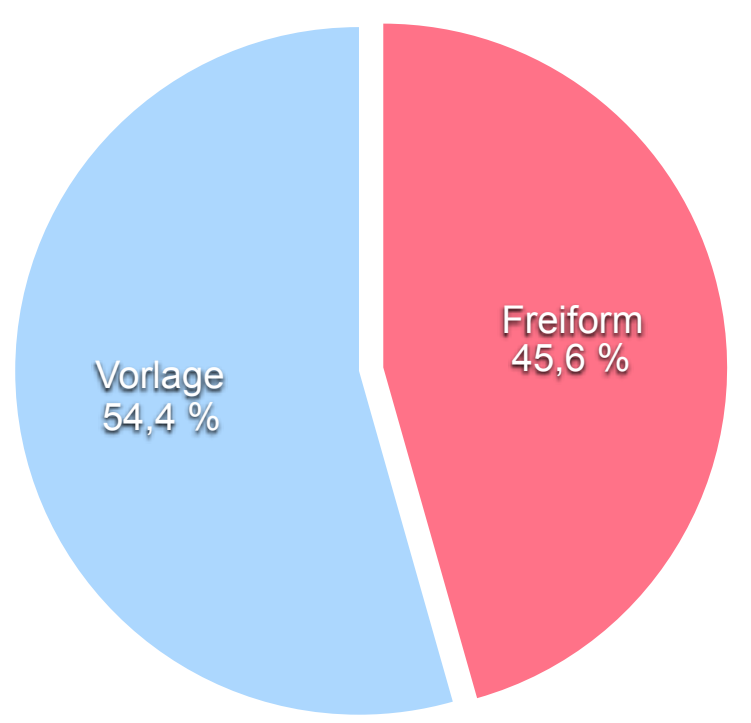

Abbildung 5: Wie werden Atteste erstellt?

Fast die Hälfte der Atteste wird ohne Vordruck angefragt und muss eigens erstellt werden (Ertl L 2016).

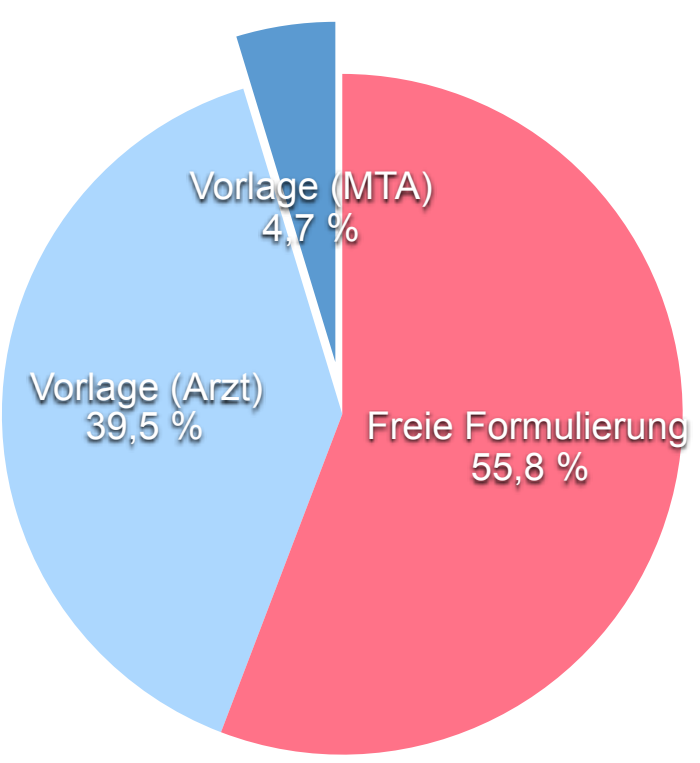

Abbildung 6: Wie werden frei formulierte Atteste erstellt?

Bei Anfragen ohne vorgegebene Vorlage formuliert mehr als die Hälfte der Ärzte jedes Mal frei. Etwa 40\% der Ärzte füllen eine eigene Vorlage aus, knapp 5\% überlassen dies ihrer Medizinisch-technischen Assistenz (MTA) (Ertl L 2016). 


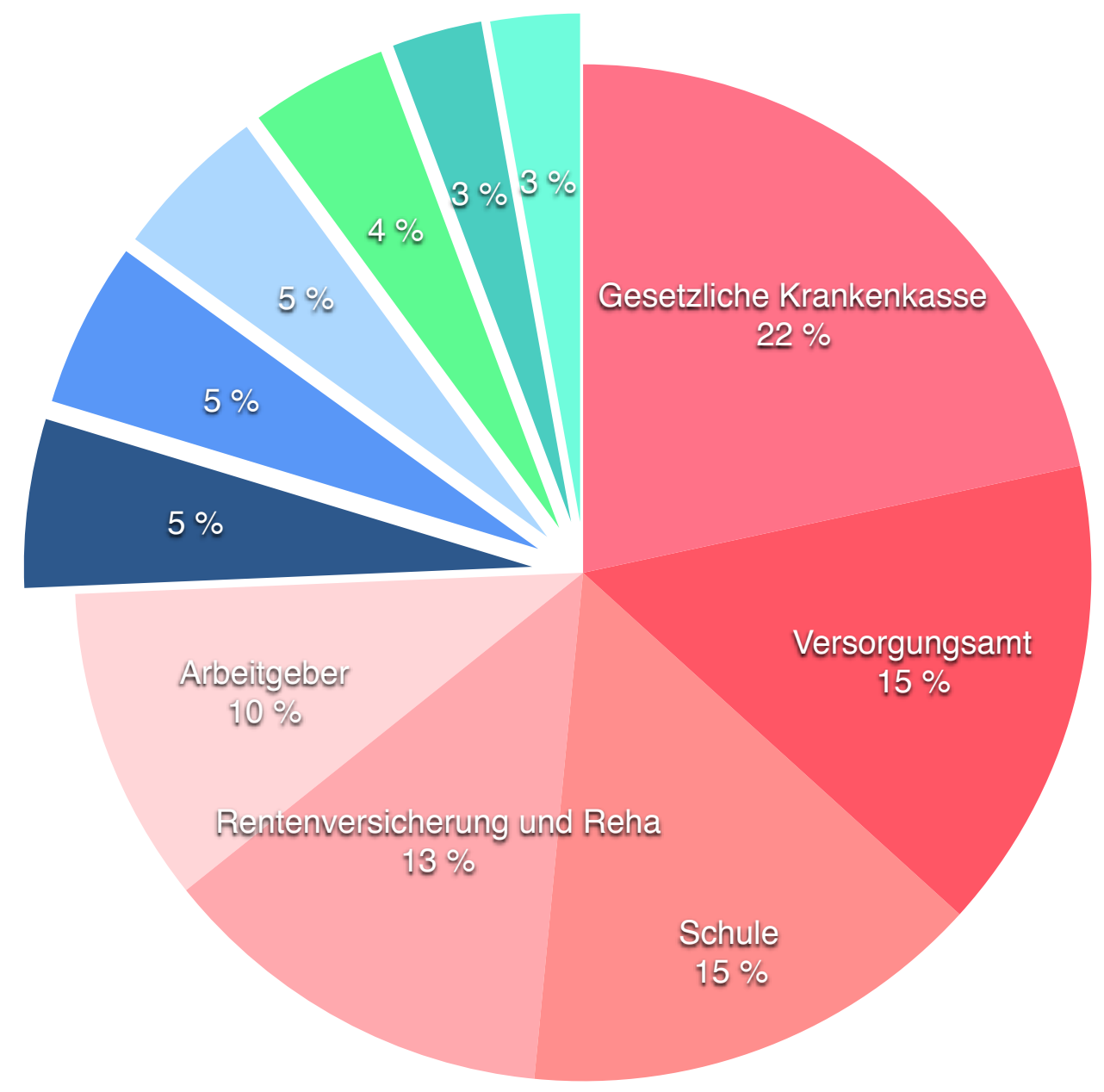

Gesetzliche Krankenkasse
Schule
Arbeitgeber
Andere Versicherungen
Sonstige
Reiserücktrittsversicherung

Versorgungsamt

Rentenversicherung und Reha

- Private Krankenkasse

Behörden

Sport

Abbildung 7: Aus welchen Anteilen setzen sich die Anfragen zusammen?

Am häufigsten werden Atteste für Gesetzliche Krankenkassen, Versorgungsamt, Schule, Rentenversicherungen und Reha-Anträge und Arbeitgeber angefordert. 


\section{Zeitaufwand pro Attest}

40

30

\section{1}

$\stackrel{5}{. \subseteq} 20$

10
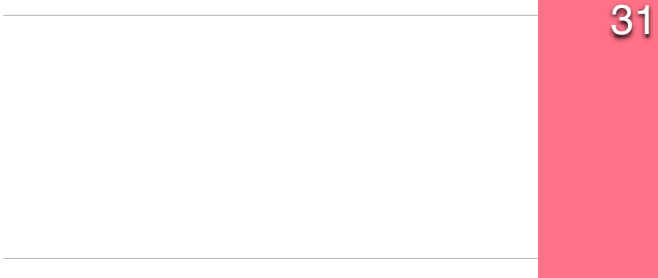

10

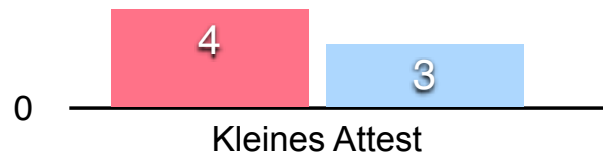

Art des Attests

Freie Formulierung

Attest mit Untersuchungen

Abbildung 8: Wie viel Zeit nimmt ein Attest durchschnittlich in Anspruch?

Das Diagramm verdeutlicht die durchschnittliche Zeit, die für die Ausstellung eines Attests notwendig ist, bei freier Formulierung und bei Verwendung von Vorlagen im Vergleich. Bei allen Attestgruppen zeigt sich eine signifikante Zeitersparnis bei der Verwendung von Vorlagen (T-Test für verbundene Stichproben, $p<0.0001)$. Dabei besteht im Mittel eine Differenz von einer Minute für kleine Atteste und 8 Minuten für Atteste mit gutachterlichem Charakter und Atteste mit Untersuchungen (Ertl L 2016). 
140

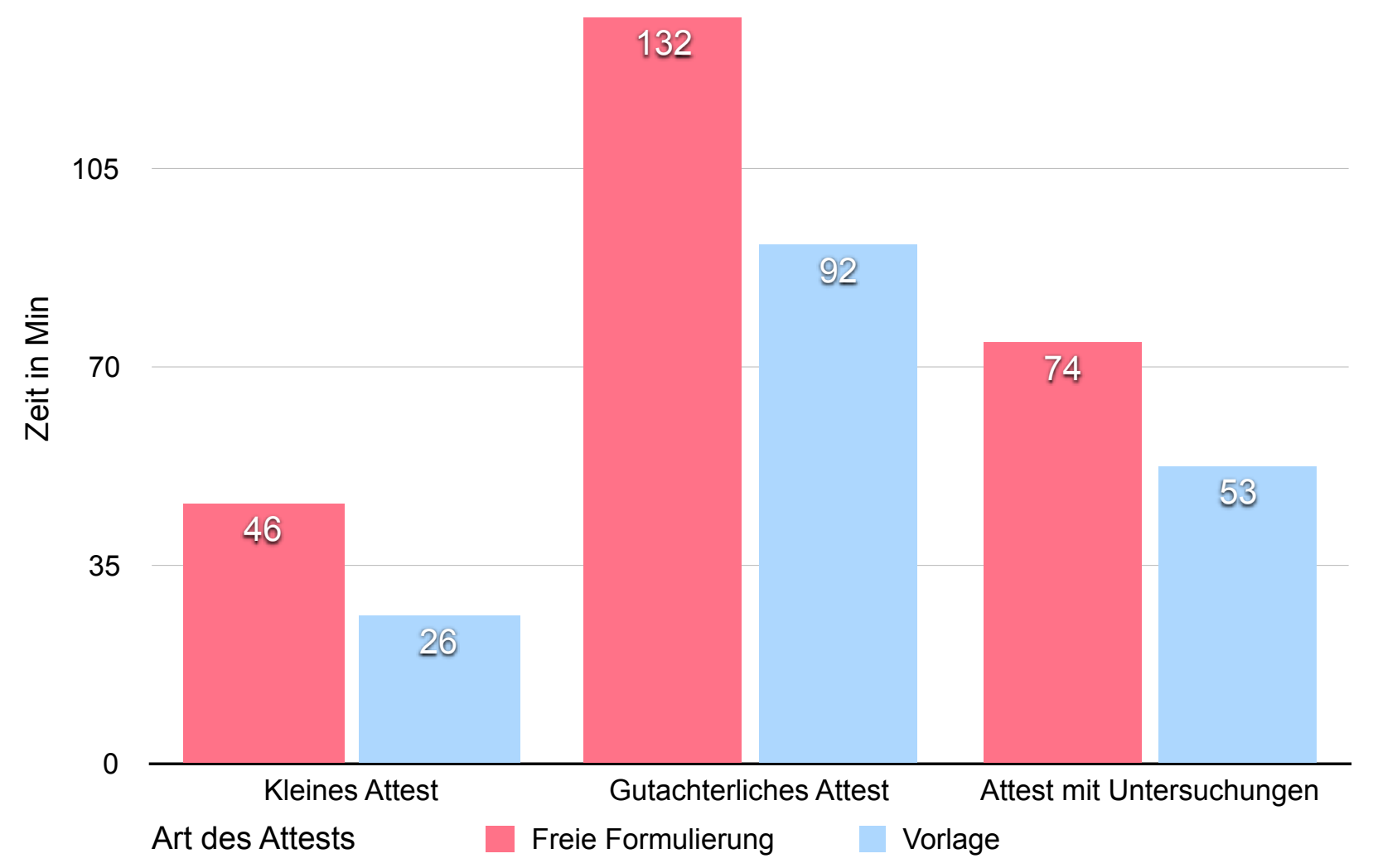

Abbildung 9: Durchschnittlicher Zeitaufwand pro Woche (Ertl L 2016)

Der durchschnittliche Zeitaufwand pro Woche ergibt sich aus dem Produkt von Zeitaufwand pro Attest und Zahl der nachgefragten Atteste. Pro Woche werden im Mittel 11 Atteste mit geringem Aufwand, 4 Atteste mit gutachterlichem Charakter und 3 Atteste mit Untersuchungen angefordert.

Das Diagramm zeigt den wöchentlichen Zeitaufwand bei freier Formulierung und bei der Verwendung von Vorlagen im Vergleich. Bei allen Attestgruppen zeigt sich eine signifikante Zeitersparnis bei der Verwendung von Vorlagen (T-Test für verbundene Stichproben, $p<0.001)$.

Für kleine Atteste ergibt sich eine Differenz von 20 Minuten, für Atteste mit gutachterlichem Charakter 40 Minuten und für Atteste mit Untersuchungen 21 Minuten (Ertl L 2016). 


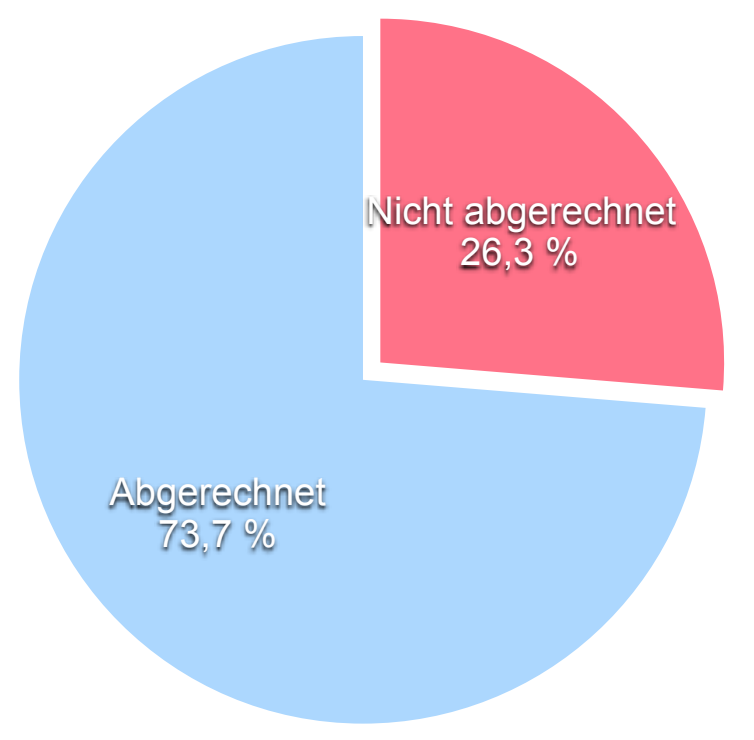

Abbildung 10: Welcher Anteil der Atteste wird allgemein abgerechnet?

Durchschnittlich werden nur drei Viertel der Atteste abgerechnet (Ertl L 2016).

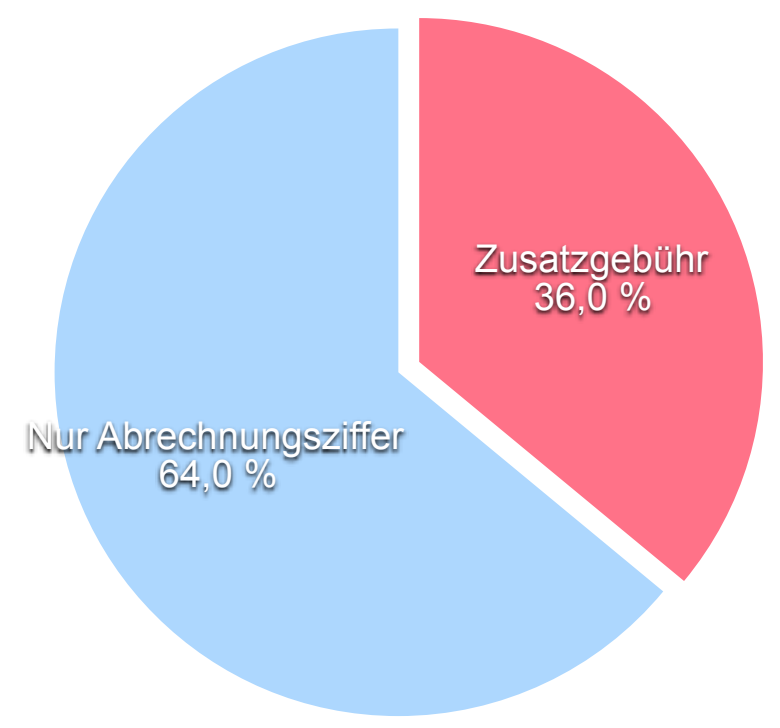

Abbildung 11: Wie rechnen Sie ab?

Etwa zwei Drittel der Ärzte rechnen dabei ausschließlich die vorgegebene Ziffer ab, die anderen erheben bei höherem Aufwand eine Zusatzgebühr.

Etwa 40\% rechnen per Barzahlung ab, weitere 40\% stellen eine Rechnung, 20\% wählen die Methode abhängig vom Einzelfall. 


\section{Vorgehen}

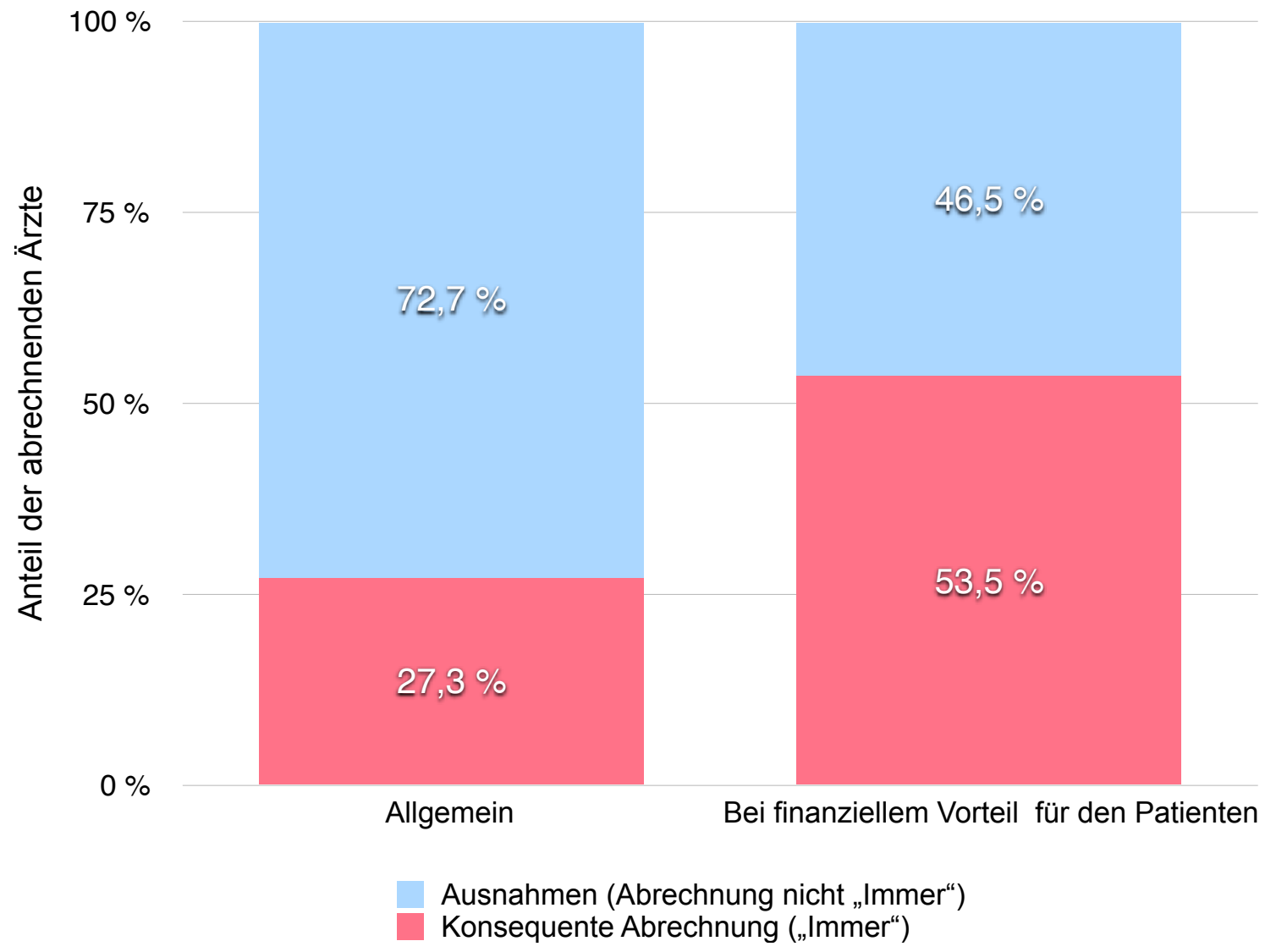

Abbildung 12: Wie oft stellen Sie Atteste allgemein in Rechnung? Wie oft stellen Sie Atteste in Rechnung, wenn der Patient primär einen finanziellen Vorteil durch das Attest erlangt?

Nur ein knappes Drittel der Ärzte macht keine Ausnahmen bei der Abrechnung. Wenn dem Patienten aus dem Attest ein finanzieller Vorteil entsteht, stellen es in etwa doppelt so viele Ärzte in Rechnung.

Als Indikationen für Ausnahmen bei der Abrechnung wurden vor allem Schulatteste und soziale Indikationen genannt. Als Anlässe für ausnahmslose Abrechnung wurden aufwendige Atteste und Atteste, aus denen ein finanzieller Vorteil für den Patienten resultiert, angeführt. Für eine statistische Auswertung wurde das Kommentarfeld nicht oft genug genutzt. 


\section{Vergütung}

\section{Betrag pro Attest}

50

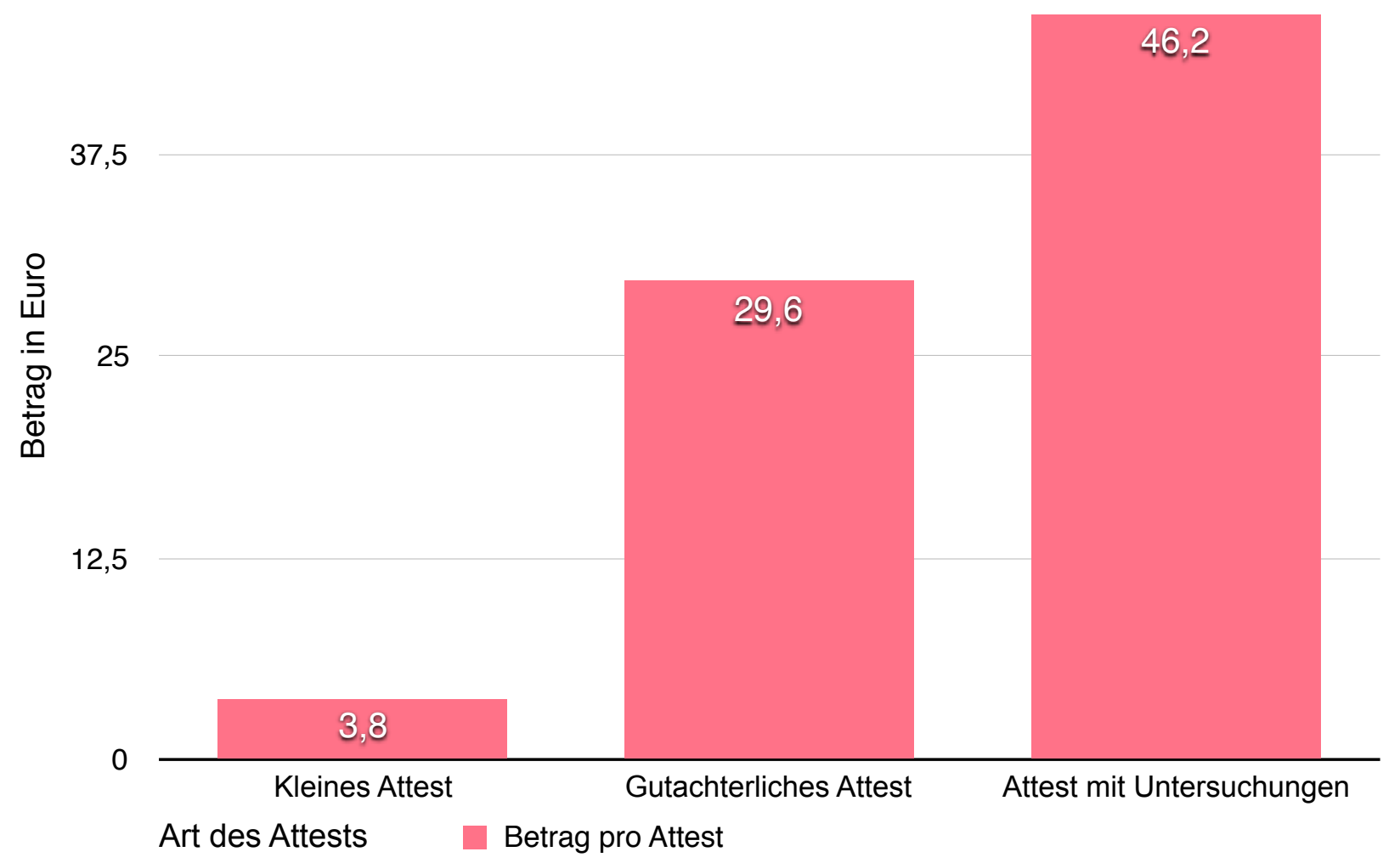

Abbildung 13: Welchen Betrag bekommen Sie durchschnittlich pro Attest erstattet?

Das Diagramm zeigt den Abrechnungsbetrag für die verschiedenen Attestgruppen (Ertl L 2016). 


\section{Stundenlohn}

200

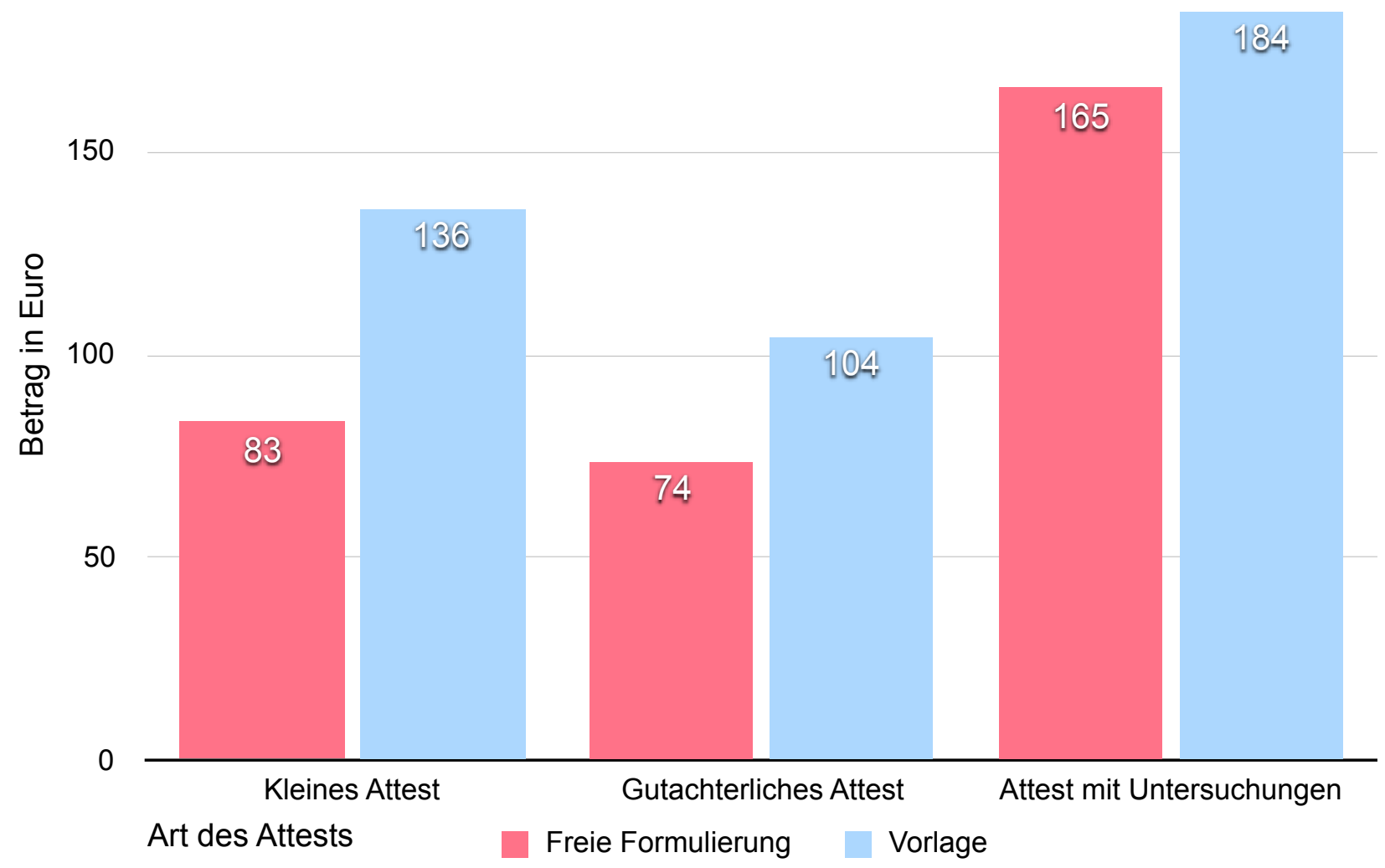

Abbildung 14: Fiktiver Stundenlohn im Vergleich (Ertl L 2016)

Zur Veranschaulichung und besseren Vergleichbarkeit lässt sich für jede Attestgruppe ein fiktiver Stundenlohn berechnen. Das Diagramm zeigt diesen Stundenlohn im Vergleich bei Verwendung von Vorlagen und bei freier Formulierung (Ertl L 2016).

Setzt man den erstatteten Betrag pro Attest ins Verhältnis mit der aufgewendeten Zeit, ist das Ergebnis bei der Verwendung von Vorlagen jeweils signifikant höher als bei freier Formulierung (T-Test für verbundene Stichproben: $p<0.001)$. 


\subsection{Gespräch}

In Form eines teilstrukturierten Interviews wurden 31 hausärztlich tätige

Allgemeinmediziner und Internisten per Telefon oder im persönlichen Gespräch befragt. Insbesondere sollten die Themen Verbesserungs- und Problempotential, Einstellung der Ärzte zur Standardisierung und aktuelle Vorgehensweisen beleuchtet werden. Die Ergebnisse werden nach Themenkomplexen strukturiert in tabellarischer Form dargestellt und anschließend im Text zusammengefasst.

\section{Problem - \& Verbesserungspotential}

Die folgende Tabelle bietet eine Übersicht zu Problem- und Verbesserungspotential in Bezug auf die Punkte Definition, Vollständigkeit, Verantwortung, Medizinische Relevanz, Datenschutz und Schweigepflicht, Vorlagen und Abrechnung.

Tabelle 1: Übersicht zu Problemen und Verbesserungsvorschlägen im Gespräch

\begin{tabular}{|c|c|c|}
\hline Definition & Verbesserungsvorschlag & Beispiele / Erläuterungen \\
\hline $\begin{array}{l}\text { Unklare Definition der } \\
\text { Bestätigung in der Anfrage }\end{array}$ & $\begin{array}{l}\text { Eigene Definitionen zu Zeitraum } \\
\text { und Grundlage der Bestätigung } \\
\text { angeben }\end{array}$ & $\begin{array}{l}\text { Frei von ansteckenden } \\
\text { Krankheiten, krank, tauglich: } \\
\text { „Anamnestisch liegen zur Zeit der } \\
\text { Attestausstellung keine Hinweise } \\
\text { auf ansteckende Erkrankungen } \\
\text { vor." (Int. 21) }\end{array}$ \\
\hline \multirow[t]{2}{*}{$\begin{array}{l}\text { Unklare Definition der } \\
\text { Entscheidungsgrundlage } \\
\text { (Untersuchungen) }\end{array}$} & $\begin{array}{l}\text { Attest sollte idealerweise } \\
\text { notwendige Untersuchungen } \\
\text { vorgeben }\end{array}$ & $\begin{array}{l}\text { Frei von ansteckenden } \\
\text { Erkrankungen: Reicht Anamnese? } \\
\text { Sollen Laborwerte bestimmt } \\
\text { werden? (Int.1, } 17,27)\end{array}$ \\
\hline & $\begin{array}{l}\text { - Erfolgte Untersuchungen genau } \\
\text { im Attest angeben }\end{array}$ & $\begin{array}{l}\text { Anamnestisch, auf Grundlage der } \\
\text { körperlichen Untersuchung, nach } \\
\text { Laborwerten (Int.1, } 6,11,27)\end{array}$ \\
\hline \multirow[t]{2}{*}{ Subjektivität der Angaben } & $\begin{array}{l}\text { - Auf mangelnde } \\
\text { Objektivierbarkeit hinweisen }\end{array}$ & $\begin{array}{l}\text { Prüfungsunfähigkeit: „Wie weit } \\
\text { kann sich der Patient } \\
\text { verstellen?“ (Int.16). }\end{array}$ \\
\hline & $\begin{array}{l}\text { - Bewusstsein für unrealistische } \\
\text { Anfragen fördern }\end{array}$ & $\begin{array}{l}\text { Eignungsuntersuchungen: Wie } \\
\text { lässt sich die Berufseignung } \\
\text { verifizieren? (Int. 21) }\end{array}$ \\
\hline
\end{tabular}




\begin{tabular}{|c|c|c|}
\hline Vollständigkeit & Verbesserungsvorschlag & Beispiele / Erläuterungen \\
\hline \multirow[t]{2}{*}{$\begin{array}{l}\text { Fehlende Vorgaben zu } \\
\text { notwendigen Angaben und } \\
\text { Untersuchungen }\end{array}$} & - Patienten einbinden & $\begin{array}{l}\text { Geeigneten Patienten selbst } \\
\text { formulieren lassen und Inhalt } \\
\text { überprüfen (Int. 28, 30) }\end{array}$ \\
\hline & $\begin{array}{l}\text { - Vollständigkeit im Voraus } \\
\text { sicherstellen }\end{array}$ & $\begin{array}{l}\text { Patienten bei anfordernder Stelle } \\
\text { nachfragen lassen (Int. 6) }\end{array}$ \\
\hline Verantwortung & Verbesserungsvorschlag & Beispiele / Erläuterungen \\
\hline Unrealistische Anfragen & $\begin{array}{l}\text { - Weitgefasste Anfragen, für } \\
\text { deren Bestätigung nicht die } \\
\text { Verantwortung übernommen } \\
\text { werden kann, durch } \\
\text { Einschränkungen relativieren }\end{array}$ & $\begin{array}{l}\text { „Forderungen unrealistischer } \\
\text { Bestätigungen“ relativieren (Int. } \\
\text { 16), z.B. } \\
\text { Tauglichkeitsbestätigungen: } \\
\text { präzise formulieren, was bestätigt } \\
\text { werden kann und auf Grundlage } \\
\text { welcher Untersuchungen (s. } \\
\text { Definition) }\end{array}$ \\
\hline \multirow[t]{2}{*}{ Übermäßige Verantwortung } & $\begin{array}{l}\text { - Bei Bestätigungen, bei denen } \\
\text { besonders große Verantwortung } \\
\text { übernommen wird auf } \\
\text { Vorbehalte und Risiken } \\
\text { hinweisen }\end{array}$ & \multirow{2}{*}{$\begin{array}{l}\text { Tauchtauglichkeit: eine } \\
\text { medizinische Komplikation könnte } \\
\text { bei mangelnder Sorgfalt oder } \\
\text { ungenauen Angaben eine } \\
\text { „menschliche und rechtliche } \\
\text { Katastrophe für den Arzt“ werden } \\
\text { (Int. 1), deshalb umsichtig } \\
\text { formulieren: „aus internistischer } \\
\text { Sicht vorbehaltlich des HNO- } \\
\text { ärztlichen Befundes..." (Int. 1) }\end{array}$} \\
\hline & $\begin{array}{l}\text { - Im Zweifel (für Teilbefund) an } \\
\text { anderen Facharzt oder Arzt mit } \\
\text { Zusatzqualifikation weiterleiten }\end{array}$ & \\
\hline Medizinische Relevanz & Verbesserungsvorschlag & Beispiele / Erläuterungen \\
\hline Objektivierbarkeit der Angaben & $\begin{array}{l}\text { - Auf Subjektivität und Grenzen } \\
\text { der eigenen Kompetenz } \\
\text { hinweisen: auch Arzt kann } \\
\text { Patientenangaben nicht } \\
\text { verifizieren }\end{array}$ & $\begin{array}{l}\text { Patient verstellt sich eventuell, um } \\
\text { Gesundheitszeugnis oder } \\
\text { Tauglichkeitsbescheinigung für } \\
\text { Beruf zu erhalten (Int. 21) }\end{array}$ \\
\hline Zuständigkeit & $\begin{array}{l}\text { - Anfragen, die die medizinische } \\
\text { Kompetenz des Arztes } \\
\text { übersteigen an andere Stelle } \\
\text { weiterleiten }\end{array}$ & $\begin{array}{l}\text { Krankheit kann vom Arzt ebenso } \\
\text { wenig verifiziert werden wie von } \\
\text { Eltern oder Lehrern, Tauglichkeit } \\
\text { für Beruf könnte vom Dienstarzt } \\
\text { vermutlich besser beurteilt werden } \\
\text { (Int. 16, 21, 30) }\end{array}$ \\
\hline Medizinische Konsequenz & $\begin{array}{l}\text { - Vernünftigen Umgang mit } \\
\text { knappen medizinischen } \\
\text { Ressourcen bei anfragender } \\
\text { Stelle und Krankenkassen } \\
\text { fördern }\end{array}$ & $\begin{array}{l}\text { Patient nimmt wegen } \\
\text { Krankschreibung für Arbeitgeber } \\
\text { oder Schule einen vollen } \\
\text { Arzttermin ohne medizinische } \\
\text { Konsequenz in Anspruch (Int. 21), } \\
\text { Im Praxisalltag „zählt jede Minute“ } \\
\text { (Int. 1) }\end{array}$ \\
\hline
\end{tabular}




\begin{tabular}{|c|c|c|}
\hline $\begin{array}{l}\text { Datenschutz und } \\
\text { Schweigepflicht }\end{array}$ & Verbesserungsvorschlag & Beispiele / Erläuterungen \\
\hline \multirow[t]{3}{*}{ Direkte Anfragen von Dritten } & $\begin{array}{l}\text { - Bei direkten Anfragen } \\
\text { automatisch Berechtigung in } \\
\text { Frage stellen }\end{array}$ & $\begin{array}{l}\text { Datenschutz bei Anfragen von } \\
\text { Versicherungen auf nicht } \\
\text { vereinbarten Vordrucken nicht } \\
\text { sichergestellt (Int. } 1,4,8,10,24 \text { ) }\end{array}$ \\
\hline & $\begin{array}{l}\text { - Mit Vordruck } \\
\text { Schweigepflichtsentbindung } \\
\text { anfordern }\end{array}$ & $\begin{array}{l}\text { Im Zweifelsfall „lieber einmal zu } \\
\text { viel“ anfordern (Int. 6) }\end{array}$ \\
\hline & $\begin{array}{l}\text { - Attest vom Patienten selbst } \\
\text { weiterleiten lassen }\end{array}$ & $\begin{array}{l}\text { Wenn der Patient das Attest } \\
\text { selbst weiterleitet, entbindet er } \\
\text { damit von der Schweigepflicht } \\
\text { (Int. 8) }\end{array}$ \\
\hline \multirow[t]{3}{*}{ Fragen nach Diagnosen } & - Vorsichtig formulieren & $\begin{array}{l}\text { Prüfungsunfähigkeits- } \\
\text { Bescheinigungen für } \\
\text { Universitäten (Int. 3, 4, 14) }\end{array}$ \\
\hline & $\begin{array}{l}\text { - Brandmarkende Diagnosen } \\
\text { nach Möglichkeit meiden }\end{array}$ & \multirow{2}{*}{$\begin{array}{l}\text { „Diagnose so wählen, dass sie für } \\
\text { den Patienten nicht } \\
\text { stigmatisierend ist“ (Int.14) }\end{array}$} \\
\hline & $\begin{array}{l}\text { - Konsequenzen für den } \\
\text { Patienten berücksichtigen }\end{array}$ & \\
\hline
\end{tabular}

\begin{tabular}{|c|c|c|}
\hline Vorlagen & Verbesserungsvorschlag & Beispiele / Erläuterungen \\
\hline \multirow[t]{7}{*}{ Standardisierung } & • Einheitliches Grundgerüst & $\begin{array}{l}\text { Einheitliche Basisvorlage mit } \\
\text { Praxis- und Patientendaten (Int. } \\
\text { 28) }\end{array}$ \\
\hline & - Vorlagen häufige kurze Atteste & $\begin{array}{l}\text { Krankschreibung, } \\
\text { Sporttauglichkeit, } \\
\text { Gesundheitszeugnis (Int. 16) }\end{array}$ \\
\hline & $\begin{array}{l}\text { - Textbausteine für häufige } \\
\text { komplexe Atteste }\end{array}$ & $\begin{array}{l}\text { Textbausteine: Anfragen vom } \\
\text { Sozialamt (Int. 28) }\end{array}$ \\
\hline & - Praxisangestellte einbinden & $\begin{array}{l}\text { Angestellte kann Attest erstellen } \\
\text { und überprüfen lassen (Int. 28) }\end{array}$ \\
\hline & $\begin{array}{l}\text { - Möglichst knapp und leicht } \\
\text { verständlich }\end{array}$ & $\begin{array}{l}\text { Vorsicht vor Mehraufwand (Int. 2, } \\
\text { 11) }\end{array}$ \\
\hline & - Individualisierbar & $\begin{array}{l}\text { Textbausteine, Kommentarfelder } \\
\text { (Int. } 2,8,28)\end{array}$ \\
\hline & $\begin{array}{l}\text { Vereinheitlichung von } \\
\text { vorgefertigten Anfragen von } \\
\text { Institutionen }\end{array}$ & $\begin{array}{l}\text { Muster für } \\
\text { Krankenkassenanfragen (Int. 28, } \\
31 \text { ) }\end{array}$ \\
\hline \multirow[t]{3}{*}{ Untersuchungs-Checklisten } & $\begin{array}{l}\text { - An Allgemeinzustand (Alter, } \\
\text { Erkrankungen) des Patienten } \\
\text { anpassbar }\end{array}$ & \multirow[t]{3}{*}{$\begin{array}{l}\text { Untersuchungskatalog / } \\
\text { Vorschläge mit } \\
\text { Auswahlmöglichkeiten (Int. 2, 3, } \\
4,11 \text { ) }\end{array}$} \\
\hline & $\begin{array}{l}\text { - An Bekanntheit des Patienten } \\
\text { anpassbar }\end{array}$ & \\
\hline & $\begin{array}{l}\text { - An bereits erfolgte } \\
\text { Untersuchungen anpassbar }\end{array}$ & \\
\hline
\end{tabular}




\begin{tabular}{|c|c|c|}
\hline Vorlagen & Verbesserungsvorschlag & Beispiele / Erläuterungen \\
\hline \multirow[t]{3}{*}{ Verbindlichkeit } & $\begin{array}{l}\text { - Verbindliche Vorlagen für } \\
\text { bessere Reproduzierbarkeit }\end{array}$ & $\begin{array}{l}\text { Standardisierung würde eine } \\
\text { bessere Vergleichbarkeit } \\
\text { ermöglichen (Int. 17) }\end{array}$ \\
\hline & $\begin{array}{l}\text { - Durch } \\
\text { Anpassungsmöglichkeiten } \\
\text { Mehraufwand vermeiden }\end{array}$ & $\begin{array}{l}\text { Vordrucke mit Kommentarfeld (Int. } \\
\text { 2, 8) und Auswahlmöglichkeiten } \\
\text { (Int. 6, 22) }\end{array}$ \\
\hline & $\begin{array}{l}\text { - Empfehlung statt imperativer } \\
\text { Charakter }\end{array}$ & $\begin{array}{l}\text { „Richtlinien“ (Int. 2) würden } \\
\text { Orientierung bieten ohne } \\
\text { zusätzlichen Aufwand zu } \\
\text { verursachen }\end{array}$ \\
\hline \multirow[t]{2}{*}{ Digitalisierung } & $\begin{array}{l}\text { Verwendung von digitalen } \\
\text { Vorlagen und Textbausteinen in } \\
\text { der Praxis }\end{array}$ & $\begin{array}{l}\text { Automatische Übernahme von } \\
\text { Patientendaten aus der } \\
\text { Praxissoftware (Int. 25, 28) }\end{array}$ \\
\hline & $\begin{array}{l}\text { - Digitalisierung von } \\
\text { vorgegebenen Vorlagen }\end{array}$ & $\begin{array}{l}\text { Digitale Muster für } \\
\text { Krankenkassenanfragen (Int. 28, } \\
\text { 31) }\end{array}$ \\
\hline Abrechnung & Verbesserungsvorschlag & Beispiele / Erläuterungen \\
\hline Patientenbewusstsein & $\begin{array}{l}\text { - Frühzeitige Aufklärung über } \\
\text { selbstzutragende Kosten und } \\
\text { Notwendigkeit von } \\
\text { Untersuchungen }\end{array}$ & $\begin{array}{l}\text { Aushang im Wartezimmer, } \\
\text { Vordruck für Kostenübernahme } \\
\text { (Int. } 7,26)\end{array}$ \\
\hline \multirow[t]{2}{*}{ Ausnahmen } & - Nur bei sozialer Indikation & $\begin{array}{l}\text { Abrechnung prinzipiell aus } \\
\text { formalen Gründen notwendig: } \\
\text { „Ein nicht quittiertes Attest ist ein } \\
\text { nicht ausgestelltes Attest.“ (Int. } \\
\text { 25) }\end{array}$ \\
\hline & $\begin{array}{l}\text { Konsequente Abrechnung trotz } \\
\text { geringer Vergütung }\end{array}$ & $\begin{array}{l}\text { Einheitliche Abrechnungspraxis } \\
\text { fördert Bewusstsein für } \\
\text { Dokumentencharakter und damit } \\
\text { verbundene Verantwortung (Int. } \\
\text { 26) }\end{array}$ \\
\hline \multirow[t]{3}{*}{ Anfragen Dritter } & $\begin{array}{l}\text { - Bewusstsein für Aufwand } \\
\text { fördern }\end{array}$ & \multirow{2}{*}{$\begin{array}{l}\text { Vordruck für Schule mit Hinweis } \\
\text { auf begrenzte Ressourcen und } \\
\text { Bitte um Kostenübernahme (Int. } \\
\text { 5) }\end{array}$} \\
\hline & $\begin{array}{l}\text { - Kostenübernahmeantrag an } \\
\text { anfragende Institution statt } \\
\text { Patienten senden }\end{array}$ & \\
\hline & $\begin{array}{l}\text { - Unzureichende } \\
\text { Vergütungsangebote mit } \\
\text { Kostenübernahmeantrag } \\
\text { beantworten }\end{array}$ & $\begin{array}{l}\text { Vordruck für } \\
\text { Krankenkassenanfragen (Int. 6) }\end{array}$ \\
\hline Untersuchungen & $\begin{array}{l}\text { - Können im Falle einer regulären } \\
\text { Untersuchung als } \\
\text { Kasselenleistung abgerechnet } \\
\text { werden }\end{array}$ & Check-Up Untersuchung (Int. 15) \\
\hline
\end{tabular}




\section{Einstellung und Umgang}

Die folgende Tabelle bietet eine Übersicht zum Umgang mit Schwierigkeiten bei der Attestausstellung und die Einstellung zur Standardisierung.

Tabelle 2: Systematische Übersicht über die Einstellung ausgewählter Interviewpartner zur Standardisierung von Attesten im Hinblick auf Unzufriedenheit mit der aktuellen Situation, Wunsch nach Veränderung, Offenheit für Verbesserungsvorschläge und

\section{Lösungsorientierung}

\section{Unzufriedenheit mit der aktuellen Situation}

\begin{tabular}{|c|c|c|}
\hline \multirow[t]{2}{*}{ Verantwortung } & $\begin{array}{l}\text { - Kann nur soweit übernommen } \\
\text { werden, wie Untersuchungen } \\
\text { eine Bestätigung zulassen }\end{array}$ & $\begin{array}{l}\text { Anamnese und Untersuchungen } \\
\text { schließen nicht alle } \\
\text { Unwägbarkeiten aus (Int. 1) }\end{array}$ \\
\hline & $\begin{array}{l}\text { - Keine verbindlichen } \\
\text { Untersuchungen für Atteste } \\
\text { festgelegt }\end{array}$ & $\begin{array}{l}\text { Anfrage sollte Checkliste zu } \\
\text { notwendigen Untersuchungen } \\
\text { beinhalten (Int. 1) }\end{array}$ \\
\hline \multirow[t]{3}{*}{ Abrechnung } & $\begin{array}{l}\text { - Mangelndes Verständnis für } \\
\text { Abrechnung / Aufwand auf } \\
\text { Seiten des Patienten }\end{array}$ & $\begin{array}{l}\text { „Ist doch nur eine } \\
\text { Unterschrift“ (Int.26) }\end{array}$ \\
\hline & $\begin{array}{l}\text { - Patient erwartet Abrechnung } \\
\text { der Untersuchungen als } \\
\text { Kassenleistung }\end{array}$ & $\begin{array}{l}\text { Patient erwartet Attest als } \\
\text { „Serviceleistung“ (Int. 1) }\end{array}$ \\
\hline & $\begin{array}{l}\text { - Aufwand der Abrechnung und } \\
\text { Rechtfertigung steht nicht im } \\
\text { Verhältnis zur Vergütung }\end{array}$ & $\begin{array}{l}\text { Abrechnung ist „lohnt sich gar } \\
\text { nicht“ (Int. 11) }\end{array}$ \\
\hline \multirow[t]{3}{*}{ Zeitaufwand } & $\begin{array}{l}\text { - Überproportional hoher } \\
\text { Zeitaufwand im Vergleich zu } \\
\text { Vergütung und medizinischem } \\
\text { Ergebnis }\end{array}$ & $\begin{array}{l}\text { Nicht nur der Zeitaufwand an sich, } \\
\text { auch der „mangelnde } \\
\text { medizinische Sinn“ mancher } \\
\text { Anfragen ist eine Belastung (Int. } \\
\text { 27) }\end{array}$ \\
\hline & $\begin{array}{l}\text { - Übermäßig hoher Zeitaufwand } \\
\text { für Verwaltungstätigkeit }\end{array}$ & $\begin{array}{l}\text { „Im Praxisalltag zählt jede Minute“ } \\
\text { (Int. 1) }\end{array}$ \\
\hline & - Unnötige / mehrfache Anfragen & $\begin{array}{l}\text { „Bürokratie ohne Sinn und } \\
\text { Verstand“ (Int. 27) }\end{array}$ \\
\hline \multirow[t]{2}{*}{ Definition } & - Unklare Anfragen & $\begin{array}{l}\text { Häufig werden „unrealistische } \\
\text { Bestätigungen“ gefordert (Int. 26) }\end{array}$ \\
\hline & $\begin{array}{l}\text { - Entscheidungsgrundlage nicht } \\
\text { vorgegeben }\end{array}$ & $\begin{array}{l}\text { vgl. Verantwortung: Anfrage sollte } \\
\text { Checkliste zu notwendigen } \\
\text { Untersuchungen beinhalten (Int. } \\
\text { 1) }\end{array}$ \\
\hline
\end{tabular}




\begin{tabular}{|l|l|l|}
\hline & $\begin{array}{l}\text { - Zeitliche Begrenzung der } \\
\text { Bestätigung nicht berücksichtigt }\end{array}$ & $\begin{array}{l}\text { Bestätigungen haben ein } \\
\text { „unbekanntes Ablaufdatum“ (Int. } \\
21)\end{array}$ \\
\hline Medizinische Relevanz & $\begin{array}{l}\text { - Anfragen können oft auch vom } \\
\text { Arzt nicht sicher beantwortet } \\
\text { werden }\end{array}$ & $\begin{array}{l}\text { „Wenn mir ein Schüler erzählt, } \\
\text { dass er Kopfschmerzen hat, kann } \\
\text { ich das genauso wenig } \\
\text { überprüfen wie sein Lehrer“ (Int. } \\
27)\end{array}$ \\
\hline $\begin{array}{ll}\text { Missbrauch der Autorität des } \\
\text { Arztes }\end{array}$ & $\begin{array}{l}\text { „Häufig ist die Bestätigung weder } \\
\text { die Aufgabe des Arztes noch } \\
\text { erfordert sie dessen } \\
\text { Ausbildung.“ (Int. 27) }\end{array}$ \\
\hline
\end{tabular}

\section{Wunsch nach Veränderung}

\begin{tabular}{|l|l|l|}
\hline Orientierungshilfen & $\begin{array}{l}\text { - Grundaufbau für eigene } \\
\text { Vorlagen }\end{array}$ & $\begin{array}{l}\text { Grundgerüst mit Standard } \\
\text { Angaben und Raum für } \\
\text { Ergänzungen (Int. 2) }\end{array}$ \\
\hline $\begin{array}{l}\text { - Standardisierte Vorlagen für } \\
\text { kleine Atteste }\end{array}$ & $\begin{array}{l}\text { Krankschreibungen, } \\
\text { Tauglichkeitsuntersuchungen (Int. } \\
1,3)\end{array}$ \\
\hline $\begin{array}{l}\text { - genaue Vorgaben für } \\
\text { Untersuchungen }\end{array}$ & $\begin{array}{l}\text { Checkliste / klare Angaben im } \\
\text { Attest (Int.1, 11) }\end{array}$ \\
\hline $\begin{array}{l}\text { Einheitliche Vergütung } \\
\text { Bbrechnung }\end{array}$ & $\begin{array}{l}\text { Bessere Regelungen für } \\
\text { Abrechnung mit einfachen } \\
\text { Pauschalen (Int. 26) }\end{array}$ \\
\hline Patienteneinsicht fördern & $\begin{array}{l}\text { "Man sollte Erziehungsarbeit am } \\
\text { Patienten leisten.“ (Int. 4) }\end{array}$ \\
\hline
\end{tabular}

\section{Offenheit für Standardisierung}

\begin{tabular}{|c|c|c|}
\hline \multirow[t]{7}{*}{ Vorteile } & - Verbindlichkeit & \multirow{6}{*}{$\begin{array}{l}\text { Klare Vorgaben würden die } \\
\text { Qualität des Attests und die damit } \\
\text { verbundenen Aspekte wie } \\
\text { Verbindlichkeit, Vollständigkeit } \\
\text { und Verantwortung, die sich } \\
\text { gegenseitig bedingen, } \\
\text { sicherstellen und Zeit sparen. (Int. } \\
1,4)\end{array}$} \\
\hline & - Verlässlichkeit & \\
\hline & - Strukturierung & \\
\hline & - Zeitersparnis & \\
\hline & - Vollständigkeit & \\
\hline & - Entlastung von Verantwortung & \\
\hline & $\begin{array}{l}\text { - Entlastung für die } \\
\text { Praxisangestellten }\end{array}$ & $\begin{array}{l}\text { „Angestellte müsste nicht jedes } \\
\text { diktierte Attest tippen“ (Int. 1) }\end{array}$ \\
\hline \multirow[t]{2}{*}{ Vorbehalte } & $\begin{array}{l}\text { - Einschränkung durch starre } \\
\text { Vorgaben }\end{array}$ & $\begin{array}{l}\text { „Vorsicht vor Überregulation (Int. } \\
11 \text { ) }\end{array}$ \\
\hline & $\begin{array}{l}\text { - Mehraufwand durch aufwendige } \\
\text { Formulare }\end{array}$ & $\begin{array}{l}\text { Umständliche Formulare könnten } \\
\text { zusätzliche Arbeit verursachen } \\
\text { (Int. 2, 11) }\end{array}$ \\
\hline
\end{tabular}




\section{Offenheit für Standardisierung}

\section{- Mangelnde Individualisierbarkeit Vordrucke lassen meist keinen Raum zur Hervorhebung von Besonderheiten (Int. 2) \\ - Vorlagen nicht für jedes Attest / jede Situation möglich \\ „Präzise Begründung von komplexen Sachverhalten oft nicht durch Vorlage ersetzbar" (Int. 2)}

\begin{tabular}{|c|c|c|}
\hline Lösungsorientierung & & \\
\hline \multirow[t]{3}{*}{$\begin{array}{l}\text { Verantwortung und } \\
\text { Vollständigkeit }\end{array}$} & $\begin{array}{l}\text { - Wenn Bestätigung Kompetenz } \\
\text { übersteigt: Überweisung zu } \\
\text { anderem Facharzt }\end{array}$ & $\begin{array}{l}\text { Im Zweifel immer einen Teilbefund } \\
\text { vom zuständigen Facharzt } \\
\text { einholen (Int. 1) }\end{array}$ \\
\hline & - Eigene Definition angeben & $\begin{array}{l}\text { „Aus internistischer Sicht“ (Int. } \\
\text { 21), ,zum Zeitpunkt der } \\
\text { Untersuchung“ (Int. 27) }\end{array}$ \\
\hline & - Patienten einbinden & $\begin{array}{l}\text { „Wenn der Patient dazu in der } \\
\text { Lage ist, lasse ich ihn auch selbst } \\
\text { formulieren und überprüfe den } \\
\text { Inhalt“ (Int. 28) }\end{array}$ \\
\hline \multirow[t]{7}{*}{ Vorgehen \& Vorlagen } & - Praxisangestellte einarbeiten & $\begin{array}{l}\text { Angestellte kann Atteste erstellen } \\
\text { und vom Arzt überprüfen lassen } \\
\text { (Int. 28) }\end{array}$ \\
\hline & $\begin{array}{l}\text { - Systematische } \\
\text { Vorgehensweisen erarbeiten }\end{array}$ & $\begin{array}{l}\text { Es hat sich gelohnt, Zeit in die } \\
\text { Erarbeitung einer systematischen } \\
\text { Vorgehensweisee zu investieren. } \\
\text { (Int. 28) }\end{array}$ \\
\hline & $\begin{array}{l}\text { - Standard Argumente für frei } \\
\text { formulierte Atteste erarbeiten }\end{array}$ & $\begin{array}{l}\text { Textbausteine für bestimmte } \\
\text { Atteste lassen sich immer wieder } \\
\text { verwenden (Int. 28) }\end{array}$ \\
\hline & - Eigene Vordrucke & \multirow{2}{*}{$\begin{array}{l}\text { Sparen „mentalen Aufwand“ } \\
\text { (Int. 4) }\end{array}$} \\
\hline & - Eigene Checklisten & \\
\hline & - Textbausteine & $\begin{array}{l}\text { Ermöglichen mehr Flexibilität } \\
\text { (Int. 28) }\end{array}$ \\
\hline & $\begin{array}{l}\text { - Praxissoftware mit } \\
\text { Hilfestellungen }\end{array}$ & $\begin{array}{l}\text { Schnittstelle mit } \\
\text { Textverarbeitungsprogramm } \\
\text { (Int. 28) }\end{array}$ \\
\hline
\end{tabular}




\section{Aktuelle Vorgehensweisen}

Um die aktuelle Situation im Hinblick auf Vorgehensweisen zu erfassen, wurden die Punkte Vorlagen, Abrechnung, Untersuchungen und Zuständigkeit standardmäßig abgefragt. Eine Auflistung der Ergebnisse mit absoluten Häufigkeiten in tabellarischer Form findet sich im Anhang. Die Ergebnisse werden in der folgenden Zusammenfassung mit den wichtigsten Ergebnissen zu den Themen Problem- und Verbesserungspotential und Einstellung und Umgang wiedergegeben.

\section{Zusammenfassung}

\section{Definition, Vollständigkeit und Verantwortung}

Als grundlegende Herausforderung bei der Attestausstellung wurde die unklare Definition des zu bestätigenden Inhalts genannt. Weiterhin ist nicht festgelegt, wie diese Aussage zu verifizieren ist, ob also die Einschätzung des Arztes nach Anamnese oder körperlicher Untersuchung ausreicht oder beispielsweise eine Laboruntersuchung notwendig ist (Ertl $L$ 2016). Dies führt gelegentlich dazu, dass ein Attest nicht als vollständig angesehen wird und neu ausgestellt werden muss. Bei unklaren Anforderungen besteht die Möglichkeit, den Patienten eine Vorlage von der anfragenden Stelle einholen zu lassen. Geeignete Patienten können das Attest auch selbst formulieren, wenn der Arzt die Richtigkeit der Angaben überprüft und bestätigt.

Im Zusammenhang mit dem Problem der Verifizierung einer Aussage wurde darauf hingewiesen, dass ein Patient, der ein bestimmtes Ziel verfolgt, sich in einem gewissen Rahmen verstellen kann (Ertl L 2016).

Außerdem kann sich der Zustand der Patienten beispielsweise bei Krankschreibungen vom Zeitpunkt der Attestausstellung bis zur Einreichung bereits wieder geändert haben. Durch unklare Definitionen werden häufig unrealistische Bestätigungen gefordert, für die der Arzt nicht die Verantwortung übernehmen kann. Der Arzt kann nur die Verantwortung dafür übernehmen, auf Grundlage vorgegebener Untersuchungen klar und realistisch definierte Aussagen zu bestätigen (Ertl L 2016). 


\section{Medizinische Relevanz}

Laut Aussagen der befragten Ärzte sei nicht immer der Zeitaufwand alleine problematisch, sondern die Tatsache, dass dieser nicht im Verhältnis zum Inhalt stehe.

Dies wird deutlich am Beispiel einer einfachen Krankschreibung, deren Ausstellung alleine kaum Zeit in Anspruch nimmt. Der Patient nimmt allerdings einen ganzen Termin in Anspruch, meist ohne dass sein Besuch eine medizinische Konsequenz hat. Oft scheint der Arztbesuch auch deshalb aus medizinischer Sicht unnötig, weil der Arzt einen Zustand wie Schuluntauglichkeit auf Grund einer Krankheit ebenso wenig verifizieren kann wie der Lehrer des Patienten. In diesem Fall entstehen unnötige Kosten für Patienten, Krankenkasse und Arzt (Ertl L 2016).

\section{Datenschutz und Schweigepflicht}

Als Schwierigkeit im Hinblick auf Datenschutz und Schweigepflicht wurden insbesondere solche Atteste genannt, die eine explizite medizinische Begründung fordern. In diesem Fall ist darauf zu achten, nur Symptome und keine Diagnosen zu nennen. Werden diese ausdrücklich verlangt, ist die Diagnose so zu wählen, dass sie den Patienten nicht brandmarkt (Ertl L 2016).

Anfragen von Institutionen, die sich ohne Umweg über den Patienten direkt an den Arzt richten, stellen für die befragten Ärzte kein großes Problem dar. Die befragten Ärzte weisen in der Regel die anfragende Stelle daraufhin, dass eine Schweigepflichtsentbindung vom Patienten vorliegen muss, bevor das entsprechende Formular bearbeitet werden kann (Ertl L 2016).

\section{Vorlagen für Atteste und Untersuchungen}

Die meisten Ärzte verwenden bereits Vorlagen, insbesondere für kleinere Atteste.

Bestehende Formulare wurden meist selbst und teilweise mit Hilfe von Vorlagen aus dem Internet erstellt.

Trotz dieser bestehenden Formulare würde ein Großteil der Ärzte gerne standardisierte Vorlagen verwenden (Ertl L 2016). 
Die Ärzte, die sich keine Verbesserung durch Vorlagen erhoffen, gaben an, bereits größtenteils mit selbst erarbeiteten Vorlagen zu arbeiten. Dabei werden meist Vordrucke verwendet, in einem Fall wird die Formulierung durch digitale Textbausteine erleichtert und die Patientendaten automatisch mit Hilfe einer Praxis Software eingefügt. Mit diesem System kann in der Regel die Praxisangestellte die passenden Bausteine zusammenfügen, sodass der Arzt den Inhalt lediglich ergänzen und überprüfen muss.

Es wurde mehrfach darauf hingewiesen, dass komplexe Sachverhalte oft nicht durch eine starre Vorlage vermittelbar sind. Aus diesem Grund müssen die Atteste individualisierbar sein. Außerdem ist die Anpassungsmöglichkeit nach Ermessen des Arztes wichtig, um keinen Mehraufwand durch einen zu umfangreichen Fragen- oder Untersuchungskatalog zu verursachen. Die meisten Interviewpartner bestätigten, den Umfang der Untersuchung an den Patienten anzupassen. Bei einem jungen, dem Arzt lange bekannten Patienten besteht weniger Abklärungsbedarf als bei einem älteren Patienten, der sich zum ersten Mal vorstellt. Deshalb sollten die passenden Untersuchungen wählbar sein und die Möglichkeit für einen freien Kommentar bestehen. Um die Vollständigkeit zu gewährleisten, empfiehlt sich die Verwendung einer Checkliste für Untersuchungen, aus welcher der Situation angemessene Vorgehen ausgewählt werden können (Ertl L 2016). Mit einem Vermerk der durchgeführten Untersuchungen im Attest lässt sich der Inhalt relativieren. Wenn beispielsweise der Befund "frei von ansteckenden Krankheiten“ anamnestisch erhoben wurde, übernimmt der Arzt die Verantwortung für diese Feststellung, soweit sie ohne Untersuchungen möglich ist (Ertl L 2016).

In Bezug auf umfangreiche Vorlagen wurden auch die bestehenden Vorlagen von Institutionen wie den Krankenkassen zum Vergleich herangezogen. Mehr Übersichtlichkeit und Systematik und eine Digitalisierung der Vorlagen wurden von mehreren Ärzten als Verbesserungswünsche genannt.

\section{Abrechnung}

Im Gespräch wurde deutlich, dass zwischen den verschiedenen Praxen und auch innerhalb einer Praxis keine einheitliche Vorgehensweise verfolgt wird (Ertl L 2016). Nur wenige Gesprächspartner stellen prinzipiell jedes Attest in Rechnung, die meisten machen 
Ausnahmen bei sozialer Indikation, beispielsweise bei Schülern oder finanziell schlechter gestellten Patienten.

Als Begründung für die uneinheitliche Abrechnungspraxis gaben einige Ärzte an, bei den Patienten auf Unverständnis zu stoßen (Ertl L 2016). Ein Arzt gab an, in geeigneten Fällen erforderliche Untersuchungen mit einem regulären Check Up zu verbinden, sodass die Kosten von der Krankenkasse getragen werden.

Die Ärzte, die kein Problem mit der Einsicht der Patienten angaben, klären den Patienten in der Regel vorher über die zu erwartenden Kosten auf und weisen darauf hin, dass ein Attest nach der Berufsordnung für Ärzte honorarpflichtig ist (Ertl L 2016).

Im Zusammenhang mit der Abrechnung wurden häufig Anfragen von Institutionen wie privaten Krankenkassen angesprochen, die regelhaft unzureichende Vergütung anbieten. Dem begegnen die meisten Ärzte, indem sie die Kostenzusage für einen angemessenen Betrag erbitten, bis zu deren Vorliegen sie die Anfrage nicht bearbeiten (Ertl L 2016). Einige Ärzte gaben an, hierfür bereits einen Vordruck zu verwenden.

Gelegentlich wurde eine weitere Strategie angesprochen, um eine systematische Abrechnung zu gewährleisten und Attestanfragen zu verringern. Einige Ärzte gaben an, bei übermäßig häufigen Anfragen von Schulen zu Krankschreibungen oder Sporttauglichkeitsbescheinigungen direkt mit der Leitung Kontakt aufzunehmen und sie darüber zu informieren, dass sie Atteste erst nach Zusage der Kostenübernahme durch die Schule bearbeiten werden. Dies führe meist zu einem deutlichen Rückgang der Attestanfragen.

\section{Zeitaufwand + Attestarten}

Die Frage, welche Attestart im Alltag des Interviewpartners am meisten Raum einnimmt und welche am meisten Schwierigkeiten bereitet, konnte größtenteils nicht eindeutig beantwortet werden. Wenn eine Attestart genannt wurde, waren es Renten- und RehaAnträge oder Anfragen von Reiserücktrittsversicherungen, jeweils wegen des großen Umfangs der Formulare. 


\subsection{Attestbeispiele}

Insgesamt wurden 56 Beispielatteste für 15 verschiedene Attestkategorien von 10 Ärzten eingereicht. Eine Übersicht über die Verteilung der eingereichten Atteste auf verschiedene Kategorien findet sich im Anhang. 


\section{Diskussion}

\subsection{Interpretation der Ergebnisse}

Durch die Befragung konnte ein erster Überblick über die aktuelle Praxis der Ausstellung von Attesten in der hausärztlichen Versorgung gegeben werden. Dabei zeigten die Teilnehmer großes Interesse an einer Vereinheitlichung. Sowohl beim Prozess der Attestausstellung als auch bei der Abrechnung zeigen sich deutliche Unterschiede in den Vorgehensweisen. Diese Unterschiede spiegeln sich auch in den Zahlen zu Vergütung und Zeitaufwand wider.

Die Attestausstellung ist in der hausärztlichen Praxis als erster Anlaufstelle ein Alltagsgeschehen, das die Ärzte in erster Linie als Verwaltungsaufwand wahrnehmen. Die Teilnehmer stimmen zu, dass eine Standardisierung Vorteile für den Arbeitsablauf und die Qualität des Attests haben könnte.

Mit Rücksicht auf Datenschutzbestimmungen und Schweigepflicht ist die Berechtigung jeder Anfrage zu prüfen. Um die Grenzen der eigenen Verantwortung nicht zu überschreiten sind Atteste möglichst präzise zu formulieren (Ertl L 2016). In der Untersuchung konnten die häufigsten Attestanfragen identifiziert werden. Teilweise werden bereits Vordrucke verwendet. Die ermittelten Zahlen belegen, dass dadurch wertvolle Zeit gespart werden kann. Auch bei der Abrechnung kommt kein einheitliches Vorgehen zur Anwendung. Aus verschiedenen Gründen wird häufig auf die Vergütung verzichtet (Ertl L 2016).

Die Diskussion richtet sich nach den Themenkomplexen im Fragebogen. Die Ergebnisse des Fragebogens werden durch die entsprechenden Vorgehensweisen, Kritikpunkte und Verbesserungsvorschläge aus den Expertengesprächen ergänzt.

\section{Bedeutung der Attestausstellung im Praxisalltag}

Der Hausarzt ist die erste Anlaufstelle für die meisten Attestwünsche. Gegebenenfalls lässt er auffällige Befunde beim entsprechenden Facharzt abklären. Alle im Gespräch befragten Ärzte gaben an, letztendlich das Attest auszustellen, auch wenn ein Teilbefund von einem 
anderen Arzt angefordert wurde. Diese überwiegende Zuständigkeit belegt die Relevanz der Attestausstellung in der allgemeinärztlichen Praxis.

Die meisten Ärzte nehmen die Ausstellung von Attesten vorwiegend als Routinetätigkeit und Pflicht wahr. Als fürsorgliche Handlung im Sinne des Patienten wird sie deutlich seltener empfunden - also nicht als ärztliche Aufgabe im engeren Sinne. Auch zur Patientenakquisition als wirtschaftliche Notwendigkeit trägt sie kaum bei. Deshalb ist es wünschenswert, den bürokratischen Aufwand zu minimieren und die Zeit in die Behandlung des Patienten zu investieren.

Auch im Gespräch wurde deutlich, dass die Attestausstellung nicht als ärztliche Aufgabe und deshalb als Belastung empfunden wird. Dabei steht oft das Missverhältnis zwischen Zeitaufwand und medizinischer Relevanz im Vordergrund. Letztendlich nimmt der Patient zum Beispiel für eine Krankschreibung einen regulären Besprechungstermin ein, meist ohne dass der Besuch eine medizinische Konsequenz hat. Das Missverhältnis zwischen Zeitaufwand und Ergebnis sollte möglichst vermindert werden.

Oft scheint der Arztbesuch auch deshalb aus medizinischer Sicht unnötig, weil der Arzt einen Zustand wie Schuluntauglichkeit auf Grund einer Krankheit ebenso wenig verifizieren kann wie der Lehrer des Patienten: „Wenn mir ein Schüler erzählt, dass er Kopfschmerzen hat, kann ich das genauso wenig überprüfen wie sein Lehrer" (Int. 27) (Ertl L 2016). Bei einer Krankheit, die per se keinen Besuch beim Arzt in seiner medizinischen Funktion erfordert, wird die Autorität des Arztes zu Unrecht in Anspruch genommen: „Häufig ist die Bestätigung weder die Aufgabe des Arztes noch erfordert sie dessen Ausbildung“ (Int. 27).

Durch diese „Bürokratie ohne Sinn und Verstand“ (Int. 27) entstehen unnötige Kosten für Patienten, Krankenkasse und Arzt (Ertl L 2016).

\section{Argumente für die Standardisierung}

Der Zeitersparnis wurde mit Abstand am meisten Bedeutung beigemessen (97,1\%) (Ertl L 2016). Zusätzlich könnten Vorlagen dazu beitragen, die Vollständigkeit sicherzustellen, eine angemessene Honorierung zu legitimieren (85,5\%) und eine einheitliche Form innerhalb der Praxis zu wahren (80,2\%). Auf diese Aspekte soll im Folgenden genauer eingegangen werden. 
Weniger Bedeutung wurde den Annahmen beigemessen, dass durch Vorlagen die Achtung der Datenschutzbestimmungen (70,3\%) und die Entbindung von der Schweigepflicht $(65,1 \%)$ gewährleistet werden könnte oder dass der Arzt von Verantwortung entlastet werden könnte $(58,1 \%)$.

\section{Datenschutz und Schweigepflicht}

Eine mögliche Erklärung dafür ist die Tatsache, dass Schweigepflicht und Datenschutzbestimmungen im Alltag weniger Probleme bereiten, da bereits Lösungsstrategien vorhanden sind.

Da der Patient das Attest oft selbst beantragt, ist die Schweigepflichtsentbindung oft impliziert. Ist dies nicht der Fall, empfiehlt es sich, der entsprechenden Institution einen vorgefertigten Hinweis auf die Notwendigkeit einer Schweigepflichtsentbindung zukommen zu lassen, bevor das Attest bearbeitet wird (Ertl L 2016).

Problempotential sehen die Ärzte bei Attesten, die eine explizite medizinische Begründung fordern. Es empfiehlt sich, wenn möglich nur Symptome zu benennen. Wird ausdrücklich eine Diagnose verlangt, wie beispielsweise oft bei Prüfungsunfähigkeitsbestätigungen für Universitäten, sollte der Arzt „die Diagnose so wählen, dass sie für den Patienten nicht stigmatisierend ist" (Int.14) (Ertl L 2016).

Im Listserver Allgemeinmedizin wurde diese Problematik ausführlich diskutiert. Es ist schwer genug, wenn man eine Diagnose „in Ziffern pressen“ muss (Flintrop, Korzilius 2012, S.2). Mit Rücksicht auf Nachteile, die dem Patienten zu Unrecht aus einer Diagnose entstehen können, wie der Ausschluss einer Beförderung oder Ablehnung einer Versicherung (Bullerkotte 2015), werden häufig Ausweichdiagnosen aus dem Bereich der R- und Z-Diagnosen (aus den ICD-10 Kapiteln XVIII „Symptome und abnorme klinische und Laborbefunde, die anderenorts nicht klassifiziert sind“ und Kapitel XXI „Faktoren, die den Gesundheitszustand beeinflussen und zur Inanspruchnahme des Gesundheitswesens führen“ (DIMDI 2016)) gewählt. Dadurch soll nicht die Wahrheit verschleiert, sondern sorgfältig differenziert werden. Ist ein Patient beispielsweise nach einem belastenden Ereignis psychisch verstimmt, liegt es nahe, den Zustand vorerst als Kontaktanlass oder Anpassungsstörung einzuordnen statt als depressive Störung oder eine andere FDiagnose (aus dem Kapitel V „Psychische und Verhaltensstörungen“), die ähnlich interpretiert werden kann. 
Im Listserver warnt ein Arzt aufgrund persönlicher Erfahrung vor leichtfertigen Diagnosen. Nachdem ein Hausarzt beim Erstkontakt eine Hypertonie in die Patientenakte eingetragen hatte, nur „um irgendwas drin zu haben“, stieß der 27-jährige auf große Schwierigkeiten beim Abschluss einer Lebensversicherung (Pascha 2008).

Bittet allerdings ein Patient mit bekanntem Nikotinabusus sowie Diabetes und Herzinfarkt in der Familienanamnese kurz vor Abschluss einer Lebensversicherung darum, Risikofaktoren im Antrag zu verschweigen (Sonntag 2008), ist die Grenze der Rücksichtnahme überschritten.

Dass auf eventuelle Konsequenzen für den Patienten Rücksicht genommen wird soll nicht heißen, dass bekannte Diagnosen bewusst verschleiert werden dürfen, um dem Patienten Vorteile zu verschaffen.

\section{Verantwortung}

Dass dem Argument „von Verantwortung entlasten“ weniger Bedeutung beigemessen wurde, liegt möglicherweise an den verschiedenen Auslegungsmöglichkeiten der Aussage. Selbstverständlich kann eine Vorlage die Verantwortung, die der Arzt übernimmt, indem er den Inhalt des Attests bestätigt, nicht vermindern.

Eine Standardisierung kann nur von der Verantwortung entlasten, alle genannten Aspekte der Attestausstellung (wie Vollständigkeit und Schweigepflichtsentbindung) zu beachten und den Umfang der Verantwortung durch genaue Angaben einzugrenzen. So können weit gefasste Definitionen wie „frei von ansteckenden Krankheiten“ (Int.1) oder „tauglich“ (Int. 16) durch die Entscheidungsgrundlage relativiert werden (Ertl L 2016).

Der Zusatz „anamnestisch“ impliziert beispielsweise, dass die Feststellung auf den nicht objektivierbaren Angaben des Patienten beruht, wobei zu beachten ist, dass dieser eventuell ein bestimmtes Ziel mit dem Attest verfolgt: „Wie weit kann sich der Patient verstellen?“ (Int. 16). „Auf Grundlage der körperlichen Untersuchung“ räumt ein, dass eine symptomlose Infektion vorliegen kann. Der Arzt übernimmt die Verantwortung für diese Feststellung also nur, soweit sie ohne weitere Untersuchungen feststellbar ist. „Zum Zeitpunkt der Untersuchung“ weist auf den flüchtigen Charakter der Zustandsbeschreibung und damit auf ein „unbekanntes Ablaufdatum“ (Int. 21) der Bestätigung hin. 
Das folgende Beispiel aus der Praxis illustriert die Absurdität mancher Forderungen: Ein Attest für das Sportstudium, das bestätigen soll, dass nach eingehender Untersuchung Allgemeinzustand, Bewegungsapparat, Herz-Kreislauf-, Atem- und Nervensystem sowie Hör- und Sehfähigkeit ohne pathologischen Befund seien. Diese Bestätigung sollte auch „den Zustand unter Belastung und den Aufenthalt im chlorierten Wasser" umfassen. Die mit diesem Attest konfrontierte Ärztin erklärte daraufhin, dass „mindestens ein BelastungsEKG, eine Spirometrie (mit Belastung?), Hör- und Sehtest, körperliche Untersuchung, und das ganze mit Belastung und im Schwimmbad... fällig wären“ (Frohnes 2015). Letztendlich bestätigte sie, dass anamnestisch keine Einschränkungen der Sporttauglichkeit vorliegen. Diese Formulierungen helfen beim Umgang mit „Forderungen unrealistischer Bestätigungen“ (Int.16).

\section{Bestehende Vorgehensweisen}

\section{Eigene Vordrucke}

Um die Relevanz einer Standardisierung zu beurteilen, wurden bestehende Vorgehensweisen bei Attesten, die ohne Vorlage angefordert werden, eruiert. Insgesamt muss knapp die Hälfte der Atteste eigens formuliert werden. Über die Hälfte der Ärzte formuliert in diesem Fall jedes Mal frei (Ertl L 2016). Demzufolge besteht deutliches Potential für die Verbreitung von Vorlagen.

Bei den Praxen, die eigene Vordrucke verwenden, füllt nur in jedem 10. Fall die MTA das Attest aus. Durch gut durchdachte Formulare wäre es denkbar, diese Zahl zu erhöhen und mit Hilfe der MTA mehr Zeit zu sparen. Es reicht für den Arzt aus, den Inhalt zu überprüfen, gegebenenfalls zu ergänzen und mit seiner Unterschrift zu bestätigen. Im Gespräch wurde deutlich, dass die meisten Ärzte das beschriebene Verbesserungspotential bereits erkannt haben. Auch wenn sie teilweise bereits eigene Vorlagen verwenden, würden viele eine Standardisierung begrüßen.

Ein Großteil der befragten Ärzte verwenden bereits Vorlagen, überwiegend für Atteste mit geringem Aufwand wie Schulatteste, etwa ein Drittel verwendet umfangreichere Vorlagen. Trotz dieser bestehenden Formulare würden die meisten Ärzte gerne standardisierte Vorlagen verwenden (Ertl L 2016). Die befragten Ärzte, die sich keine Verbesserung durch Vorlagen erhoffen, geben an, bereits größtenteils mit selbst erarbeiteten Vorlagen zu arbeiten. 


\section{Textbausteine}

Ein Arzt gab an, vorwiegend mit vorgefertigten digitalen Textbausteinen zu arbeiten. Die Bausteine werden je nach Situation ausgewählt und in einem Textverarbeitungssystem zusammengefügt und in ein Grundgerüst für Atteste mit Praxisdaten integriert.

Ärzte wünschen sich, mehr Verwaltungsarbeiten delegieren zu können (Statistisches Bundesamt 2015). Mit dem eben beschriebenen System kann in der Regel die MTA die passenden Bausteine zusammenfügen, sodass der Arzt den Inhalt lediglich ergänzen und überprüfen muss.

Diese Vorgehensweise spart wie die gängigen Vorlagen Zeit und Aufwand, weil nicht jedes Mal neu formuliert werden muss. Sie spart mentalen Aufwand, da Argumente für ähnliche Situationen aus vorangegangenen Attesten wiederverwendet werden können. Durch die individuelle Anpassbarkeit und die Möglichkeit, zusätzliche Informationen in freier Form hinzuzufügen wird mehr Flexibilität gewährleistet.

Kleine Atteste wie Krankschreibungen für die Schule, die nicht individualisiert sein müssen, lassen sich sicherlich nach wie vor besser mit einer einfachen Vorlage bewältigen. Die Arbeit mit Textbausteinen scheint besonders für die Atteste geeignet, deren komplexen Inhalt man nicht in eine starre Vorlage zwängen kann, also für Atteste mit gutachterlichem Charakter oder Atteste mit Untersuchungen (Ertl L 2016). Durch die freie Anpassungsmöglichkeit wird verhindert, dass zusätzlicher Aufwand durch einen umfangreichen Fragen- und Untersuchungskatalog verursacht werden.

\section{Intelligenter Einsatz von Praxissoftware}

Für die Arbeit mit Textbausteinen ist eine Praxissoftware mit Schnittstelle für ein Textverarbeitungssystem von Vorteil. So lassen sich in das Attest Patientendetails, relevante Diagnosen und Untersuchungsergebnisse automatisch aus der Patientenkartei in der Praxis Software übernehmen (Ertl L 2016).

Die Softwareanbieter von Praxis-EDV haben den Bedarf für Hilfestellungen bei der Attesterstellung bereits erkannt. Einige Systeme bieten die Möglichkeit, Attestvorlagen anzulegen, in welche die Patientendaten und Untersuchungsergebnisse automatisch eingefügt werden. Außerdem gibt es Textbausteine, die durch Anklicken in die entsprechenden Felder eingesetzt werden können. Die vorgeschlagenen Textbausteine 
können individuell für jedes Eingabefeld angepasst werden. Bereits erstellte Atteste können übernommen und aktualisiert werden. Auch aus Attesten in Papierform können Formulierungen eingepflegt werden.

Diese Neuerungen können den Arbeitsablauf erleichtern und sind bei der Auswahl einer Praxissoftware nicht außer Acht zu lassen.

Auch das Projekt Mehr Zeit für Behandlung hat das Potential von Hilfestellungen in der Praxissoftware erkannt und eine Handlungsempfehlung zu „Erläuterungstexten für die vereinbarten Vordrucke in der Praxissoftware" (Statistisches Bundesamt 2015) erstellt, die in den folgenden Abschnitten erläutert wird.

Prozessanalysen von Krankenkassen belegen, dass ein Großteil ihrer Anfragen durch fehlerhaft oder unvollständig ausgefüllte Formulare verursacht wird. Dementsprechend wichtig ist es, Formulare und Verordnungen, aber auch Ausfüllhilfen, so zu gestalten, dass Rückfragen von Krankenkassen gar nicht erst notwendig werden.

Bisher waren Vordruckerläuterungen uneinheitlich und oft umständlich formuliert. Mit der Praxissoftware sollen kurze Erläuterungen in Form einer Hilfefunktion durch einfaches Anklicken des entsprechenden Feldes schnell zugänglich gemacht werden. So könnte das Ausfüllen verbessert und gleichzeitig Zeit gespart werden.

Auch bei der Vereinheitlichung von Formularen wurde die Möglichkeit einer zukünftigen

Digitalisierung bereits bedacht.

\section{Untersuchungs-Checklisten}

Aus den Expertengesprächen ging hervor, dass es wichtig ist, auch Flexibilität in der Auswahl der Untersuchungen zu wahren. 27 der 31 Ärzte im Expertengespräch gaben an, den Umfang der Untersuchungen an den Patienten anzupassen. Bei einem jungen, dem Arzt lange bekannten Patienten besteht weniger Abklärungsbedarf als bei einem älteren Patienten, der sich zum ersten Mal vorstellt. Deshalb sollten die passenden Untersuchungen wählbar sein und die Möglichkeit für einen freien Kommentar sichergestellt werden. Um die Vollständigkeit zu gewährleisten, empfiehlt es sich, eine Checkliste für Untersuchungen einzusetzen. Mit einem strukturierten Untersuchungskatalog kann sichergestellt werden, dass unnötige Untersuchungen bewusst weggelassen und notwendigen Untersuchungen nicht versehentlich ausgelassen werden (Ertl L 2016). 


\section{Einbindung des Patienten}

Im Hinblick auf Vollständigkeit wurde im Expertengespräch mehrmals empfohlen, bei unklaren Anfragen den Patienten darum zu bitten, genaue Angaben oder eine Vorlage von der anfragenden Stelle einzuholen. Durch diese Maßnahme lässt es sich oft vermeiden, dass man ein Attest ein zweites Mal ausstellen muss, weil sich im Nachhinein herausstellt, dass Angaben fehlen. Zwei Ärzte gaben weiterhin an, geeignete Patienten das Attest selbst formulieren zu lassen. Solange der Arzt die Richtigkeit des Inhalts anschließend überprüft, ist diese Strategie legitim und in geeigneten Fällen sicherlich eine effiziente Lösung.

\section{Art der Attestanfragen}

Natürlich kann nicht für jede Situation eine Vorlage erstellt werden. Es ist davon auszugehen, dass der Bedarf an Vorlagen von der Häufigkeit der Attestanfragen bestimmt wird. Am häufigsten werden Atteste für gesetzliche Krankenkassen (21,88\%), Versorgungsämter (15,26\%), Schulen (14,61\%), Rentenversicherungen und Reha-Anträge $(12,93 \%)$ und Arbeitgeber (10,15\%) angefordert. Bei der Erarbeitung von Vorlagen scheint es deshalb sinnvoll, sich auf die genannten Gruppen zu konzentrieren.

Auch im Projekt Mehr Zeit für Behandlung wurden einige Informationspflichten als besonders bürokratieintensiv identifiziert, die unter die Rubrik Atteste fallen (Statistisches Bundesamt 2015, S.44). Diese werden in den folgenden Abschnitten zusammengefasst. Insgesamt wurden 42 Informationspflichten identifiziert, die jeweils mehr als 10 Millionen Euro kosten und 94,6\% des gesamten bürokratischen Zeitaufwands verursachen. Dabei lagen „Auskünfte an Krankenkassen und MDK auf vereinbarten Vordrucken“ und „formfrei“ auf Rang 5 respektive 10, „Bescheinigung der Arbeitsunfähigkeit auf Muster 1 und Prüfung genauer Umstände und Ausnahmetatbestände“ auf Rang 7 und „Erteilung von Auskünften und Bescheinigungen gegenüber den Krankenkassen im Rahmen der Erfüllung der ihnen obliegenden Aufgaben“ auf Rang 16.

3,33 Mrd. Euro der Kosten aus Informationspflichten werden durch die Regelungen der gemeinsamen Selbstverwaltung, 0,99 Mrd. Euro durch die Gesetzgebung des Bundes verursacht. Dabei ist zu berücksichtigen, dass Informationspflichten auf Ebene der 
gemeinsamen Selbstverwaltung meist eine gesetzliche Grundlage haben. Dennoch besteht Handlungsspielraum auf dieser Ebene, um die Regelungen zu vereinfachen. Das Projekt zeigt, dass sich die Entwicklung verkürzter, verständlicher Verfahren und Formulare auszahlt. Die Ergebnisse dieser Bemühungen sollen im Folgenden beleuchtet werden.

\section{Gesetzliche Krankenkassen}

Die Anfragen von gesetzlichen Krankenkassen, die in der Befragung den größten Teil der Attestanfragen ausmachen, werden in der Regel in Form von vorgefertigten Formularen der Krankenkasse angefordert. Im Expertengespräch machten mehrere Ärzte darauf aufmerksam, dass eine einheitliche Form durch bessere Vertrautheit des Arztes mit dem Formular sowie eine Digitalisierung dieser Anfragen viel Zeit sparen könnten.

Zu diesem Schluss ist man auch im Projekt Mehr Zeit für Behandlung gekommen, sodass bereits konkrete Strategien zur Umsetzung erarbeitet wurden. Es soll eine regelmäßig Überprüfung stattfinden, um sicherzustellen, dass Vordrucke nur aktuell notwendige Informationen erfragen und möglichst leicht verständlich sind. Durch eine Überarbeitung des Musters 52 in einen einheitlichen Vordruck soll die bessere Verständlichkeit und bürokratieärmere Umsetzung ermöglicht werden. Diese Verfahrensumstellung könnte zudem eine digitale Übermittlung der Informationen begünstigen, was unter bestimmten Voraussetzungen ebenfalls zum Bürokratieabbau beitragen kann (Statistisches Bundesamt 2015).

Ein großer Teil der Anfragen wird ohne Vordrucke angefordert. Wie für jedes Attest, das ohne Vorlage angefragt wird, lohnt es sich, Eigeninitiative zu ergreifen und sich eine einheitliche Vorgehensweise anzueignen.

Bei Anfragen, die nicht vom Patienten selbst kommen, ist dabei besonders auf den Datenschutz zu achten.

Auf den Anfrageformularen, die die Partner der Bundesmantelverträge vereinbart haben, dürfen Kassen Informationen direkt in der Praxis einholen. In den Expertengesprächen wird berichtet, dass die Formulare häufig ergänzt oder verändert werden oder Informationen ohne Formular angefragt werden. Bei Abweichungen von der vereinbarten Form ist die Rechtmäßigkeit der Anfrage in Frage zu stellen (Kassenärztliche Vereinigung Bayerns 2014b). Krankenkassen haben die Auflage, Informationen zu Diagnose und 
Behandlung beim Medizinischen Dienst der Krankenversicherung (MDK) einzuholen. Im Zweifelsfall auf diese Vorgabe hinzuweisen.

\section{Anfragen vom Versorgungsamt}

Auch für Anfragen vom Versorgungsamt liegen keine Vereinfachungsstrategien vor. Hier lässt sich ebenfalls die Anwendung einer eigenen Vorgehensweise zur Vereinfachung empfehlen. Da die Problematik meist komplex ist und eine individuelle Argumentation erfordern, sind Textbausteine in diesem Fall wohl besser geeignet als starre Vorlagen.

\section{Schule}

Durch einen einfachen Vordruck lässt sich der zeitliche Aufwand für ein einzelnes Schulattest auf ein Minimum reduzieren. Der Aufwand wird in diesem Fall durch die Menge der Attestanfragen verursacht und lässt sich nur begrenzen, indem ein vernünftiger Umgang mit Attestforderungen der Schule gefördert wird.

Wie bereits diskutiert ist der Arzt häufig genauso wenig im Stande, die Schulunfähigkeit zu bestätigen bzw. auszuschließen, dass diese nur vorgetäuscht wird. Diese Anfragen reduzieren unrechtmäßigerweise die Zeit, die der Versorgung der Patienten zusteht. Einer der Ärzte, die im Rahmen der Umfrage Beispielatteste zur Verfügung gestellt haben, weist darauf mit einem Vordruck hin, wenn sich Attestanfragen einer Schule häufen. Diese einfache Maßnahme ist ein guter Ansatz, um die Einsicht auf Seiten der Antragsteller zu fördern.

\section{Rentenversicherung \& Reha-Anträge}

Zur Verschlankung des Verfahrens zur Verordnung von medizinischer Rehabilitation wurde im Projekt des Normenkontrollrats beschlossen, dass das Muster 60 („Einleitung von Leistungen zur Rehabilitation oder alternativen Angeboten") entfallen soll (Statistisches Bundesamt 2015). Bei Unsicherheit über den zuständigen Kostenträger kann vorerst nur Teil A des Antragsformulars Muster 61 („Verordnung von medizinischer Rehabilitation“), 
ansonsten kann direkt die Verordnung der medizinischen Rehabilitation ausgestellt und an die Krankenkasse versandt werden.

\section{Bescheinigung von Arbeitsunfähigkeit}

Zur Zeit der Umfrage zum Thema „Atteste“ wurde zur Bescheinigung von

Arbeitsunfähigkeit während der Zeit des Anspruchs auf Entgeltfortzahlung durch den Arbeitgeber das Muster 1 verwendet. Die Arbeitsunfähigkeit für die Zahlung von Krankengeld wurde durch die Krankenkassen auf Muster 17 bescheinigt. Im Projektbericht wird die Problematik dieser Regelung und die Verbesserungsstrategie dargelegt: Da das Muster 17 keinen Durchschlag für den Arbeitgeber enthielt, forderten diese häufig eine datenschutzrechtlich bedenkliche Kopie oder zusätzlich ein Muster 1. Eine solche Doppeldokumentation ist rechtlich zwar nicht vorgesehen, wurde jedoch oft durchgeführt, um der Belastungen durch den Arbeitgeber zu vermeiden. Dadurch erhöhte sich die bürokratische Belastung unnötigerweise.

Die Kassenärztliche Bundesvereinigung und der Spitzenverband Bund der Krankenkassen haben ein Muster entworfen, mit dem der bisherige Auszahlschein in die Bescheinigung der Arbeitsunfähigkeit auf Muster 1 integriert wird. Durch eine bundesweite Vereinheitlichung des Musters soll eine IT-gestützte Ausfüllung ermöglicht werden. Zusätzlich wird geprüft, inwieweit die elektronische Datenübermittlung mit Rücksicht auf Datenschutzaspekte möglich ist.

Durch einen regelmäßigen Durchschlag für den Arbeitgeber soll die Doppeldokumentation vorweggenommen werden. Ein weiterer Durchschlag für den Patienten soll die rechtlichen Anforderungen an den Arbeitsunfähigkeitsnachweis erklären, um eine lückenlose Bescheinigung sicherzustellen (Statistisches Bundesamt 2015).

Es wird interessant sein zu sehen, wie sich die Veränderungen durch das neue Muster 1, das seit Beginn 2016 verwendet wird, auf den bürokratischen Aufwand im Praxisalltag tatsächlich auswirken. 


\section{Effizienzsteigerung durch Vorlagen}

\section{Zeitaufwand pro Attest}

97\% der Ärzte gaben an, sich von einer Standardisierung eine Zeitersparnis zu erhoffen. Um dies zu überprüfen, wurde der Zeitaufwand pro Attest für die verschiedenen Attestgruppen jeweils mit Vorlage und bei freier Formulierung verglichen. Es wird deutlich, dass bei jeder Gruppe die durchschnittliche Zeit durch die Verwendung von Vorlagen verringert wird (Ertl L 2016).

Bei aufwendigeren Attesten mit Untersuchungen oder gutachterlichem Charakter scheint deutliches Potential zur Zeitersparnis zu bestehen: Atteste mit Vorlage dauern hier 7 respektive 8 Minuten weniger. Der ohnehin geringe Aufwand bei kleineren Attesten lässt sich hingegen nur um etwa eine Minute reduzieren (Ertl L 2016).

\section{Wöchentlicher Zeitaufwand}

Die Zeitersparnis scheint in den zwei Gruppen der aufwendigeren Atteste also ähnlich zu sein. Der zeitliche Aufwand und die Fallzahl sind relevante Faktoren für die Höhe des bürokratischen Aufwands (Statistisches Bundesamt 2015). Im Alltag ist deshalb vor allem der Vergleich des gesamten Aufwands von Interesse, der sich aus dem Zeitaufwand pro Attest und der Zahl der angefragten Atteste ergibt.

Pro Woche werden in der Regel etwa 11 Atteste mit geringem Aufwand, 4 Atteste mit gutachterlichem Charakter und 3 Atteste mit Untersuchungen angefordert. Setzt man die Zahl der Anfragen ins Verhältnis mit der möglichen Zeitersparnis, ergibt sich insbesondere bei Attesten mit gutachterlichem Charakter ein deutliches Potential zur Effizienzsteigerung. Im Mittel könnte der Aufwand bei diesen um 36 Minuten pro Woche verringert werden. Bei Attesten mit Untersuchungen könnten 21 Minuten gespart werden, bei kleinen Attesten 20. Zusammengenommen ergibt sich daraus eine Ersparnis von mehr als einer Stunde pro Woche, was eine beachtliche Zahl ist, da „im Praxisalltag jede Minute zählt“ (Int. 1) (Ertl L 2016). 


\section{Abrechnung}

\section{Vorgehen}

Um die Attestausstellung in einen wirtschaftlichen Kontext zu setzen, wurden im Fragebogen die verschiedenen Vorgehensweisen bei der Abrechnung, sowie die erstatteten Beträge ermittelt. Die mangelnde Systematik in der Attestausstellung spiegelt sich auch in der Abrechnung wider. Im Gespräch wurde deutlich, dass zwischen den verschiedenen Praxen und auch innerhalb einer Praxis keine einheitliche Vorgehensweise verfolgt wird (Ertl L 2016).

Die wenigsten der befragten Ärzte stellen prinzipiell jedes Attest in Rechnung, die meisten machen Ausnahmen bei sozialer Indikation, beispielsweise bei Schülern oder finanziell schlechter gestellten Patienten. Laut Berufsordnung soll bei Honorarvereinbarungen die finanzielle Situation des Patienten berücksichtigt werden (Bundesärztekammer 2015). Insgesamt werden knapp 75\% der Atteste abgerechnet. Nur ein Drittel der Ärzte rechnet ausnahmslos ab (Ertl L 2016).

Etwa $40 \%$ der Ärzte gaben an, Atteste per Barzahlung abzurechnen, weitere $40 \%$ gaben an, eine Rechnung auszustellen. $20 \%$ wählen die Methode abhängig vom Einzelfall. Um die konsequente Abrechnung sicherzustellen scheint es sinnvoll, ein einheitliches System in der Praxis anzuwenden, wie zum Beispiel die Abrechnung per Barzahlung bis zu einem gewissen Grenzbetrag, respektive per Rechnung für höhere Beträge.

Die mangelnde Konsequenz bei der Abrechnung ist vermutlich einer der Gründe, warum Patienten sich uneinsichtig zeigen, wenn es um die Abrechnung von Attesten geht. Eine einheitlichere Abrechnungspraxis könnte dazu beitragen, die Patienteneinsicht zu fördern (Ertl L 2016). Ebenso könnte eine einheitliche Form der Atteste den Dokumentencharakter betonen und dadurch zu einer angemessenen Honorierung beitragen. Andersherum würde auch die Honorierung den Dokumentencharakter des Attests stützen: „Ein nicht quittiertes Attest ist ein nicht ausgestelltes Attest" (Int.25).

Als Begründung für die uneinheitliche Abrechnungspraxis wiederum gaben einige Ärzte an, bei den Patienten auf Unverständnis zu stoßen (Ertl L 2016). Die mangelnde Einsicht des Patienten und die mangelnde Konsequenz des Arztes bei der Abrechnung schienen sich also gegenseitig zu bedingen beziehungsweise zu verstärken. Für die geringe 
Vergütung wird die Rechtfertigung und der Abrechnungsaufwand ungern in Kauf genommen (Montes de Oca, 2013). Dennoch sollte sie eingefordert werden, da die Berufsordnung im Regelfall die Liquidierung von Attesten vorschreibt.

Weil der Patient vermutlich gewohnt ist, dass die Krankenkasse die Kosten des Arztbesuchs übernimmt, erwartet er das Attest als „Serviceleistung“ (Int. 1) und rechnet nicht damit, selbst für das Dokument und die damit verbundene Untersuchungen aufkommen zu müssen. Hier ist die Unterscheidung verschiedener Situationen wichtig (Pieritz 2010): Ist die Ausstellung des Attests der alleinige Anlass für Beratung und Untersuchung, können diese ebenfalls privat berechnet werden. Für einen regulären Arztbesuch kommt die Krankenkasse auf, wenn ein Attest als "Nebenprodukt" ausgestellt wird hat der Patient nur die Kosten dafür zu tragen. Werden nur Kopien der Befunde oder Behandlungsdokumentation erstellt, kann keine Gebühr nach der Gebührenordnung für Ärzte (GOÄ) erhoben werden.

Bundes- und Landesärztekammern stellen Poster zur Verfügung, welche die Patienten über diese Regelungen aufklären.

Ein Arzt gab im Gespräch an, in geeigneten Fällen erforderliche Untersuchungen mit einem Check Up zu verbinden, sodass die Kosten von der Krankenkasse getragen werden. Diese Verbindung mit einer regulären Untersuchung ist durchaus legitim und es bietet sich an, diese Gelegenheit nach Möglichkeit zu nutzen.

Die Ärzte, die kein Problem mit der Einsicht der Patienten angaben, klären den Patienten in der Regel vorher über die zu erwartenden selbstzutragenden Kosten auf, wie es in der Berufsordnung vorgesehen ist (Bundesärztekammer 2015). Es scheint ratsam, beispielsweise mit Hilfe eines Aushangs im Wartezimmer auf die Abrechnung von Attesten hinzuweisen, um die Patienteneinsicht zu fördern und ein einheitliches Vorgehen in der Praxis durchzusetzen. Geeignete Poster werden von Bundes- und Landesärztekammern zur Verfügung gestellt.

Dass die Rechtfertigungsnot vor dem Patienten viele Ärzte davon abhält, abzurechnen, scheint auch folgende Feststellung nahezulegen: Wenn dem Patienten aus dem Attest ein finanzieller Vorteil entsteht, stellen es in etwa doppelt so viele Ärzte in Rechnung. Wer mit dem Attest einen finanziellen Vorteil verfolgt, wird keine Einwände haben, die zielführende Bestätigung auch zu bezahlen. Für ein Attest für die Reiserücktrittsversicherung ist die Attestgebühr letztlich gut investiert, wenn der Patient dadurch die Gesamtkosten der gebuchten Reise zurückerstattet bekommt. 
Während aus Sicht des Patienten die Abrechnung oft nicht im Verhältnis zur Leistung des Arztes steht - „Ist doch nur eine Unterschrift“ (Int. 26) - steht aus Sicht des Arztes die Entschädigung häufig nicht im Verhältnis zum Aufwand.

Etwa zwei Drittel der Ärzte rechnen ausschließlich die vorgegebene Ziffer ab, die anderen addieren bei erheblichem Aufwand eine Zusatzgebühr. Die meisten Ärzte könnten also neben der konsequenteren Abrechnung von Attesten auch die Möglichkeit nutzen, den Betrag besser dem tatsächlichen Aufwand anzupassen.

\section{Vergütung}

Um die Effizienzsteigerung in einen wirtschaftlichen Kontext zu setzen, wurde der Betrag ermittelt, der für die verschiedenen Attestgruppen erstattet wird. Atteste mit Untersuchungen werden durchschnittlich mit $46 €$ vergütet, Atteste mit gutachterlichem Charakter mit $30 €$, Atteste mit geringem Aufwand mit $4 €$.

Um die Entschädigung mit dem Aufwand ins Verhältnis zu setzen und dieses Verhältnis beim Vorgehen „mit Vorlage“ und „ohne Vorlage“ vergleichbar zu machen, wurde ein fiktiver „Stundenlohn“ aus dem Betrag und der aufgewendeten Zeit berechnet (Ertl L 2016):

- Bei Attesten mit geringem Aufwand ließe sich der Stundenlohn durch die Verwendung von Vorlagen von $83 €$ auf $136 €$, also um $60 \%$, steigern.

- Bei Attesten mit gutachterlichem Charakter ließe sich der Stundenlohn von 74€ auf 104€, also um $41 \%$, steigern.

- Bei Attesten mit Untersuchungen ließe sich der Stundenlohn von $165 €$ auf $184 €$, also um $11 \%$, steigern.

Im Hinblick auf die Wirtschaftlichkeit bietet die Verwendung von Vorlagen bei allen Attestgruppen also eine deutliche Verbesserung (Ertl L 2016).

Einige Versicherungen bieten regelmäßig unzureichende Vergütung für das Ausstellen von Befundberichten und Gutachten (Ertl L 2016).

Die auf dem Justizvergütungs- und -entschädigungsgesetz (JVEG) basierenden Vergütungen für ärztliche Befundberichte und Gutachten wird von der 
Bundesärztekammer für zu niedrig gehalten (Golfier 2011). Die Möglichkeit der individuellen Vergütungsvereinbarung sollte dafür genutzt werden, eine bessere Honorierung zu erzielen.

Dennoch machen viele Versicherungen ein individualvertragliches Angebot, das weit unter der festgelegten Honorierung liegt. Der Arzt muss dieses Angebot nicht annehmen, da ihm die gesetzliche Vergütung zusteht.

Auch Anfragen von Krankenkasse werden häufig nicht korrekt honoriert. Vereinbarte Vordrucke der Krankenkassen sind an dem Aufdruck Muster X zu erkennen. Wenn die Krankenkasse darauf zusätzliche Fragen stellt oder ändert oder eine Anfrage ohne vereinbarten Vordruck stellt, kann der Arzt diese unbeantwortet an die Krankenkasse zurücksenden oder eine Kostenübernahmeerklärung einholen (Kassenärztliche Vereinigung Bayerns 2014b) (Ertl L 2016). Danach kann er privat abrechnen, wenn eine Einverständniserklärung des Patienten vorliegt. Die KVB stellt eine Musterantwort für diese Anfragen bereit.

Auch die Bitte um eine Kostenzusage bei anderen Institutionen, wie zum Beispiel Schulen, ist eine mögliche Strategie um die Vergütung sicherzustellen oder Attestanfragen zu verringern. Einige Ärzte gaben im Interview an, bei übermäßig häufigen Anfragen von Schulen direkt mit der Leitung Kontakt aufzunehmen. Dies führe meist zu einem deutlichen Rückgang der Attestanfragen. 


\subsection{Kritische Betrachtung der Datenerhebung und Auswertung}

\section{Kollektiv}

Nähere Informationen zum Ärzteprofil sind nicht bekannt. Bei der Auswertung fiel auf, dass Angaben zur Anzahl der Attestanfragen in einzelnen Praxen stark vom Mittelwert abweichen. Faktoren wie Patientenzahl und -zusammensetzung (Alter, soziale Lage), die Lage der Praxis (Stadt/Land) und besonderer Focus (z.B. Tauchmedizin) beeinflussen sicherlich Parameter wie Zahl und Art der Anfragen sowie Abrechnungspraktiken. Bei einer zukünftigen Umfrage wäre es sinnvoll, diese Punkte zu erfragen, um Abweichungen vor deren Hintergrund genauer betrachten zu können.

\section{Rücklaufquote}

Aufgrund der Verteilung über verschiedene Kanäle lässt sich keine Rücklaufquote ermitteln. Lehrärzte wurden direkt per Email kontaktiert, zusätzlich wurde der Link zum Onlinefragebogen im Listserver Allgemeinmedizin, mehreren Fachzeitschriften, deren Newsletter und Onlineauftritten veröffentlicht. Dadurch ist die Zahl der hausärztlich tätigen Ärzte, die die Bitte um Teilnahme an der Umfrage gelesen haben, leider nicht abschätzbar.

\section{Teilnahmebereitschaft}

Durch die hohe Teilnehmerzahl für den Fragebogen (172) führte die statistische Auswertung zu signifikanten Ergebnissen. Es ist zu beachten, dass die Teilnehmer wie beschrieben nicht nach Faktoren wie Patientenzahl oder Lage der Praxis selektioniert worden sind. Dies könnte die große Spannweite mancher Ergebnisse im Fragebogen erklären. Unter diesen Bedingungen lassen sich die Ergebnisse nicht eins zu eins auf die Grundgesamtheit übertragen, erlauben aber einen guten Überblick über die aktuelle Situation. 
Die 31 Gesprächsteilnehmer ermöglichten es, eine große Bandbreite von Ansichten zu beleuchten. Auch wenn die Zahl für eine statistische Auswertung zu gering ist, ließen sich bestimmte Trends erkennen.

Die Tatsache, dass sich so viele Ärzte Zeit für die Auseinandersetzung mit dem Thema genommen haben, spricht für eine gewisse Relevanz des Themas im Praxisalltag (Ertl L 2016). Möglicherweise haben sich vor allem Ärzte Zeit für den Fragebogen bzw. das Gespräch genommen, für die durch die Attestausstellung erheblicher Aufwand entsteht. Demzufolge könnten die ermittelten Werte für Zeitaufwand und Zahl der Anfragen höher liegen als erwartet. Die Vermutung liegt nahe, dass Ärzte eher an der Umfrage teilgenommen haben, wenn sie Verbesserungspotential sehen. Die Argumente für eine Standardisierung wurden deshalb möglicherweise öfters positiv bewertet als es in der Grundgesamtheit der Fall wäre.

\section{Anonymisierung}

Durch die Anonymisierung der Umfrage ist zu erwarten, dass alle Fragen ehrlich und ohne Einschränkungen beantwortet werden konnten. Auch wenn anzunehmen ist, dass dies in schriftlicher Form leichter ist als im Gespräch, äußerten die Befragten besonderes im offenen Gespräch Kritik. 


\section{Fragebogen}

\section{Fragebogendesign}

Die geschlossenen Fragen wurden vom Großteil der Teilnehmer eindeutig beantwortet und anschließend statistisch ausgewertet.

Die offenen Fragen wurden qualitativ ausgewertet und im Expertengespräch vertieft und liefern erstmalig einen Überblick über verschiedene Aspekte der Attestausstellung (Ertl L 2016). In einer weiteren Studie könnten diese Ergebnisse quantifiziert werden.

\section{Auswertung}

Es liegt in der Natur der Sache, dass Bewertungen von Empfinden und Bedeutung von Argumenten sich nur bis zu einem gewissen Grad objektivieren und messen lassen. Die im Fragebogen verwendete Skala sollte diese ins Verhältnis setzen und so bestmöglich beschreiben. Es ist anzumerken, dass sich hier nur eine Rangfolge von 1-4 ohne definierte Abstände bilden lässt. Beispielsweise bedeutet in Frage 5 („Wie wichtig sind für Sie folgende Argumente und sprechen damit für die Standardisierung von Attesten?") die Bewertung „sehr wichtig“ (Punkt 4 auf der Skala) natürlich nicht, dass ein Argument doppelt so wichtig ist wie bei der Bewertung „weniger wichtig“ (Punkt 2 auf der Skala). Dementsprechend wurden bei der Auswertung nur Häufigkeiten der Angaben angegeben und keine Mittelwerte berechnet.

\section{Formulierung}

Trotz mehrfacher Überarbeitung ist eine missverständliche Formulierung erst im Nachhinein aufgefallen. Die Frage 2.3 „Welcher Anteil wird frei formuliert?" hätte den Sachverhalt klarer mit „Welche Anteil wird ohne Vorlage angefragt und erfordert eine eigene Formulierung?" beschrieben. Dementsprechend wäre die Frage 2.4 „Wie werden frei formulierte Atteste erstellt?" mit „Wie werden Atteste formuliert, die ohne Vorlage angefragt werden?" präziser gewesen. 


\section{Gesprächsleitfaden \& Expertengespräche}

\section{Gesprächsleitfadendesign}

Mithilfe des Gesprächsleitfadens konnte ein teilstrukturiertes Interview mit offenen Fragen durchgeführt werden. Die geschlossenen Fragen im Fragebogen konnten so optimal ergänzt werden.

Die Form des teilstrukturierten Interviews gab Anhaltspunkte zur Gesprächsführung und ließ gleichzeitig Raum, auf Punkte, zu dem der Gesprächspartner besonders viel beizutragen hatte, genauer einzugehen.

Anders als im Fragebogen gab es keine Verständnisprobleme durch missverständliche Formulierungen, da Unklarheiten bei Fragen im Gespräch sofort beseitigt werden konnten. Andersherum konnten auch unklare Antworten direkt angesprochen und geklärt werden. Die Dauer des Gesprächs wurde bei der Rekrutierung auf 10-15 Minuten geschätzt. Letztendlich hing die Dauer jedes Gesprächs davon ab, wie ausführlich die Fragen beantwortet wurden. Das kürzeste Gespräch dauerte 6 Minuten, das längste 26, in der Regel wurde das ursprünglich geschätzte Zeitlimit eingehalten.

\section{Aufzeichnung}

Die Aufzeichnung der Gespräche ermöglichte einen schnelleren Ablauf und stellte sicher, dass kein Wort verloren ging. So konnte bei der schriftlichen Zusammenfassung das gesamte Gespräch noch einmal nachvollzogen werden.

\section{Fragestellung}

Die Beantwortung der meisten Fragen im Gesprächsleitfaden schien den Ärzten keinerlei Probleme zu bereiten. Durch die vorangegangene Bearbeitung des Fragebogens hatte sich der Gesprächsteilnehmer bereits mit der Problematik auseinandergesetzt. Dies war sicherlich eine gute Vorbereitung für das Interview, viele Teilnehmer äußerten bereits vor den konkreten Fragen konkrete Anregungen und Verbesserungsvorschläge. 
3 Fragen schienen für die meisten Ärzte nicht einfach zu beantworten, wie sich bei der Durchführung der Interviews herausstellte. Auf diese wurde in späteren Interviews verzichtet, um mehr Zeit auf die Vertiefung anderer Fragen verwenden zu können. Die Frage „Welche GOÄ Ziffern verwenden Sie für welche Atteste?" konnte selten eindeutig beantwortet werden. In der Regel wurden die Ziffern 70, 75 oder 76 zwar genannt, welche Ziffer wofür verwendet wird, konnte aus dem Stegreif nicht beantwortet werden. Dies liegt wahrscheinlich daran, dass die Abrechnung in den meisten Praxen nicht Aufgabe des Arztes ist.

Auch die Frage „Wie viel Zeit nehmen Atteste Ihrem Empfinden nach im Alltag ein? (Atteste mit Untersuchungen, gutachterlichem Charakter, kleine Atteste)" stellte sich im Verlauf als ungeeignet heraus. Die Bewertungen „Wenig / angemessen / übermäßig viel“ konnte von den meisten Gesprächsteilnehmern nicht eindeutig zugeordnet werden. Dies wurde meist damit begründet, dass die Einschätzung von Attest zu Attest innerhalb jeder Gruppe je nach Zweck und Vorlage variiert.

Die Frage „Überwiegt eine Art von Attesten im Hinblick auf Zeitaufwand / Problempotential?" wurde ebenfalls nur zögerlich beantwortet. Auch hier wurde argumentiert, dass die Einschätzung je nach Umfang der Anforderungen für Atteste der gleichen Gruppe stark variieren kann. 


\section{Zusammenfassung}

Atteste werden aus den verschiedensten Gründen angefordert. Dabei gibt es weder formale noch inhaltliche Standards. Eine Vereinheitlichung könnte Zeit sparen und die Qualität der Atteste verbessern.

Vor dem Hintergrund des steigenden Bedarfs von medizinischer Versorgung liegt es im dringenden Interesse aller Beteiligten, den Verwaltungsaufwand zu minimieren. Nur so bleibt dem hochqualifizierten Mediziner genügend Zeit, den Patienten optimal zu versorgen (Ertl L 2016).

Die vorliegende Arbeit soll einen Überblick über aktuelle Relevanz, Indikationen und Hindernisse der Attestausstellung in der hausärztlichen Praxis bieten. In diesem Zusammenhang sollte der Bedarf einer Standardisierung eruiert werden. Dazu wurden Zeitaufwand, Arbeitsbelastung und bestehende Vorgehensweisen bei der Attestausstellung beleuchtet (Ertl L 2016).

In einem anonymisierten Fragebogen mit offenen Fragen (für Durchschnittswerte mit vorgegebener Einheit), Multiple Choice Fragen und vierstufig skalierten Fragen wurden konkrete Zahlen zu Zeitaufwand, Anzahl und Art der angefragten Atteste erhoben. Der Fragebogen für hausärztlich tätige Allgemeinärzte und Internisten wurde in mehreren Fachzeitschriften, deren Online Portalen und Newsletter und im Listserver Allgemeinmedizin veröffentlicht und per Email an die Lehrärzte der Medizinsicher Fakultäten in München verteilt.

Die 172 Fragebögen wurden mit der Software SPSS mit Methoden der deskriptiven Statistik quantitativ ausgewertet (Ertl L 2016).

Zusätzlich wurden in 31 Experteninterviews Schwierigkeiten bei der Attestausstellung beleuchtet. Die Vor- und Nachteile von Vorlagen wurden herausgearbeitet und Verbesserungsvorschläge für diese erarbeitet. Die Auswertung der Ergebnisse richtet sich nach den Methoden der qualitativen Inhaltsanalyse nach Philip Mayring (Ertl L 2016). Mithilfe von anonymisierten Attestbeispielen wurde ein Überblick über bestehende Vorlagen gewonnen und Verbesserungsansätze erarbeitet.

Die rege Teilnahme an der Untersuchung belegt den Wunsch nach einer Vereinfachung. Als Grund steht die Effizienzsteigerung klar im Vordergrund. Dabei ist zu beachten, dass 
Vorlagen nicht durch starre Vorgaben zusätzlichen Aufwand verursachen oder die Möglichkeit einer individuellen Anpassung nehmen (Ertl L 2016).

Die Untersuchung zeigt, dass bei Attestausstellung und -abrechnung deutliche Unterschiede in den Vorgehensweisen bestehen (Ertl L 2016). Die Probleme, mit denen sich Ärzte häufig in der Praxis konfrontiert sehen, sind aber die gleichen (z.B. Datenschutz und Verantwortung).

Die ermittelten Zahlen belegen, dass Vorlagen wertvolle Zeit sparen können. Auf Grundlage dieser Feststellung und mit Rücksicht auf die angesprochenen Schwierigkeiten wurden Hilfestellungen erarbeitet. Um eine Vereinfachung sinnvoll zu gestalten, scheinen Vorlagen nur für einfache Atteste geeignet. Vor dem Hintergrund der Kritikpunkte sollten für aufwendigere Atteste dynamischere Vorgehensweisen wie eine Checkliste für empfehlenswerte Untersuchungen im Rahmen des Attests und Textbausteine verwendet werden, die eine individuelle Anpassung ermöglichen (Ertl L 2016).

Auch mehr Systematik in der Qualifikation der Ärzte für bestimmte Atteste (wie das Tauchtauglichskeitsattest) würde die Arbeit erleichtern und die Qualität und Vergleichbarkeit sicherstellen. Eine Untersuchung zum Thema dieser Zuständigkeit stünde sicherlich im Interesse der Standardisierung.

Der Abbau von Bürokratie auf politischer Ebene erfordert einen „langen Atem“ (Weißbrot, 2016). Die Vertreter der Selbstverwaltung arbeiten kontinuierlich an diesem komplexen Prozess. Um den Alltag in der eigenen Praxis zu erleichtern, ist Eigeninitiative gefragt. Die diskutierten Hilfestellungen bieten eine erste Orientierung. Die Untersuchung zeigt, dass es sich lohnt, Zeit in die Erarbeitung eines eigenen Systems investieren, um den Dieben der Zeit das Handwerk zu legen und auf lange Sicht mehr Zeit für die medizinische Versorgung des Patienten zu gewinnen (Ertl L 2016). 


\section{Literaturverzeichnis}

Barton AH, Lazarsfeld PF (1979). Einige Funktionen von qualitativer Analyse in der Sozialforschung. Stuttgart, Klett.

Birkner B, Dierks, ML (2009) Kompendium Q-M-A, Qualitätsmanagement in der ambulanten Versorgung. Köln.

Bonaparte N, zit. nach Gross S (2010). Süddeutsche Zeitung vom 17.05.2015. Im Internet: http://www.sueddeutsche.de/karriere/lebenskunst-ist-sich-vorzeitdiebenzu-schuetzen-1.596034 (zuletzt aufgerufen am: 01.04.16)

Bullerkotte A (2015). "35110." Allgmed Listserver. Im Internet: http://www.listserv.dfn.de/cgibin/wa?A2=ind150 \&L=ALLGMED-L\&P=R452271\&l=-3\&X

20A98544A447664750\&Y=livia.ertl

\%40hotmail.de (zuletzt aufgerufen am 10.03.16)

Bundesärztekammer (2015). (Muster-)Berufsordnung für die in Deutschland tätigen Ärztinnen und Ärzte: 4.

Carlsson L, Lannerstrom L, Wallman T, Holmstrom IK (2015). "General practitioners' perceptions of working with the certification of sickness absences following changes in the Swedish social security system: a qualitative focus-group study." BMC Fam Pract 16: 21.

Cooper RA, Getzen TE, McKee HJ and Laud P (2002). "Economic and demographic trends signal an impending physician shortage." Health Aff (Millwood) 21(1): 140-154.

De Boer W (2010). "Raus aus den Klompen." MDK Forum 4(4): 7-8.

Deutschen Institut für Medizinische Dokumentation und Information (DIMDI) (2016). ICD-10-GM Internationale statistische Klassifikation der Krankheiten und verwandter Gesundheitsprobleme, 10. Revision.

Dunstone DC, Reames HR (2001). "Physician satisfaction revisited." Soc Sci Med 52(6): 825-837. 
Ertl L, Sanftenberg S, Schelling J (2016) „Medical certificates and examinations in family doctor's office. Indications, barriers and relevance of standardization" MMW-Fortschr Med 158 (Suppl 6):1-4. Epub 2016 Dec8.

Flick U, Von Kardoff E, Steinke I (2000). Qualitative Forschung: Ein Handbuch. Hamburg, Merkens.

Flintrop J, Korzilius H (2012). "Bürokratie in Praxen und Krankenhäsuern: Vom Versuch, den Alltag in Ziffern zu pressen." Deutsches Ärzteblatt 109(13): A-634.

Frohnes S (2015). "Sport-Attest." Allgmed Listserver. Im Internet: http://www.listserv.dfn.de/ cgi-bin/wa?A2=ind1508\&L=ALLGMED-

$\underline{L} \& P=R 1415788 \& \mid=-3 \& X=2973 E 75$ A67B25121F1\&Y=livia.ertl\%40hotmail.de (zuletzt aufgerufen am 10.03.16)

Glaser B, Strauss A (2012). The discovery of grounded theory: Strategies for qualitative research. Chicago, Aldine Pub. Co.

Gläser J, Laudel G (2009). Experteninterviews und qualitative Inhaltsanalyse. Wiesbaden, VS Verlag für Sozialwissenschaften.

Golfier A (2011). "Honorierung von Befundberichten und Gutachten im Auftrag der Deutschen Rentenverischerung." Deutsches Ärzteblatt 108(34-35): A-1814.

Gross R, Tabenkin H, Brammli-Greenberg S (2007). "Factors affecting primary care physicians' perceptions of health system reform in Israel: professional autonomy versus organizational affiliation." Soc Sci Med 64(7): 1450-1462.

Herrmann WJ, Haarmann A, Bærheim A (2015). "Arbeitsunfähigkeitsregelungen als Faktor für Inanspruchnahme ärztlicher Versorgung in Deutschland." Zeitschrift für Evidenz, Fortbildung und Qualität im Gesundheitswesen 109(8): 552-559.

Hussey L, Money A, Gittins M and Agius R (2015). "Has the fit note reduced general practice sickness certification rates?" Occup Med (Lond) 65(3): 182-189. 
Kammer P (2015). Projektabschluss: „Mehr Zeit für Behandlung“ - So kann unnötige Bürokratie in Arzt- und Zahnarztpraxen abgebaut werden: 2. Im Internet: https:// www.normenkontrollrat.bund.de/Webs/NKR/Content/DE/Pressemitteilungen/ 2015-08-28 arztpraxenprojekt abschluss.html (zuletzt aufgerufen am 05.03.16)

Kassenärztliche Vereinigung Bayerns (2014a). Anlaufstelle für Bürokratie: Endbericht. München (S.7-25) Im Internet: https://www.kvb.de/fileadmin/kvb/dokumente/Praxis/ Praxisfuehrung/Projekte/KVB-Anlaufstelle-Buerokratieabbau-Endbericht-2014.pdf (zuletzt aufgerufen am 10.03.16)

Kassenärztliche Vereinigung Bayerns (2014b). Anfragen von gesetzlichen Krankenkassen (S.4). Im Internet: https://www.kvb.de/fileadmin/kvb/dokumente/Praxis/Infomaterial/ Praxisbetrieb/KVB-Broschuere-Anfragen-von-Krankenkassen.pdf (zuletzt aufgerufen am 15.03.16)

AT Kearney (2011) "Deutsches Gesundheitswesen auf dem Prüfstand - Kostenfalle Komplexität." Im Internet: https://www.atkearney.de/documents/856314/1214640/ Deutsches_Gesundheitssystem_auf_dem_Pruefstand.pdf/b5ca045a-128e-40cb-9763e73d35647279 (zuletzt aufgerufen am 15.03.16)

KPMG (2006). Standard-Kosten-Messung der bürokratischen Belastungen von Vertragsärzten im Bereich der Kassenärztlichen Vereinigung Westfalen-Lippe. Dortmund.

Kriedel T, Müller T, Boehnert H (2006). "600 Informationspflichten für den Vertragsarzt." Deutsches Ärzteblatt 103(51-52): A3450-A3454.

Kühbauch M (2010). "From sick note to fit note." MDK Forum (4): 9-10.

Kühbauch M, Weber A (2010). "Rückkehr in Raten." MDK Forum (4): 5-6.

Landrigan CP, Rothschild JM, Cronin JW, Kaushal R, Burdick E, Katz JT, Lilly CM, Stone PH, Lockley SW, Bates DW, Czeisler CA (2004). "Effect of reducing interns' work hours on serious medical errors in intensive care units." N Engl J Med 351(18): 1838-1848. 
Leech N, Onuwuegbuzie A (2010). "Guidelines for Conducting and Reporting Mixed Research in the Field of Counseling and Beyond." Journal of Counseling \& Development 88(3).

Levin S, Aronsky D, Hemphill R, Han J, Slagle J and France DJ (2007). "Shifting toward balance: measuring the distribution of workload among emergency physician teams." Ann Emerg Med 50(4): 419-423.

Linzer M, Visser MR, Oort FJ, Smets EM, McMurray JE, de Haes HC and Society of General Internal Medicine Career Satisfaction Study G (2001). "Predicting and preventing physician burnout: results from the United States and the Netherlands." Am J Med 111(2): 170-175.

Lockley SW, Cronin JW, Evans EE, Cade BE, Lee CJ, Landrigan CP, Rothschild JM, Katz JT, Lilly CM, Stone PH, Aeschbach D, Czeisler CA (2004). "Effect of reducing interns' weekly work hours on sleep and attentional failures." N Engl J Med 351(18): 1829-1837.

Mayring P (2002). Einführung in die Qualitative Sozialforschung. Weinheim, Beltz Verlag.

Mayring P (2010). Qualitative Inhaltsanalyse. Weinheim und Basel, Beltz.

Montes de Oca A (2013). "Erstellung von ärztlichen Attesten für den Kindergarten oder die Schule." Ärzteblatt Sachsen-Anhalt 8: 12.

Normenkontrollrat (2015). Mehr Zeit für Behandlung: Ergebnisse der Aufwandsermittlung. Im Internet: https://www.normenkontrollrat.bund.de/Webs/NKR/Content/DE/Download/ 2015-08-28 download-pm_arztpraxenprojekt_praesentationdestatis.pdf;jsessionid=0803CC5017532229 (zuetzt aufgerufen am 28.02.16)

OECD (2010). Sickness, Disability and Work: Breaking the Barriers, OECD Publishing. Im Internet: http://www.oecdbookshop.org/browse.asp?pid=title$\underline{\text { detail\&lang=en\&ds }=\& I S B=9789264088849}$ (zuletzt aufgerufen am 05.03.16)

Onwuegbuzie A, Leech N (2006). "Linking research questions to mixed methods data analysis 
procedures. The Qualitative Report." http://www.nova.edu/ssss/QR/QR/1-3/onwuegbuzie (12.03.16)

Onwuegbuzie AJ, Leech NL (2004). "Enhancing the interpretation of "significant" findings:

The role of mixed methods research." The Qualitative Report 9(4): 770-792.

Pascha G (2008). "Schweigepflicht, Dokumentation, Krankenkassen und

Versicherungen." Im Internet: http://www.listserv.dfn.de/cgi-bin/wa?

$\underline{A 2=i n d 0810 \& L=A L L G M E D-}$

$\underline{L} \& P=R 190757 \& 1=-3 \& X=3 F 08 D A 18 B F 302 A A 3 E 0 \& Y=$ livia.ert|\%40hotmail.de (zuletzt aufgerufen am 06.03.16)

Pieritz A (2010). "Attest ausstellen - wer bezahlt?" Deutsches Ärzteblatt(8): 360.

Shanafelt TD, Bradley KA, Wipf JE, Back AL (2002). "Burnout and self-reported patient care in an internal medicine residency program." Ann Intern Med 136(5): 358-367.

Shanafelt TD, Sloan JA, Habermann TM (2003). "The well-being of physicians." Am J Med 114(6): 513-519.

Shanafelt TD, West C, Zhao X, Novotny P, Kolars J, Habermann T, Sloan J (2005).

"Relationship between increased personal well-being and enhanced empathy among internal medicine residents." J Gen Intern Med 20(7): 559-564.

Sonntag B (2008). "Schweigepflicht, Dokumentation, Krankenkassen und Versicherungen." Im Internet: http://www.listserv.dfn.de/cgi-bin/wa?

A2=ind0810\&L=ALLGMED-

$\underline{L} \& \mathrm{P}=\mathrm{R} 190757 \& \mid=-3 \& \mathrm{X}=3 \mathrm{~F} 08 \mathrm{DA} 18 \mathrm{BF} 302 \mathrm{AA3E0} \& \mathrm{Y}=$ livia.ert|\%40hotmail.de (zuletzt aufgerufen am 28.02.16)

Statistisches Bundesamt, Gruppe Bürokratiekostenmessung, (2015). Mehr Zeit für Behandlung - Vereinfachung von Verfahren und Prozessen in Arzt- und Zahnarztpraxen: Abschlussbericht August 2015. (S.5-97). 
Strauss A, Corbin J (1990). Basics of qualitative research. Grounded theory procedures and techniques. Newbury Park, Sage.

Strauss A (1987). Qualitative Analysis for social scientists. Cambridge, University of Cambridge Press.

Torges B (2010). "Schweden: Ein Land geht an die Arbeit." MDK Forum 4(4): 11-12.

Virtanen P, Oksanen T, Kivimaki M, Virtanen M, Pentti J, Vahtera J (2008). "Work stress and health in primary health care physicians and hospital physicians." Occup Environ Med 65(5): 364-366.

Wallace JE, Lemaire JB, Ghali WA (2009). "Physician wellness: a missing quality indicator." Lancet 374(9703): 1714-1721.

Weißbrot J (2016). "Bürokratieabbau - Hartnäckigkeit zahlt sich aus." KVB Forum(3): 9.

Wenning M (2010) "Vergütung für Befundberichte und Gutachten durch Rentenversicherungsträger und deren Arbeitsgemeinschaften." Im Internet: http:// www.bundesaerztekammer.de/fileadmin/user upload/downloads/

BefundberichteGutachtenDRV.pdf (zuletzt aufgerufen am 18.02.16)

Williams ES, Skinner A (2003). "Outcomes of physician job satisfaction: a narrative review, implications and directions for future research." Health Care Manage Rev 28: 119-140.

Williams ES, Rondeau KV, Xiao Q, Francescutti LH (2007). "Heavy physician workloads: impact on physician attitudes and outcomes." Health Serv Manage Res 20(4): 261-269. 


\section{Anhang}

\subsection{Anschreiben, Fragebogen und Probandeninformation}

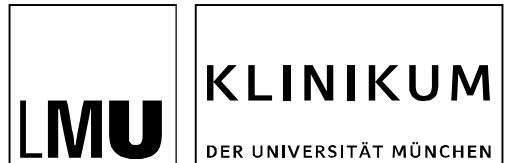

DER UNIVERSITÄT MÜNCHEN
CAMPUS INNENSTADT

INSTITUT FÜR ALLGEMEINMEDIZIN

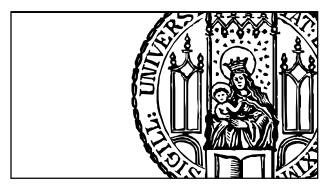

Klinikum der Universität München Institut für Allgemeinmedizin

Klinikum der Universitatt München
Pettenkoferstr. 8 a 80336 Münche

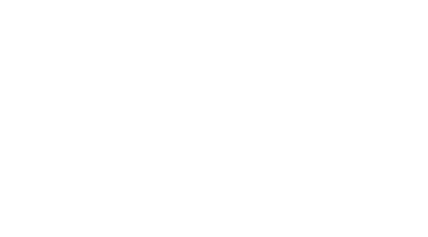

Ihr Zeichen:

Unser Zeichen:

Sehr geehrte Damen und Herren,

Die Anforderung diverser Atteste nimmt der hausärztlichen Praxis immer mehr Raum ein. Das Institut für Allgemeinmedizin der Ludwig-Maximilians Universität führt deshalb eine Studie durch, in der

Arbeitsbelastung, Zeitaufwand und Abrechnungsmöglichkeiten eruiert werden sollen. Anschließend sollen Standards erarbeitet werden, um das Ausstellen von Attesten zu vereinfachen.

Wir hoffen, mit dieser Studie Verbesserungen in Ihrem Interesse zu erreichen und wären Ihnen sehr dankbar, wenn Sie oder Ihre Assistenz den beiliegenden Fragebogen ausfüllen würden.

Dies wird nicht mehr als 2-5 Minuten in Anspruch nehmen.

Sie können den Fragebogen online unter folgendem Link aufrufen:

https://www.umfragen-am-klinikum.de/Atteste

Alternativ können Sie den Fragebogen im Anhang öffnen und per Email oder Post zurücksenden.

Wir würden wir uns besonders freuen, wenn Sie sich für ein ca. 20-minütiges Interview per Telefon oder in Ihrer Praxis bereit erklären würden oder anonymisierte Beispielatteste zur Verfügung stellen könnten. Bitte merken Sie im Fragebogen an, wenn Sie die Möglichkeit dafür sehen.

Bitte senden Sie uns eine kurze, formlose Absage, wenn Sie nicht an der Studie teilnehmen möchten. Wir sind Ihnen für Ihre aktive Unterstützung sehr dankbar und stehen jederzeit für Fragen zur Verfügung.

Mit freundlichen Grüßen,

Livia Ertl
Dr. rer. nat. Linda Sanftenberg Lehr- und

Forschungskoordinatorin

Institut für Allgemeinmedizin

Klinikum der Universität
Prof. Dr. med. Jörg Schelling Komm. Direktor

Institut für Allgemeinmedizin

Klinikum der Universität 
An alle Studienteilnehmer zur Forschungsarbeit

Sekretariat: Frau Wiltrud García Aznarán

Telefon +49 (0)89 $4400-53779$

Telefax +49(0)89 $4400-53520$

„Medizinische Atteste und Untersuchungen in der

allgemeinärztlichen Praxis - eine Übersicht und

empirische Befragung zu Indikationen, Hindernissen und Relevanz im hausärztlichen Alltag"

Email: allgemeinmedizin@med.uni-muenchen.de

Postanschrift:

Ihr Zeichen: Unser Zeichen: München, den

\section{Probandeninformation und Einwilligungserklärung}

\section{zur Studie}

„Medizinische Atteste und Untersuchungen in der allgemeinärztlichen Praxis - eine Übersicht und empirische Befragung zu Indikationen, Hindernissen und Relevanz im hausärztlichen Alltag"

Sehr geehrte Damen und Herren,

die Anforderung diverser Atteste nimmt der hausärztlichen Praxis immer mehr Raum ein. Das Institut für Allgemeinmedizin der Ludwig-Maximilians Universität führt deshalb eine Studie durch, in der Arbeitsbelastung, Zeitaufwand und Abrechnungsmöglichkeiten eruiert werden sollen.

Obwohl die eben genannten Punkte in der Praxis häufig diskutiert werden, liegen bis jetzt keine Daten zu diesem Thema vor.

Die Daten werden in Form von anonymisierten Fragebögen und Interviews erhoben. Die Interviews werden handschriftlich dokumentiert und nach Rücksprache mit Ihnen als Interviewpartnern auch aufgezeichnet. Die Tonaufnahmen werden nur mit Ihrem Einverständnis angefertigt, ausschließlich zur Auswertung des Interviews genutzt und anschließend gelöscht.

Außerdem werden anonymisierte Beispielatteste verwendet, um einen besseren Überblick über die aktuelle Handhabung der Attestanfragen zu bekommen. Anschließend sollen Standards erarbeitet werden, um das Ausstellen von Attesten zu vereinfachen. Für die Datenerhebung ist der Zeitraum bis November 2015 vorgesehen, deren Auswertung soll im April 2016 abgeschlossen werden. Über die Ergebnisse informieren wir Sie bei Interesse selbstverständlich gerne.

Die Teilnahme an der Studie erfolgt freiwillig und das Einverständnis zur Teilnahme an dem Forschungsvorhaben kann jederzeit und ohne Angabe von Gründen zurückgenommen werden, ohne dass Ihnen als Teilnehmer daraus Nachteile entstehen. Da sämtliche erhobenen Daten und persönlichen Aussagen anonymisiert und statistisch ausgewertet werden, ist eine Aussonderung von Fragebogen und/ oder Interview nach Abschluss der Datenerhebung jedoch nicht mehr möglich. Diese Umfrage soll helfen Aspekte zu benennen, die bei der Ausstellung von Attesten in der hausärztlichen Praxis durch eine Standardisierung verbessert werden könnten, um damit den hausärztliche Alltag zu entlasten.

Wir hoffen, mit dieser Studie Verbesserungen in Ihrem Interesse zu erreichen und wären Ihnen für Ihre Unterstützung sehr dankbar. Für Fragen stehen wir jederzeit zur Verfügung. 


\section{Einverständniserklärung}

Ich ..................... erkläre mich bereit, an einer Studie zum Thema „Medizinische Atteste und Untersuchungen in der allgemeinärztlichen Praxis - eine Übersicht und empirische Befragung zu Indikationen, Hindernissen und Relevanz im hausärztlichen Alltag" des Instituts für Allgemeinmedizin (Klinikum der Universität München) teilzunehmen. Meine Teilnahme erfolgt freiwillig und mir ist bekannt dass ich jederzeit und ohne Angabe von Gründen mein Einverständnis zur Teilnahme an dem Forschungsvorhaben zurücknehmen kann, ohne dass mir hieraus Nachteile entstehen. Da sämtliche erhobenen Daten und persönlichen Aussagen werden anonymisiert und statistisch ausgewertet werden, ist eine Aussonderung von Fragebogen und/ oder Interview nach Abschluss der Datenerhebung jedoch nicht mehr möglich.

Diese Umfrage soll helfen Aspekte zu benennen, die bei der Ausstellung von Attesten in der hausärztlichen Praxis durch eine Standardisierung verbessert werden könnten, um damit den hausärztliche Alltag zu entlasten.

Ort, Datum, Unterschrift

Herzlichen Dank für Ihre Unterstützung!

Prof. Dr. med Jörg Schelling

Leiter des Instituts für Allgemeinmedizin

Klinikum der Universität München 


\section{Medizinische Atteste und Untersuchungen in der allgemeinmedizinischen Praxis \\ Anonymisierter Fragebogen}

\section{Unter welche Kategorie fällt die Ausstellung von Attesten nach Ihrem persönlichen Empfinden? $(1=$ trifft nicht $z u, 2=$ trifft eher nicht $z u, 3=$ trifft eher $z u, 4=$ trifft voll $z u)$}

- Routinetätigkeit:

$1 \square 2 \square 3 \square 4 \square$

- Pflicht:

$1 \square 2 \square \quad 3 \square \quad 4 \square$

- Patientenakquisition:

$1 \square 2 \square \quad 3 \square 4 \square$

- Fürsorgliche Maßnahme zum Wohle meiner Patienten:

$1 \square 2 \square 3 \square 4 \square$

\section{Wie viel Zeit nimmt ein Attest durchschnittlich in Anspruch?}

\subsection{Vorgefertigte Formulare}

- Atteste mit Untersuchungen (z.B. Tauchattest, Versicherung):

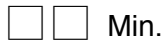

-Atteste mit gutachterlichem Charakter (z.B. Versorgungsamt, Rentenversicherung):

- Atteste mit geringerem Aufwand (z.B. Schulattest):

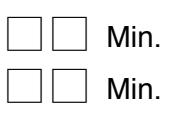

\subsection{Freie Form}

- Atteste mit Untersuchungen (z.B. Tauchattest, Versicherung):

- Atteste mit gutachterlichem Charakter (z.B. Versorgungsamt, Rentenversicherung):

\subsection{Wie werden frei formulierte Atteste erstellt?}

Eigener Vordruck, vom Arzt ausgefüllt

Eigener Vordruck, von der Praxisangestellten ausgefüllt

Attest wird jedes Mal frei formuliert 


\section{Wie viele Atteste stellen Sie pro Woche aus?}

- Atteste mit Untersuchungen (z.B. Tauchattest, Versicherung):

-Atteste mit gutachterlichem Charakter (z.B. Versorgungsamt, Rentenversicherung):

St.

-Atteste mit geringerem Aufwand (z.B. Schulattest):

St.

\section{Aus welchen Anteilen setzen sich die Anfragen zusammen?}

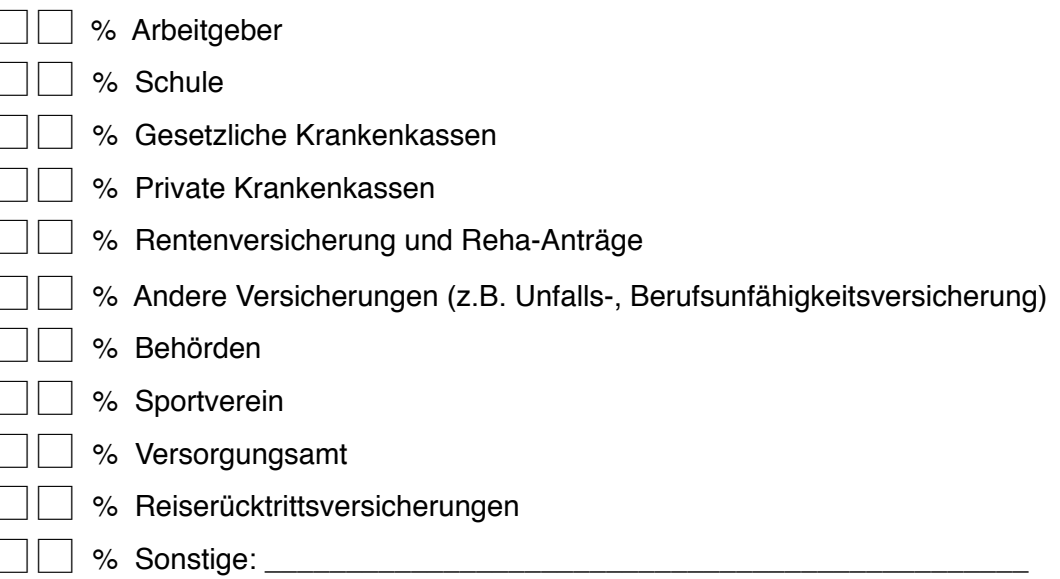

\section{Wie wichtig sind für Sie folgende Argumente und sprechen damit für eine} Standardisierung von Attesten?

(1 = unwichtig, 2 = weniger wichtig, 3 = eher wichtig, 4 = sehr wichtig)

Eine Standardisierung von Attesten könnte..

...durch einen Katalog

der notwendigen Untersuchungen die Vollständigkeit sicherstellen:

...von Verantwortung entlasten:

...die Achtung von Datenschutzbestimmungen sicherstellen :

...die Entbindung von der Schweigepflicht sicherstellen:

...Zeit sparen:

...zur Legitimation einer angemessen Honorierung beitragen:

$1 \square 2 \square 3 \square 4 \square$
$1 \square 2 \square 3 \square 4 \square$
$1 \square 2 \square 3 \square 4 \square$
$1 \square 2 \square 3 \square 4 \square$
$1 \square 2 \square 3 \square 4 \square$
$1 \square 2 \square 3 \square 4 \square$
$1 \square 2 \square 3 \square 4 \square$




\section{Attestabrechnung}

\subsection{Wie oft stellen Sie Atteste allgemein in Rechnung?}

$\square$ Nie $\square$ Selten $\square$ Häufig $\square$ Immer

Folgende Atteste stelle ich nie in Rechnung:

Folgende Atteste stelle ich immer in Rechnung:

$\%$ Anteil der abgerechneten Atteste

6.2 Wie oft stellen Sie Atteste in Rechnung, wenn der Patient primär einen finanziellen Vorteil durch das Attest erlangt?

$\square$ Nie $\square$ Selten $\square$ Häufig $\square$ Immer

6.3 Wie rechnen Sie ab?

Barzahlung

Gegen Rechnung

Gar nicht (bis zu einem gewissen Grenzbetrag:

(ab einem gewissen Grenzbetrag:
$€)$

$€)$

\subsection{Wie rechnen Sie bei vorgesehener Abrechnungsziffer ab?}

Ich rechne immer nur die Abrechnungsziffer ab

Bei erheblichem Mehraufwand wird eine zusätzliche Gebühr erhoben

\section{Welchen Betrag bekommen Sie durchschnittlich pro Attest erstattet?}

- Atteste mit Untersuchungen (z.B. Tauchattest, Versicherung):

- Atteste mit gutachterlichem Charakter (z.B. Versorgungsamt, Rentenversicherung):

-Atteste mit geringerem Aufwand (z.B. Schulattest):

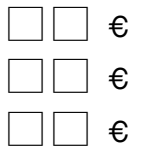




\section{Persönliches Gespräch}

Wenn Sie die Möglichkeit sehen, etwas mehr Zeit zu investieren, würden wir uns besonders freuen, wenn Sie sich für ein ca. 20-minütiges Interview, das streng anonym ausgewertet wird, per Telefon oder in Ihrer Praxis bereit erklären würden. Dürfen wir Sie diesbezüglich noch einmal kontaktieren, um einen Termin zu vereinbaren?

$\mathrm{Ja}$, per (Tel./Email)

Nein

\section{Attestbeispiele}

Um uns einen Überblick über die aktuellen Vorgehensweisen verschaffen zu können, suchen wir Vordrucke und Atteste in freier Form, wie Sie momentan in Ihrer Praxis ausgestellt werden. Wären Sie dazu bereit, uns streng anonymisierte Beispielatteste zur Verfügung zu stellen?

$\mathrm{Ja}$

Nein

\section{Einverständniserklärung}

Ich erkläre mich bereit, an einer Studie zum Thema „Medizinische Atteste und Untersuchungen in der allgemeinärztlichen Praxis - eine Übersicht und empirische Befragung zu Indikationen, Hindernissen und Relevanz im hausärztlichen Alltag“ des Instituts für Allgemeinmedizin (Klinikum der Universität München) teilzunehmen. Meine Teilnahme erfolgt freiwillig und mir ist bekannt dass ich jederzeit und ohne Angabe von Gründen mein Einverständnis zur Teilnahme an dem Forschungsvorhaben zurücknehmen kann, ohne dass mir hieraus Nachteile entstehen.

Sämtliche erhobenen Daten und persönlichen Aussagen werden anonymisiert und statistisch ausgewertet. Diese Umfrage soll helfen Aspekte zu benennen, die bei der Ausstellung von Attesten in der hausärztlichen Praxis durch eine Standardisierung verbessert werden könnten, um damit den hausärztliche Alltag zu entlasten.

Ort, Datum, Unterschrift

Gerne können Sie für Ihre Rückmeldung per Fax oder Post den beiliegenden Antwortbogen nutzen. Wenn Sie nicht teilnehmen möchten, wäre Ich Ihnen für eine kurze, formlose Absage dankbar.

Vielen Dank für Ihre Teilnahme! Livia Ertl 


\subsection{Tabellarische Auflistung der Ergebnisse des Fragebogens}

Tabelle 3.1 Bedeutung der Attestaustellung im Praxisalltag

\begin{tabular}{|l|r|r|}
\hline Bedeutung ,trifft (voll) zu“ & Häufigkeit $(\mathbf{n = 1 7 2 )}$ & Prozent \\
\hline Routinetätigkeit & 146 & 84,9 \\
\hline Pflicht & 135 & 78,5 \\
\hline Fürsorgliche Maßnahme & 105 & 61,0 \\
\hline Patientenakquisition & 17 & 9,9 \\
\hline
\end{tabular}

Tabelle 3.2 Argumente für die Standardisierung

\begin{tabular}{|l|r|r|}
\hline Argument „(sehr) wichtig“ $^{\prime \prime}$ & Häufigkeit (n=172) & Prozent \\
\hline Zeitersparnis & 167 & 97,1 \\
\hline Vollständigkeit & 148 & 86,0 \\
\hline Honorierung & 147 & 85,5 \\
\hline Einheitlichkeit & 138 & 80,2 \\
\hline Datenschutzbestimmungen & 121 & 70,3 \\
\hline Schweigepflicht & 112 & 65,1 \\
\hline Verantwortung & 100 & 58,1 \\
\hline
\end{tabular}

Tabelle 4.1 Bestehende Vorgehensweisen: Anteil eigens erstellter Atteste

\begin{tabular}{|c|c|c|c|c|c|}
\hline & $\begin{array}{l}\text { Mittelwert (in } \\
\% \text { ) }\end{array}$ & $\begin{array}{l}95 \% \mathrm{KI} \\
\text { (Unterer W) }\end{array}$ & $\begin{array}{l}95 \% \mathrm{KI} \\
\text { (Oberer W) }\end{array}$ & Minimum & Maximum \\
\hline Attest eigens erstellt & 45,85 & 42,50 & 49,21 & 0 & 100 \\
\hline
\end{tabular}

Tabelle 4.2 Bestehende Vorgehensweisen: Erstellung eigener Atteste

\begin{tabular}{|l|r|r|}
\hline Vorgehensweise & Häufigkeit (n=172) & Prozent \\
\hline Freie Formulierung & 96 & 55,8 \\
\hline $\begin{array}{l}\text { Eigener Vordruck: vom Arzt } \\
\text { ausgefüllt }\end{array}$ & 68 & 39,5 \\
\hline $\begin{array}{l}\text { Eigener Vordruck: von der } \\
\text { Praxisangestellten ausgefüllt }\end{array}$ & 8 & 4,7 \\
\hline
\end{tabular}


Tabelle 5 Zusammensetzung der Attestanfragen

\begin{tabular}{|c|c|c|c|c|c|}
\hline & $\begin{array}{l}\text { Mittelwert (in } \\
\%)\end{array}$ & $\begin{array}{l}\text { 95\% KI } \\
\text { (Unterer W) }\end{array}$ & $\begin{array}{l}95 \% \text { KI } \\
\text { (Oberer W) }\end{array}$ & Minimum & Maximum \\
\hline $\begin{array}{l}\text { Gesetzliche } \\
\text { Krankenkasse }\end{array}$ & 21,88 & 19,47 & 24,28 & 0 & 75 \\
\hline Versorgungsamt & 15,26 & 13,54 & 16,98 & 0 & 60 \\
\hline Schule & 14,61 & 12,81 & 16,60 & 0 & 62 \\
\hline $\begin{array}{l}\text { Rentenversicherung } \\
\text { und Reha-Anträge }\end{array}$ & 12,93 & 11,76 & 14,11 & 0 & 40 \\
\hline Arbeitgeber & 10,15 & 7,59 & 12,25 & 0 & 75 \\
\hline Private Krankenkasse & 5,43 & 4,71 & 6,16 & 0 & 25 \\
\hline $\begin{array}{l}\text { Andere } \\
\text { Versicherungen (z.B. } \\
\text { Unfalls-, } \\
\text { Berufsunfähigkeits- } \\
\text { versicherung) }\end{array}$ & 5,33 & 4,71 & 5,96 & 0 & 25 \\
\hline Behörden & 5,04 & 4,33 & 5,75 & 0 & 25 \\
\hline Sonstige & 4,43 & 3,79 & 6,12 & 0 & 44 \\
\hline Sport & 2,90 & 2,42 & 3,37 & 0 & 20 \\
\hline $\begin{array}{l}\text { Reiserücktrittsversiche } \\
\text { rung }\end{array}$ & 2,87 & 2,45 & 3,30 & 0 & 20 \\
\hline
\end{tabular}

Tabelle 6.1 Zeitaufwand pro Attest

\begin{tabular}{|c|c|c|c|c|c|c|}
\hline & $\begin{array}{l}\text { Mittelwert } \\
\text { (in Min) }\end{array}$ & $\begin{array}{l}\text { 95\% KI } \\
\text { (Unterer W) }\end{array}$ & $\begin{array}{l}95 \% \text { KI } \\
\text { (Oberer W) }\end{array}$ & Minimum & Maximum & T-Test \\
\hline Untersuchung & & & & & & \begin{tabular}{|l} 
Sig. (2- \\
seitig)
\end{tabular} \\
\hline $\begin{array}{l}\text { Vorgefertigte } \\
\text { Formulare }\end{array}$ & 18,49 & 16,55 & 20,43 & 0 & 60 & 0,000 \\
\hline Freie Form & 26,15 & 23,10 & 29,19 & 1 & 150 & \\
\hline \multicolumn{7}{|l|}{ Gutachterlich } \\
\hline $\begin{array}{l}\text { Vorgefertigte } \\
\text { Formulare }\end{array}$ & 22,66 & 22,66 & 24,41 & 4 & 60 & \multirow[t]{2}{*}{0,000} \\
\hline Freie Form & 31,06 & 31,06 & 33,98 & 5 & 120 & \\
\hline \multicolumn{7}{|l|}{ Kleines Attest } \\
\hline $\begin{array}{l}\text { Vorgefertigte } \\
\text { Formulare }\end{array}$ & 2,59 & 2,59 & 2,94 & 1 & 15 & \multirow[t]{2}{*}{0,000} \\
\hline Freie Form & 4,05 & 4,05 & 4,50 & 1 & 16 & \\
\hline
\end{tabular}


Tabelle 6.2 Wöchentlicher Zeitaufwand

\begin{tabular}{|c|c|c|c|c|c|c|}
\hline & $\begin{array}{l}\text { Mittelwert } \\
\text { (in Min) }\end{array}$ & $\begin{array}{l}\text { 95\% KI } \\
\text { (Unterer W) }\end{array}$ & $\begin{array}{l}95 \% \text { KI } \\
\text { (Oberer W) }\end{array}$ & Minimum & Maximum & T-Test \\
\hline Kleines Attest & & & & & & $\begin{array}{l}\text { Sig. (2- } \\
\text { seitig) }\end{array}$ \\
\hline Freie Form & 46,14 & 29,91 & 61,49 & 2 & 1000 & \multirow[t]{2}{*}{0,000} \\
\hline $\begin{array}{l}\text { Vorgefertigte } \\
\text { Formulare }\end{array}$ & 26,45 & 18,50 & 34,84 & 1 & 500 & \\
\hline \multicolumn{7}{|l|}{ Gutachterlich } \\
\hline Freie Form & 132,08 & 94,16 & 167,12 & 10 & 1700 & \multirow[t]{2}{*}{0,000} \\
\hline $\begin{array}{l}\text { Vorgefertigte } \\
\text { Formulare }\end{array}$ & 92,04 & 64,36 & 120,75 & 5 & 1700 & \\
\hline \multicolumn{7}{|l|}{ Untersuchung } \\
\hline Freie Form & 74,47 & 53,34 & 103,11 & 0 & 1500 & \multirow[t]{2}{*}{0,000} \\
\hline $\begin{array}{l}\text { Vorgefertigte } \\
\text { Formulare }\end{array}$ & 52,73 & 39,25 & 69,10 & 0 & 750 & \\
\hline
\end{tabular}

Tabelle 7.1 Anteil abgerechneter Atteste

\begin{tabular}{|l|l|l|l|l|l|l|}
\hline & \multicolumn{2}{|l|}{$\begin{array}{l}\text { Mittelwert (in } \\
\text { \%) }\end{array}$} & $\begin{array}{l}\text { 95\% KI } \\
\text { (Unterer W) }\end{array}$ & $\begin{array}{l}\text { 95\% KI } \\
\text { (Oberer W) }\end{array}$ & Minimum & Maximum \\
\hline Insgesamt & & 73,73 & 69,66 & 77,80 & 0 & 100 \\
\hline
\end{tabular}

Tabelle 7.2 Konsequente Abrechnung

\begin{tabular}{|l|r|r|}
\hline & Häufigkeit $(\mathbf{n = 1 7 2})$ & Prozent \\
\hline Abrechnung ,immer“ & & \\
\hline Allgemein & 47 & 27,3 \\
\hline Finanzieller Vorteil für Patient & 92 & 53,5 \\
\hline Gebühren & 110 & \\
\hline Nur Abrechnungsziffer & 62 & 64,0 \\
\hline Zusatzgebühr & & 36,0 \\
\hline Zahlungsweise & 103 & 59,9 \\
\hline Barzahlung & 98 & 57 \\
\hline Rechnung & 3 & 1,7 \\
\hline Gar nicht & & \\
\hline
\end{tabular}


Tabelle 8.1 Betrag pro Attest

\begin{tabular}{|l|r|r|r|r|r|}
\hline & Mittelwert (in & $\begin{array}{l}\text { 95\% KI } \\
\text { (Unterer W) }\end{array}$ & $\begin{array}{l}\text { 95\% KI } \\
\text { (Oberer W) }\end{array}$ & Minimum & Maximum \\
\hline Untersuchung & 46,19 & 41,70 & 50,69 & 0 & 200 \\
\hline Gutachterlich & 29,60 & 27,18 & 32,02 & 0 & 100 \\
\hline Kleines Attest & 3,78 & 3,29 & 4,28 & 0 & 15 \\
\hline
\end{tabular}

Tabelle 8.2 Stundenlohn

\begin{tabular}{|c|c|c|c|c|c|c|}
\hline & $\begin{array}{l}\text { Mittelwert } \\
\text { (in €) }\end{array}$ & $\begin{array}{l}\text { 95\% KI } \\
\text { (Unterer W) }\end{array}$ & $\begin{array}{l}95 \% \text { KI } \\
\text { (Oberer W) }\end{array}$ & Minimum & Maximum & T-Test \\
\hline Kleines Attest & & & & & & $\begin{array}{l}\text { Sig. (2- } \\
\text { seitig) }\end{array}$ \\
\hline Freie Form & 83,25 & 64,05 & 102,44 & 0 & 900 & \multirow[t]{2}{*}{0,000} \\
\hline $\begin{array}{l}\text { Vorgefertigte } \\
\text { Formulare }\end{array}$ & 135,99 & 109,30 & 162,68 & 0 & 900 & \\
\hline \multicolumn{7}{|l|}{ Gutachterlich } \\
\hline Freie Form & 73,87 & 63,29 & 84,45 & 0 & 360 & \multirow[t]{2}{*}{0,000} \\
\hline $\begin{array}{l}\text { Vorgefertigte } \\
\text { Formulare }\end{array}$ & 104,15 & 87,22 & 121,09 & 0 & 600 & \\
\hline \multicolumn{7}{|l|}{ Untersuchung } \\
\hline Freie Form & 165,43 & 112,69 & 218,180 & 0 & 3000 & \multirow[t]{2}{*}{0,000} \\
\hline $\begin{array}{l}\text { Vorgefertigte } \\
\text { Formulare }\end{array}$ & 184,43 & 180,69 & 549,55 & 0 & 2250 & \\
\hline
\end{tabular}




\subsection{Gesprächsleitfaden}

\section{Gesprächsleitfaden}

\section{Grundlagen}

- Auf Welche Aspekte achten Sie bei der Ausstellung von Attesten am meisten? (Verantwortung, Vollständigkeit, Datenschutzbestimmungen, Schweigepflicht)

- Bei welchen Punkten sehen Sie Potenzial für Probleme oder Verbesserungsbedarf?

\section{Untersuchungen}

- Haben Sie eine standardisierte Vorgehensweise für verschiedene Atteste?

- Würden Sie standardisierte Untersuchungsprotokolle nutzen?

- Passen Sie die Untersuchungen an den jeweiligen Patienten an?

(Gesundheitszustand, Alter, langjähriger Patient, eigene Einschätzung)

- Überweisen Sie den Patienten wenn nötig für weitere Untersuchungen? (Beispiel Tauchzeugnis: Tauchmedizin, Kardiologie, HNO)

-Wer stellt das Attest dann letztendlich aus?

\section{Abrechnung}

- Welche Atteste rechnen Sie ab?

(Atteste, die die GKV übernimmt / Private Atteste / Versicherungen)

- Stoßen Sie bei Patienten oft auf Unverständnis? Wie gehen Sie damit um?

- Welche Atteste rechnen Sie nicht ab und weshalb?

-Welche GOÄ Ziffern verwenden Sie für welche Atteste? (GOÄ 70, 75, 76) 


\section{Zeitaufwand}

-Wie viel Zeit nehmen Atteste Ihrem Empfinden nach im Alltag ein?

(Wenig, angemessen, übermäßig viel?)

- Überwiegt eine Art von Attesten?

\section{$\underline{5 \text { Attestart }}$}

- Überwiegt eine Art von Attesten in Zahl / Zeitaufwand / Problempotenzial?

(Sport- bzw. Tauchattest, Reiserücktrittsversicherung, Schulattest)

\section{$\underline{6 \text { Vorlagen }}$}

- Verwenden Sie Vorlagen in der Praxis?

- Für welche Atteste haben Sie Vorlagen?

-Wer hat die Vorlagen erstellt?

- Keine Vorlagen: Wären Vorlagen eine Erleichterung?

- Würden Sie standardisierte Vorlagen verwenden?

\section{Verbesserungsmöglichkeiten}

-Wo sehen Sie die größten Kritikpunkte?

-Wo sehen Sie das größte Verbesserungspotenzial?

- Haben Sie konkrete Verbesserungsvorschläge? Was sollte ein Standard Formular unbedingt beinhalten? Was sollte ein Untersuchungsprotokoll unbedingt beinhalten?

-Wir hoffen mit der Umfrage ja auch eine Verbesserung in Ihrem Sinne zu erreichen. Was könnte sich für Sie im Idealfall ändern?

\section{Beispielatteste:}

-Wären Sie bereit, anonymisierte Atteste aus Ihrer Praxis zur Verfügung zu stellen? 


\subsection{Tabellarische Auflistung der Ergebnisse des Gesprächsleitfadens}

Tabelle 9: Übersicht der Angaben zu aktuellen Vorgehensweisen

\begin{tabular}{|c|c|c|c|c|c|c|c|}
\hline Vorlagen & $\begin{array}{l}\text { Keine } \\
\text { Vorlagen }\end{array}$ & $\begin{array}{l}\text { Vorlagen für } \\
\text { kurze Atteste }\end{array}$ & $\begin{array}{l}\text { Vorlagen für } \\
\text { umfangreiche } \\
\text { Atteste }\end{array}$ & \multicolumn{2}{|c|}{$\begin{array}{l}\text { Vorlagen } \\
\text { insgesamt }\end{array}$} & \multicolumn{2}{|l|}{ Fallzahl n } \\
\hline $\begin{array}{l}\text { Eigene } \\
\text { Vorlagen } \\
\text { vorhanden }\end{array}$ & 7 & 14 & 10 & & 24 & & 31 \\
\hline $\begin{array}{l}\text { Standard- } \\
\text { Vorlagen } \\
\text { erwünscht }\end{array}$ & 7 & 14 & 6 & & 27 & & \\
\hline \multirow[t]{2}{*}{ Abrechnung } & \multicolumn{2}{|c|}{$\begin{array}{l}\text { Ausnahme bei sozialer } \\
\text { Indikation }\end{array}$} & \multicolumn{2}{|c|}{ Keine Ausnahmen } & \multicolumn{2}{|c|}{ Fallzahl n } & \\
\hline & & 28 & & 3 & & & 31 \\
\hline \multirow[t]{2}{*}{ Untersuchungen } & \multicolumn{2}{|c|}{$\begin{array}{l}\text { An Patienten } \\
\text { angepasst }\end{array}$} & \multicolumn{2}{|l|}{$\begin{array}{l}\text { Unabhängig vom } \\
\text { Patienten }\end{array}$} & \multicolumn{2}{|c|}{ Fallzahl n } & \\
\hline & & 27 & & 4 & & & 31 \\
\hline \multirow[t]{2}{*}{$\begin{array}{l}\text { Zuständigkeit für } \\
\text { Atteste }\end{array}$} & \multicolumn{2}{|c|}{ Hausarzt } & \multicolumn{2}{|l|}{ Anderer Facharzt } & \multicolumn{2}{|c|}{ Fallzahl n } & \\
\hline & & 31 & & 0 & & & 31 \\
\hline
\end{tabular}




\subsection{Tabellarische Auflistung der Beispielatteste}

Tabelle 10: Verteilung der Attestbesipiele auf verschiedene Kategorien

\begin{tabular}{|c|c|c|}
\hline Attest / Anfrage & Vorlage & Freie Form \\
\hline Befundbericht & 8 & \\
\hline Gesundheitszeugnis & 8 & \\
\hline Schulunfähigkeit & 5 & \\
\hline Arbeitsunfähigkeitsbescheinigung & 4 & \\
\hline Berufseignung & 4 & \\
\hline $\begin{array}{l}\text { Kostenübernahme: Antrag für } \\
\text { Versicherungen }\end{array}$ & 4 & \\
\hline $\begin{array}{l}\text { Rehabilitationsmaßnahmen: } \\
\text { Antrag für Versicherungen }\end{array}$ & 4 & \\
\hline Sportunfähigkeit & 3 & \\
\hline $\begin{array}{l}\text { Bescheinigung zur } \\
\text { Medikamentenmitnahme auf } \\
\text { Reisen }\end{array}$ & 3 & \\
\hline Minderung der Erwerbsfähigkeit & 2 & \\
\hline Sporttauglichkeitsattest & 2 & \\
\hline Prüfungsunfähigkeit & 1 & \\
\hline $\begin{array}{l}\text { Tauglichkeit für Führerschein } \\
\text { (LKW) }\end{array}$ & 1 & \\
\hline Zulassung zur Blutspende & 1 & \\
\hline Sozialamt & 1 & 5 \\
\hline
\end{tabular}




\subsection{Zusammenfassung der wichtigsten Ergebnisse und Handlungsempfehlungen}

\section{Effizienzsteigerung durch Vorlagen}

\section{Zeitaufwand pro Attest}

- 97\% der Ärzte bestätigen, dass die Standardisierung Zeit sparen könnte.

- Um dies zu überprüfen, wurde der Zeitaufwand pro Attest für die Gruppen Kleines Attest, Attest mit gutachterlichem Charakter und Attest mit Untersuchungen jeweils mit Vorlage und bei freier Formulierung verglichen.

- Bei Attesten mit Untersuchungen oder gutachterlichem Charakter besteht deutliches Potential zur Zeitersparnis: Atteste mit Vorlage dauern hier 7 respektive 8 Minuten weniger. Der ohnehin geringe Aufwand bei kleineren Attesten lässt sich um etwa eine Minute reduzieren.

\section{Wöchentlicher Zeitaufwand}

- Im Alltag ist vor allem der Vergleich des gesamten Aufwands von Interesse, der sich aus dem Zeitaufwand pro Attest und der Zahl der angefragten Atteste pro Woche ergibt.

- Im Mittel könnte der Aufwand bei Attesten mit gutachterlichem Charakter um 40 Minuten pro Woche verringert werden, bei Attesten mit Untersuchungen um 21 Minuten, bei kleinen Attesten um 20. Zusammengenommen ergibt sich daraus eine Ersparnis von über einer Stunde pro Woche.

\section{Vergütung}

- Atteste mit Untersuchungen werden durchschnittlich mit $46 €$ vergütet, Atteste mit gutachterlichem Charakter mit $30 €$, Atteste mit geringem Aufwand mit $4 €$.

- Um das Verhältnis von Aufwand und Entschädigung beim Vorgehen mit Vorlage und ohne Vorlage vergleichbar zu machen, wurde ein fiktiver „Stundenlohn“ berechnet:

- Bei Attesten mit geringem Aufwand ließe sich der Stundenlohn durch die Verwendung von Vorlagen von $83 €$ auf $136 €$, also um $60 \%$, steigern.

- Bei Attesten mit gutachterlichem Charakter ließe sich der Stundenlohn von 74€ auf $104 €$, also um $41 \%$, steigern.

- Bei Attesten mit Untersuchungen ließe sich der Stundenlohn von $165 €$ auf $184 €$, also um $11 \%$, steigern. 


\section{Vorgehensweisen bei der Attestausstellung}

- Untersuchungs-Checklisten: Ein strukturierter Untersuchungskatalog verhindert, dass Untersuchungen vergessen werden. Gleichzeitig können unnötige Untersuchungen bewusst weggelassen werden.

- Vordrucke: Für kleine Atteste wie Krankschreibungen für die Schule, die nicht individualisiert werden müssen, eignen sich einfache Vordrucke am besten.

- Textbausteine: Für Stellungnahmen, deren komplexen Inhalt man nicht in eine starre Vorlage zwängen kann (zum Beispiel bei Anfragen vom Sozialamt), eignen sich Textbausteine, mit denen einzelne Formulierungen und Argumente wiederverwendet werden können.

- Praxissoftware: Durch die Verbindung mit einem ein Textverarbeitungssystem lassen sich Patientendetails und relevante Diagnosen automatisch aus der Patientenkartei in der Praxissoftware übernehmen. Einige Softwares bieten zusätzliche Hilfestellungen für Attestvorlagen und Textbausteine.

- Praxisassistenz: Ein gut durchdachtes System ermöglicht es, die Praxisassistenz Vordrucke ausfüllen und Textbausteine zusammenstellen zu lassen, sodass der Arzt den Inhalt lediglich bestätigten muss.

- Patienten: Bei unklaren Anfragen sollte der Patient eine Vorlage oder genauere Angaben einholen. Geeignete Patienten können den Inhalt selbst formulieren, nach sorgfältiger Prüfung kann dieser vom Arzt bestätigt werden.

\section{Umgang mit Problempotential}

\section{Datenschutz und Schweigepflicht}

- Wenn der Patient das Attest selbst beantragt oder weiterleitet, ist die Schweigepflichtsentbindung impliziert.

- Eine Anfrage ohne Einbindung des Patienten sollte mit einem vorgefertigten Hinweis auf die Notwendigkeit einer Schweigepflichtsentbindung beantwortet werden.

- Wird eine explizite medizinische Begründung gefordert, sind nach Möglichkeit nur Symptome zu benennen. Ist die Nennung einer Diagnose unumgänglich, ist sie mit Vorsicht zu wählen, um dem Patienten keine unangemessenen Nachteile zu bereiten.

- Weichen Kassenanfragen von der vereinbarten Form ab (Ergänzungen, Änderungen), ist die Rechtmäßigkeit dieser in Frage zu stellen und mit einem Vordruck darauf hinzuweisen, dass Informationen zu Diagnose und Behandlung beim MDK einzuholen sind. 


\section{Verantwortung}

- Durch genaue Angaben lässt sich die Verantwortung für den Inhalt des Attests eingrenzen.

- Weit gefasste Definitionen wie „frei von ansteckenden Krankheiten“ lassen sich durch die Entscheidungsgrundlage (z.B. anamnestisch) und den Zusatz zum Zeitpunkt der Untersuchung relativieren.

\section{Abrechnung}

- Insgesamt werden knappe 75\% der Atteste abgerechnet, da die geringe Vergütung oft nicht im Verhältnis zum Aufwand der Rechtfertigung und Abrechnung steht.

- Die Berufsordnung für Ärzte schreibt die Liquidierung von Attesten vor. Durch eine konsequente und konsistente Abrechnungspraxis lässt sich die Patienteneinsicht fördern.

- Ist die Ausstellung des Attests der alleinige Anlass für Beratung und Untersuchung, können diese privat berechnet werden.

- Für einen regulären Arztbesuch kommt die Krankenkasse auf. Wenn ein Attest als „Nebenprodukt“ ausgestellt wird, hat der Patient nur die Kosten dafür zu tragen.

- Werden nur Kopien der Befunde oder Behandlungsdokumentation erstellt, kann keine Gebühr nach GOÄ erhoben werden.

- Patienten sind vorab über die Regelungen und Kosten aufzuklären. Bundes- und Landesärztekammern stellen geeignete Poster für das Wartezimmer zur Verfügung.

- Versicherungen bieten regelmäßig unzureichende Vergütung für das Ausstellen von Befundberichten und Gutachten. Der Arzt muss dieses Angebot nicht annehmen, da ihm die gesetzliche Vergütung zusteht.

- Anfragen von Krankenkasse werden häufig nicht korrekt honoriert. Werden Muster-Vordrucke der Krankenkassen mit Fragen ergänzt oder geändert, kann der Arzt eine Kostenübernahmeerklärung einholen und privat abrechnen, wenn eine Einverständniserklärung des Patienten vorliegt. Die KVB stellt eine Musterantwort für diese Anfragen bereit.

- Die Bitte um eine Kostenzusage bei anderen Institutionen (z.B. Schulen) hilft, Attestanfragen zu verringern. Dafür empfiehlt sich ein Vordruck, auf dem zusätzlich darauf hingewiesen wird, dass der Arzt oft ebenso wenig wie Lehrer oder Eltern im Stande ist, die Schuluntauglichkeit zu verifizieren. 


\subsection{Ethikvotum}
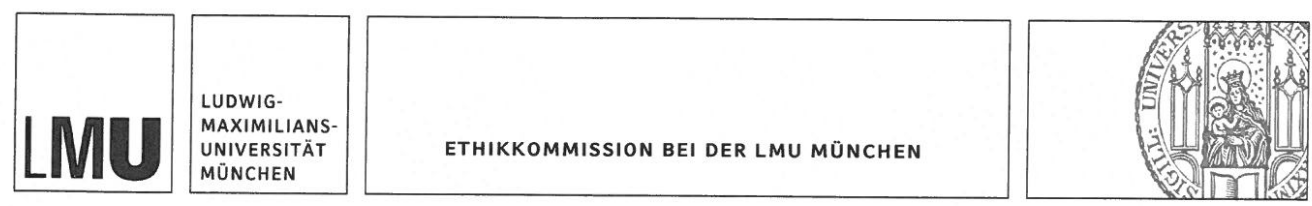

Ethikkommission · Pettenkoferstr. $8 \cdot 80336$ München

Vorsitzender:

Prof. Dr. W. Eisenmenger Telefon+49(0)89440055191

Prof. Dr. Jörg Schelling

Institut für Allgemeinmedizin

Telefax+49 (0)89440055192

Pettenkoferstr. 8a

Ethikkommission@

med.uni-muenchen.de

80336 München

med.uni-muenchen.de
www.ethikkommission.med.uni -muenchen.de

Anschrift:

Pettenkoferstr. 8a

D-80336 München

29.09.2015 Hb

Unser Zeichen: 412-15 (bitte bei Schriftwechsel angeben)

\section{Beratung nach Fakultätsrecht}

Studientitel: Medizinische Atteste und Untersuchungen in der allgemeinärztlichen Praxis - eine Übersicht und empirische Befragung zu Indikationen, Hindernissen und Relevanz im hausärztlichen Alltag

Antragsteller: Prof. Dr. Jörg Schelling, Institut für Allgemeinmedizin , Pettenkoferstr. 8a, 80336 München

Sehr geehrter Herr Prof. Schelling,

besten Dank für Ihr Schreiben vom 25.09.2015 mit der Beantwortung unserer Fragen bzw.

Erfüllung der Auflagen und den noch ausstehenden bzw. überarbeiteten Unterlagen.

Die Ethikkommission (EK) kann Ihrer Studie nun die ethisch-rechtliche Unbedenklichkeit zuerkennen.

Vorsorglich möchte ich darauf hinweisen, dass auch bei einer positiven Beurteilung des Vorhabens durch die EK die ärztliche und juristische Verantwortung für die Durchführung des Projektes uneingeschränkt bei Ihnen und Ihren Mitarbeitern verbleibt.

Allgemeine Hinweise:

- Änderungen im Verlauf der Studie sind der EK zur erneuten Prüfung vorzulegen.

- Schwerwiegende unerwartete Ereignisse im Rahmen der Studie sind der EK mitzuteilen.

- Das Ende der Studie ist anzuzeigen und das Ergebnis vorzulegen.

- Die ärztliche und juristische Verantwortung bei der Durchführung der Studie verbleibt uneingeschränkt bei Ihnen und Ihren Mitarbeitern.

Für Ihre Studie wünsche ich Ihnen viel Erfolg.

Mit freundlichen Grüßen

Prof. Dr.W. Eisenmenger

Vorsitzender der Ethikkommission

Mitglieder der Kommission:

Prof. Dr. W. Eisenmenger (Vorsitzender), Prof. Dr. E. Held (stellv. Vorsitzender), Prof Dr. C. Bausewein, PD Dr. Th. Beinert, Prof. Dr. B. Emmerich, Prof. Dr. H. U. Gallwas, Dr. B. Henrikus, Dr. V. Mönch, Prof. Dr. D. Nowak, Prof. Dr. R. Penning, Prof. Dr. K. Hahn, Prof. Dr. K 


\subsection{Danksagung}

Mein herzlicher Dank gilt allen, die zum Gelingen dieser Arbeit beigetragen und mich großzügig unterstützt haben.

Ganz besonders möchte ich mich bei Prof. Jörg Schelling bedanken, der mich bei allen wichtigen Schritten von der Auswahl des Dissertationsthemas bis zur Interpretation der Ergebnisse konstruktiv, kreativ und kompetent beraten hat.

Frau Dr. Linda Sanftenberg bin ich für die zuverlässige und intensive Betreuung sehr dankbar.

Auch Herrn Dr. Ulf Schelling und Herrn Dr. Roland Vogl möchte ich für ihren Rat danken.

Frau Wiltrud Garcia-Aznaran im Sekretariat des Instituts für Allgemeinmedizin danke ich für die organisatorische Unterstützung.

Natürlich möchte ich mich auch herzlich bei allen Ärzten bedanken, die sich Zeit für die Umfrage und das Gespräch genommen haben. Für Veröffentlichung der Umfrage danke ich den Mitarbeitern der Fachzeitschriften und Online Portale.

Für den Rat zu Fragebogen und Gesprächsleitfaden danke ich Frau Dr. Katja Kühlmayer am Institut für Ethik, Geschichte und Theorie der Medizin, Herrn Dipl.-Ing. Matthias Holzer und Herrn Dr. Jan Kiesewetter am Institut für Didaktik und Ausbildungsforschung in der Medizin. Für die Beratung zur statistischen Auswertung danke ich Frau Birgit Waschulzik am IMSE und Herrn Roman Hornung am IBE.

Meiner Mentorin Dr. Ebba Beller danke ich herzlich für ihre zuverlässige Unterstützung bei allen Fragen zum Studium. 
8.10 Eidesstattliche Versicherung

\section{Eidesstattliche Versicherung}

Ertl, Livia

Name, Vorname

Ich erkläre hiermit an Eides statt,

dass ich die vorliegende Dissertation mit dem Thema

selbständig verfasst, mich außer der angegebenen keiner weiteren Hilfsmittel bedient und alle Erkenntnisse, die aus dem Schrifttum ganz oder annähernd übernommen sind, als solche kenntlich gemacht und nach ihrer Herkunft unter Bezeichnung der Fundstelle einzeln nachgewiesen habe.

Ich erkläre des Weiteren, dass die hier vorgelegte Dissertation nicht in gleicher oder in ähnlicher Form bei einer anderen Stelle zur Erlangung eines akademischen Grades eingereicht wurde.

München, 21.04.16

Livia Ertl

Ort, Datum

Unterschrift Doktorandin/Doktorand 